\title{
Análise do Conhecimento Extraído de Classificadores Simbólicos Utilizando Medidas de Avaliação e de Interessabilidade ${ }^{1}$
}

\section{Alan Keller Gomes}

\author{
Orientação: \\ Profa. Dra. Maria Carolina Monard
}

Dissertação apresentada ao Instituto de Ciências Matemáticas e de Computação da Universidade de São Paulo - ICMC/USP - como parte dos requisitos necessários à obtenção do título de Mestre em Ciências de Computação e Matemática Computacional. Aprovada pela banca examinadora no dia 02/05/2002.

\section{USP - São Carlos}

\footnotetext{
${ }^{1}$ Trabalho realizado com o apoio do CNPq - http://www.cnpq.br
} 
Este Documento foi preparado com o formatador de textos $\mathrm{LAT}_{\mathrm{E} X} \mathrm{X}$. O sistema de citações de referências bibliográficas utiliza o padrão Apalike do sistema $\mathrm{BIBT}_{\mathrm{E}} \mathrm{X}$.

(c) Copyright 2001 por Alan Keller Gomes Todos os Direitos Reservados 


\section{Dedicatória}

Ao meu grande amigo e ex-professor, Paulo André Sant'Anna Perez. 


\section{Agradecimentos}

A Deus, por cada conquista que já tive e pelas que ainda virão.

À Profa. Maria Carolina Monard, pela paciência, dedicação, confiança, compreensão e sinceridade ao apontar o caminho a ser percorrido. Meus mais profundos e sinceros agradecimentos pela oportunidade de crescer.

Ao meu pai Divino Gomes e minha mãe Francisca Narciso que se fizeram presentes, mesmo estando ausentes. Pelos ensinamentos que foram e sempre serão pilares a sustentarme.

Ao Paulo André, meu grande amigo, ex-professor e ex-orientador de iniciação científica, a quem devo todo meu respeito, minha consideração e gratidão, pela confiança, pelos bons conselhos e pela amizade sincera.

Ao Carlos Eduardo, pela compreensão e pelo companheirismo.

Aos meus amigos Jorge e Marcos Geromini, pelo apoio, dicas, correções e também ao Gláucio e Edvaldo pela amizade e ótima companhia.

À minha amiga Rachel, pela força e hospitalidade.

Ao cara mais chato do mundo, Leandro Henrique, por ter tido a paciência de morar e suportar conviver comigo nesses dois últimos anos.

À Flávia Cristina, mamãe dedicada e mulher batalhadora, por todos maus e bons bocados que passamos juntos.

Aos amigos e colegas do LABIC (Claudinha, Gustavo e Cláudia Milaré, Valéria-VTA, Patrícia, Valmir, José Flávio, Cris, Jaque, Walter e, em especial, ao Ronaldo Prati), pela força, pela ajuda e boa-vontade de sempre trocar informações. Aos amigos do NILC (Juliana, Andréia, Valéria, Ricardo, Helena, Ariane) e do saudoso GTI (Leandro, Antônio, Pedro, Giovanna, Rodrigo, Félix, Isaac e Marcos Vinícius). E também aos sempre fiéis Fabiano e Adriano.

Aos funcionários do ICMC (Marília, Beth, Laura, Fernadinha e Ana Paula).

Ao $\mathrm{CNPq}$ pelo apoio financeiro. 


\section{Resumo}

Com o avanço da tecnologia, grandes volumes de dados estão sendo coletados e acumulados numa velocidade espantosa. Data Mining constitui um campo de pesquisa recente em Inteligência Artificial, cujo objetivo é extrair conhecimento de grandes bases de dados. Um dos tópicos tratados em Data Mining para extrair conhecimento é o uso de algoritmos de Aprendizado de Máquina em grandes volumes de dados.

Alguns algoritmos de Aprendizado de Máquina são capazes de criar generalizações, ou descrever conceitos, a partir de um conjunto de dados previamente rotulados. Esses algoritmos são conhecidos como indutores e são capazes de induzir uma hipótese (ou classificador). Um classificador pode estar descrito sob uma forma simbólica explícita, e assim, apresentar uma explicação do conceito aprendido de forma inteligível ao ser humano. Uma dessas formas de representação simbólica explícita são as regras de conhecimento.

Especialmente em Data Mining, o volume de regras de conhecimento que descrevem um classificador simbólico pode ser muito grande. Isso dificulta muito a análise de regras individuais ou de um grupo de regras por parte do usuário desse conhecimento. No intuito de propor uma solução para essa dificuldade, a análise automática de regras, utilizando medidas de avaliação e de interessabilidade, destaca-se como uma das fontes de resultados positivos da aplicação do Aprendizado de Máquina na área de Data Mining.

Neste trabalho é apresentado o $\mathcal{R}_{\text {ule }} \mathcal{S}_{\text {ystem }}$, um sistema computacional protótipo que implementa funcionalidades voltadas para Aprendizado de Máquina e Data Mining. Uma dessas funcionalidades, implementadas no $\mathcal{R}_{\text {ule }} \mathcal{S}_{\text {ystem }}$, refere-se à análise automática de regras. O Módulo de Análise de regras, proposto neste trabalho, implementa diversas medidas de avaliação e de interessabilidade de regras, permitindo assim realizar uma análise tanto quantitativa quanto qualitativa das regras que constituem a(s) hipótese(s) induzida(s) por algoritmos de Aprendizado de Maquina simbólico. 


\section{Abstract}

Now a days huge amount of data is gathered and accumulated very fast in large databases. However, there is a growing gap between the generation of data and people understanding of it. The science of extracting useful information from large data sets or databases is known as Data Mining, a new discipline lying at the intersection of several areas, among them, Artificial Intelligence and Machine Learning (ML).

In this work we concentrate on the use of symbolic supervised ML algorithms as part of the Data Mining process. The term symbolic indicates that the classifiers induced by these ML algorithms should be human readable, such as if-then rules, and comprehensible. An important issue is related to the quality and interestingness of the rules induced by these algorithms. But, especially in Data Mining, the amount of knowledge-rules induced by ML algorithms may be very large, making difficult the selection of good rules by humans.

In this work we present and discuss several rule measures that can provide support for ranking and selecting good rules. We also present the $\mathcal{R}_{\text {ule }} \mathcal{S}_{\text {ystem }}$, a prototype computational system that implements several functions concerned with the use of Machine Learning in the Data Miming process. The $\mathcal{R}_{\text {ule }} \mathcal{S}_{\text {ystem }}$ uses as input a common file format for data sets, features description and if-then knowledge rules. One of the functions in the $\mathcal{R}_{\text {ule }} \mathcal{S}_{\text {ystem }}$, implemented through the Rule Analysis Module, is related to rule measures.

The Rule Analysis Module proposed in this work implements several rule evaluation and interestingness measures. Through this module the $\mathcal{R}_{\text {ule }} \mathcal{S}_{\text {ystem }}$ is able to do a qualitative as well as a quantitative analysis of the set of rules induced by one or more symbolic Machine Learning algorithms. Furthermore, as the system has been implemented in Pro$\log$ it is query-centered, allowing the user to specify any constraints on the result of a query in order to narrow the search for good rules. 


\section{Índice}

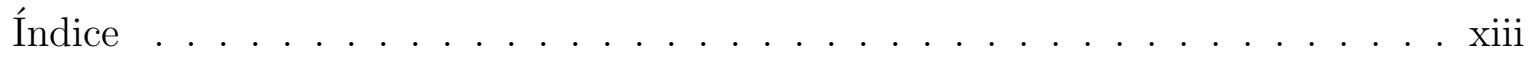

Lista de Figuras . . . . . . . . . . . . . . . . . . xvii

Lista de Tabelas . . . . . . . . . . . . . . . . . xix

Lista de Algoritmos . . . . . . . . . . . . . . . . . . xxi

Lista de Abreviaturas . . . . . . . . . . . . . . . . . xxiii

1 Introdução 1

2 Aprendizado de Máquina 5

2.1 Considerações Iniciais . . . . . . . . . . . . . . . . . . 5

2.2 Aprendizado e Aquisição de Conhecimento . . . . . . . . . . . 5

2.3 Características Gerais dos Sistemas de Aprendizado de Máquina . . . . . . 7

2.3 .1 Modos de Aprendizado . . . . . . . . . . . . . . . . . . 8

2.3.2 Paradigmas de Aprendizado . . . . . . . . . . . . . . 8

2.3 .3 Linguagens de Descrição . . . . . . . . . . . . . . . . . . . . . . 12

2.3.4 Aprendizado Incremental e Não-Incremental . . . . . . . . . . . . 13

2.4 Sistemas de Aprendizado de Máquina Indutivo . . . . . . . . . . . . . . 14

2.4.1 Exemplos no Formato Atributo-Valor . . . . . . . . . . . . . . 14

2.4 .2 Classificadores Simbólicos . . . . . . . . . . . . . . . 15

2.5 Considerações Finais . . . . . . . . . . . . . . . . . 16

3 Medidas de Avaliação de Regras $\quad 19$

3.1 Considerações Iniciais . . . . . . . . . . . . . . . . . . . . . . . . . . . 19

3.2 Notação e Terminologia . . . . . . . . . . . . . . . . . . . . . . . . 20

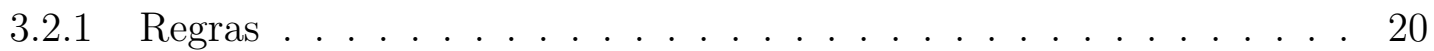

3.2 .2 Matriz de Confusão . . . . . . . . . . . . . . . . . . . . . . 21

3.2 .3 Tabela de Contingência . . . . . . . . . . . . . . . . . . . . . 22

3.3 Medidas de Avaliação de Regras . . . . . . . . . . . . . . . . . . . . . 24

3.3 .1 Um Exemplo para Avaliação . . . . . . . . . . . . . . . . . . 24

3.3.2 Medidas Genéricas de Regras . . . . . . . . . . . . . . . . . 24

3.3.3 Medidas Relativas de Avaliação de Regras . . . . . . . . . . . . . . 33

3.3.4 Medidas Relativas de Regras com Peso . . . . . . . . . . . . 37

3.4 Considerações Finais . . . . . . . . . . . . . . . . . . . . . 40 
4 Interessabilidade de Regras $\quad 43$

4.1 Do termo Interessabilidade . . . . . . . . . . . . . . . . . . . . . . . . 43

4.2 Considerações Iniciais . . . . . . . . . . . . . . . . . . . . . . . . 44

4.3 Aspectos de Interessabilidade de Regras . . . . . . . . . . . . . . . . . . . . 44

4.3.1 Medidas Subjetivas de Interessabilidade . . . . . . . . . . . . . . . . 45

4.3.2 Medidas Objetivas de Interessabilidade . . . . . . . . . . . . 46

4.4 Princípios para Interessabilidade . . . . . . . . . . . . . . . . . . 46

4.5 Algumas Medidas Objetivas de Interessabilidade de Regras . . . . . . . . . 47

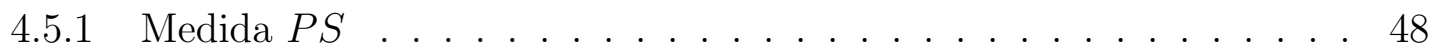

4.5.2 Grau de Surpresa de Pequenos Disjuntos . . . . . . . . . . . . . . 48

4.5.3 Grau de Surpresa dos Atributos Individuais da Regra . . . . . . . . 51

4.6 Considerações Finais . . . . . . . . . . . . . . . . . . 54

5 O Sistema Protótipo $\mathcal{R}_{\text {ule }} \mathcal{S}_{\text {ystem }} \quad 57$

5.1 Considerações Iniciais . . . . . . . . . . . . . . . . . . . . 57

5.2 Visão Geral do $\mathcal{R}_{\text {ule }} \mathcal{S}_{y s t e m} \ldots \ldots \ldots \ldots \ldots \ldots$. . . . . . . . . . . . . . . . . . . . . . . 58

5.3 Os Dados de Entrada do $\mathcal{R}_{u l e} \mathcal{S}_{y s t e m} \ldots \ldots \ldots$. . . . . . . . . . 59

5.3.1 O Formato Padrão de Exemplos e Regras do Projeto Discover . . 59

5.3.2 Sintaxe Padrão Prolog de Exemplos . . . . . . . . . . . . . . . . . . 61

5.3.3 Sintaxe Padrão Prolog de Regras . . . . . . . . . . . . . . . . . 63

5.4 Arquitetura do $\mathcal{R}_{\text {ule }} \mathcal{S}_{y s t e m} \ldots \ldots \ldots \ldots \ldots$. . . . . . . . . . 69

5.5 Metodologia para Documentação do $\mathcal{R}_{\text {ule }} \mathcal{S}_{\text {ystem }} \ldots \ldots \ldots$. . . . . . . . 70

5.5.1 Componentes do Diagrama de Fluxo de Execução de Procedimentos 71

5.5.2 Descrição do Fluxo de Execução do $\mathcal{R}_{\text {ule }} \mathcal{S}_{\text {ystem }}$. . . . . . . . . . . . . 77

5.6 Considerações Finais ． . . . . . . . . . . . . . . . . . . . . 79

6 O Módulo de Análise de Regras do $\mathcal{R}_{\text {ule }} \mathcal{S}_{\text {ystem }} \quad 81$

6.1 Considerações Iniciais . . . . . . . . . . . . . . . . . . . . . . . . 81

6.2 Os Procedimentos Implementados no MAR . . . . . . . . . . . . . . . . . 81

6.3 Análise Qualitativa de Regras . . . . . . . . . . . . . . . . . . 82

6.4 Análise Quantitativa de Regras . . . . . . . . . . . . . . . . . . 88

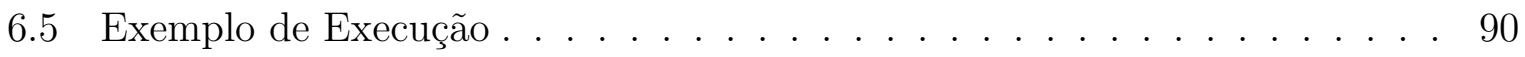

6.6 Considerações Finais . . . . . . . . . . . . . . . . . . . . . . . . . 92

7 Estudo de Caso Utilizando o Módulo de Análise de Regras do $\mathcal{R}_{\text {ule }} \mathcal{S}_{\text {ystem }} 95$

7.1 Considerações Iniciais . . . . . . . . . . . . . . . . . . . . . . . . 95

7.2 Processamento de Sêmen Diagnóstico . . . . . . . . . . . . . . . . . 95

7.3 Etapas do Estudo de Caso . . . . . . . . . . . . . . . . . . . . . . . . . . . 98

7.3.1 Descrição e Preparação do Conjunto de Dados . . . . . . . . . . . . 99

7.3.2 Indução e Preparação dos Conjuntos de Regras . . . . . . . . . . . 99

7.3.3 Análise Qualitativa dos Conjuntos de Regras . . . . . . . . . . . . . 101

7.3.4 Análise Quantitativa dos Conjuntos de Regras . . . . . . . . . . . . 102

7.4 Discussão dos Resultados Obtidos . . . . . . . . . . . . . . . 106

7.4.1 Da Análise Qualitativa . . . . . . . . . . . . . 106 
7.4 .2 Da Análise Quantitativa . . . . . . . . . . . . . . . . . . . . . . 113

7.5 Considerações Finais . . . . . . . . . . . . . . . . . . . . . 114

8 Conclusão $\quad 117$

$\begin{array}{ll}\text { Referências Bibliográficas } & 121\end{array}$ 


\section{Lista de Figuras}

$2.1 \mathbf{h}$ vista como caixa $\operatorname{preta} \ldots \ldots \ldots \ldots \ldots \ldots \ldots$

$2.2 \mathbf{h}$ vista como conjunto de regras . . . . . . . . . . . . . . 16

5.1 Conversores do Formato Padrão $\mathcal{P B M}$ para Sintaxe Padrão Prolog . . . . 60

5.2 Gramática da Sintaxe Padrão Prolog para Exemplos . . . . . . . . . . . . . 62

5.3 Representação Prolog do Conjunto de Exemplos de Teste Voyage . . . . . . 63

5.4 Gramática da Sintaxe Padrão Prolog para Regras . . . . . . . . . . . . . 64

5.5 Exemplo de um Arquivo de Regras no Formato $\mathcal{P B M}$ Estendido . . . . . . 65

5.6 Exemplo de um Arquivo de Regras na Sintaxe Padrão Prolog . . . . . . . . 66

5.7 Arquitetura do $\mathcal{R}_{\text {ule }} \mathcal{S}_{\text {ystem }} \ldots \ldots \ldots \ldots \ldots \ldots$. . . . . . . . . 70

5.8 Diagrama de Fluxo de Execução de Procedimentos do $\mathcal{R}_{\text {ule }} \mathcal{S}_{\text {ystem }}$. . . . . . 72

5.9 Exemplo de Representação de um Procedimento . . . . . . . . . . . . . 73

5.10 Exemplo de Representação de um Conjunto de Condições . . . . . . . . . . 73

5.11 Exemplo de Representação de um Subconjunto de Condições . . . . . . . . 74

5.12 Tipos de Barramento . . . . . . . . . . . . . . . . . . . 76

6.1 Regras Interclass - Conjunto de Subconjuntos de Regras . . . . . . . . . 85

6.2 Arquivo de Exemplos . . . . . . . . . . . . . . . . . . . . . . 90

6.3 Arquivo de Regras Interclass Induzidas por $\mathcal{C} 4.5$ rules . . . . . . . . . . . 91

6.4 Regras na Base de Fatos após a Execução do Procedimento evaluateAlıSet0fRuleFrequency/1 . . . . . . . . . . . . . . . . . . . . 91 91

6.5 Surpresa dos Atributos Individuais das Regras . . . . . . . . . . . . 92

6.6 Medidas Qualitativas das Regras _. . . . . . . . . . . . . . . 93

6.7 Surpresa dos Pequenos Disjuntos das Regras . . . . . . . . . . . . . . 94

7.1 Surpresa dos Pequenos Disjuntos de $\mathbf{h}_{1} \ldots \ldots \ldots \ldots$. . . . . . . 105

7.2 Surpresa dos Pequenos Disjuntos de $\mathbf{h}_{2} \ldots \ldots \ldots \ldots$. . . . . . . . 106

7.3 Surpresa dos Atributos Individuais Discretos das Regras Selecionadas de $\mathbf{h}_{1} 106$

7.4 Surpresa dos Atributos Individuais Discretos das Regras Selecionadas de $\mathbf{h}_{2} 107$

7.5 As "Melhores" Regras de $\mathbf{h}_{1} \ldots \ldots$. . . . . . . . . . . . . . . . . . . . 109

7.6 As "Melhores" Regras de $\mathbf{h}_{2} \quad \ldots \ldots \ldots$ 


\section{Lista de Tabelas}

2.1 Características Gerais de Sistemas de Aprendizado de Máquina. . . . . . . 7

2.2 Conjunto de Exemplos Rotulados no Formato Atributo-Valor . . . . . . . 14

3.1 Matriz de Confusão para Problemas de Classificação Binária . . . . . . . . 22

3.2 Tabela de Contingência para uma Regra . . . . . . . . . . . . . . . 23

3.3 Conjunto de Exemplos de Treinamento voyage . . . . . . . . . . . . . . . 25

3.4 Regras Induzidas a partir do Conjunto de Exemplos de Treinamento voyage 25

3.5 Conjunto de Exemplos de Teste voyage . . . . . . . . . . . . . . . . . 26

3.6 Regras Selecionadas e suas Tabelas de Contingência . . . . . . . . . . . 26

3.7 Matriz de Confusão . . . . . . . . . . . . . . . . . . . . . . . . . 28

3.8 Valores das Medidas Genéricas de Avaliação das Regras $R_{1}, R_{2}$ e $R_{3}$. . . . 34

3.9 Valores das Medidas Relativas de Avaliação das Regras $R_{1}, R_{2}$ e $R_{3}$. . . 40

5.1 Valores Nominais de Operadores . . . . . . . . . . . . . . . . . . 69

5.2 Procedimentos Principais do $\mathcal{R}_{\text {ule }} \mathcal{S}_{\text {ystem }} \ldots \ldots \ldots \ldots$. . . . . . . . . 69

5.3 Condições Pertencentes ao Conjunto de Condições E . . . . . . . . . . . . 73

5.4 Condições Pertencentes ao Conjunto de Condições R . . . . . . . . . . . . 74

5.5 Condições 1, 2, 3, 4 e $5 \ldots \ldots \ldots \ldots$

6.1 Identificação das Medidas de Avaliação de Cada Regra . . . . . . . . . . 88

7.1 Sumário das Características do Conjunto de Dados Proc-a-gmg-d . . . . . 99

7.2 Descrição dos Atributos do Conjunto de Dados Proc-a-gmg-d . . . . . . . . . 100

7.3 Informações sobre os Conjuntos de Regras Induzidas . . . . . . . . . . . . 101

7.4 Valores das Medidas Genéricas para as Regras de $\mathbf{h}_{1} \ldots \ldots$. . . . . . . 103

7.5 Valores das Medidas Genéricas para as Regras de $\mathbf{h}_{2}$. . . . . . . . . . 103

7.6 Valores das Medidas Relativas, com e sem Peso, para as Regras de $\mathbf{h}_{1}$. . . 104

7.7 Valores das Medidas Relativas, com e sem Peso, para as Regras de $\mathbf{h}_{2}$. . . 105

7.8 Regras de $\mathbf{h}_{1}$ Ordenadas pelos Valores da Medida de Avaliação . . . . . . . 108

7.9 Regras de $\mathbf{h}_{2}$ Ordenadas pelos Valores das Medidas Genéricas . . . . . . . 111

7.10 Regras de $\mathbf{h}_{2}$ Ordenadas pelos Valores das Medidas Relativas. . . . . . . . 111

7.11 Regras de $\mathbf{h}_{1}$ e $\mathbf{h}_{2}$ Ordenadas pelos Valores da Medidas de Interessabilidade 113 


\section{Lista de Algoritmos}

6.1 Cálculo de Informações para Avaliação de Regras Unordered . . . . . . . . 83

6.2 Cálculo de Informações para Avaliação de Regras Ordered . . . . . . . . . . 84

6.3 Cálculo das Informações para um Conjunto de Hipóteses $\mathcal{H}$. . . . . . . . . 87

6.4 Cálculo do Grau de Surpresa de Pequenos Disjuntos . . . . . . . . . . . . . 89 


\section{Lista de Abreviaturas}

Aprendizado de Máquina

$\mathrm{BF}$ Base de Fatos

$\mathrm{DM}$ Data Mining Fertilização In Vitro Generalização Mínima Inteligência Artificial

ICMC Instituto de Ciências Matemáticas e de Computação

ICSI Injeção Intracitoplasmática do Espermatozóide no Oócito

IUI Inseminação Intra Uterina KDD . . . . . . . . . . . . Knowledge Discovery on Databases LABIC . . . . . . . . . . Laboratório de Inteligência Computacional

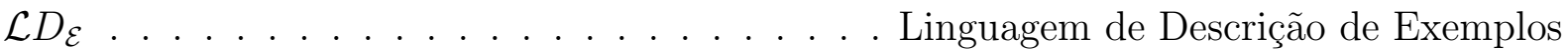

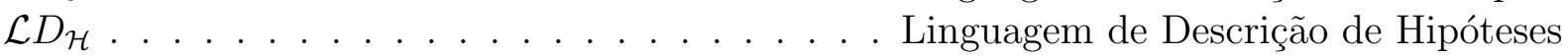

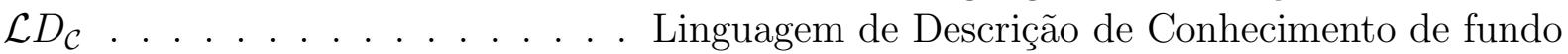

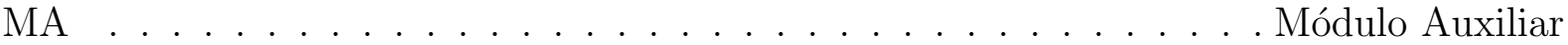
MAR . . . . . . . . . . . . . . Módulo de Análise de Regras MCE . . . . . . . . . . . . Módulo de Combinação e Explicação

$\mathrm{MD}$ Mineração de Dados

MT Mineração de Textos NILC . . . . . . . . Núcleo Interinstitucional de Lingüística Computacional $\mathcal{P B M} \ldots \ldots \ldots \ldots \ldots$ Prati-Baranauskas-Monard PEFD . . . . . . . . . . . Procedures Execution Flow Diagram PLI . . . . . . . . . . . . . . . . . . . Programação Lógica Indutiva USP . . . . . . . . . . . . . . . . . . Universidade de São Paulo 


\section{Capítulo 1}

\section{Introdução}

Com o progresso das tecnologia voltadas para a coleta, armazenamento e disponibilização de dados, surge a necessidade do desenvolvimento de técnicas e ferramentas computacionais capazes de auxiliar a extração de informações úteis (conhecimento) contidas nesses grandes volumes de dados. Assim, o conhecimento pode ser visto como um modelo extraído dos dados ou, ainda, como o produto final de um processo de descoberta orientado pelos dados.

O processo de descoberta de conhecimento de base de dados, i.e., o processo de KDD (Knowledge Discovery on Databases) (Fayyad et al., 1996) tem sido objeto de pesquisa nas áreas de Inteligência Artificial (IA), Estatística e Banco de Dados. O processo de KDD é iterativo, interativo e, resumidamente, envolve três etapas:

1. Seleção, Pré-processamento e Transformação dos dados;

2. Data Mining (Extração do conhecimento);

3. Avaliação e Interpretação do conhecimento extraído.

Data Mining (DM) constitui um campo de pesquisa recente em IA, cujo objetivo é extrair conhecimento de grandes bases de dados. Um dos tópicos tratados em DM para extrair conhecimento é o uso de algoritmos de Aprendizado de Máquina (AM) em grandes volumes de dados.

No modo não supervisionado, o algoritmo de AM é abastecido com um conjunto de exemplos (conjunto de dados) de treinamento que não possuem o rótulo de uma classe. 
Nesse caso, a tarefa do algoritmo é identificar um conjunto finito de categorias, agrupando exemplos através de similaridades.

No modo supervisionado, o algoritmo de AM (indutor) é abastecido com um conjunto de exemplos (conjunto de dados) de treinamento no qual cada exemplo é freqüentemente descrito por um vetor de atributos ou características e uma classe rotulada. Se o rótulo da classe for discreto, tem-se um problema de classificação; se o rótulo for contínuo tem-se um problema de regressão. Tanto um algoritmo de AM supervisionado quanto um não supervisionado podem ser incumbidos de tarefas de descoberta de conhecimento.

Dentro do contexto atual da utilização de algoritmos de AM na etapa de Data Mining, a tarefa de descoberta de conhecimento pode ser vista sob duas formas:

1. Predição, na qual o sistema de AM encontra um modelo com o propósito de predizer, por exemplo, a classe de um novo exemplo.

2. Descrição, na qual o sistema de AM encontra um modelo com o propósito de apresentar uma explicação de forma inteligível ao ser humano. A explicação da representação interna de um algoritmo de AM pode ser apresentada sob uma forma simbólica explícita. Uma dessas formas de representação simbólica explícita do conhecimento é feita através de regras de conhecimento.

O número de regras que constituem uma hipótese (ou classificador) pode ser, especialmente em Data Mining, muito grande. Isso dificulta muito a análise de regras individuais, ou de um grupo de regras, por parte do usuário. No intuito de propor uma solução para essa dificuldade, a análise automática de regras destaca-se como uma das fontes de resultados positivos da aplicação do Aprendizado de Máquina na área de DM.

Várias medidas de avaliação de regras podem ser encontradas na literatura (Freitas, 1998b; Freitas, 1998a; Fayyad et al., 1996; Dean and Famili, 1997). Sob uma perspectiva probabilística, é possível ter uma visão unificada das medidas como precisão, confidência, sensitividade, especificidade, cobertura, suporte, novidade e satisfação. A base padrão para o cálculo de medidas de avaliação de hipóteses são valores extraídos de uma matriz de confusão, enquanto que o cálculo dessas medidas, para uma regra, utiliza freqüências relativas de uma tabela de contingência (Lavrac et al., 1999).

A análise de regras utilizando medidas de avaliação tem a intenção de apontar quais as regras que são melhor sustentadas pelas informações nelas contidas. No entanto, o 
conhecimento descrito por essas regras pode ser óbvio ou irrelevante, mesmo quando elas apresentam um bom desempenho para alguma medida de avaliação.

No intuito de revelar se o conhecimento descoberto pode causar algum surpresa ou despertar algum interesse no usuário, várias medidas de interessabilidade ${ }^{1}$ tem sido estudadas atualmente (Hilderman and Hamilton, 1999). Utilizando essas medidas, é possível analisar o conhecimento simbólico, descrito sob a forma de regras, de acordo com um aspecto subjetivo, ou seja, tomando como base impressões gerais do usuário; ou ainda de acordo com um aspecto objetivo, que utiliza somente os dados que compõem cada regra ou o conjunto delas.

O trabalho aqui apresentado tem o intuito de fornecer subsídios para auxiliar a análise automática de regras, utilizando medidas de avaliação e de interessabilidade de regras. Para realizar essa tarefa, um sistema computacional protótipo, denominado $\mathcal{R}_{\text {ule }} \mathcal{S}_{\text {ystem }}$, foi desenvolvido conjuntamente com Flávia Cristina Bernardini (Bernardini, 2002), utilizando a linguagem de programação lógica Prolog. Esse sistema é composto de dois módulos principais e um módulo auxiliar. Um desses módulos — o Módulo de Análise de Regras (MAR) - é proposto neste trabalho de pesquisa.

Deve ser observado que no Laboratório de Inteligência Computacional - $\mathrm{LABIC}^{2}$ está em desenvolvimento um projeto de grande porte denominado DisCOVER, inicialmente proposto por Baranauskas e Batista (Baranauskas and Batista, 2000). O projeto DisCOVER tem como objetivo fornecer um ambiente integrado para apoiar as etapas do processo de descoberta de conhecimento, oferecendo funcionalidades voltadas para Aprendizado de Máquina (Batista, 1997; Caulkins, 2000; Milaré, 2000; Martins, 2001; Pila, 2000), Mineração de $\operatorname{Dados}^{3}$ (MD) (Batista, 2000; Félix, 1998; Horst, 1999; Lee, 2000; Nagai, 2000; Pugliesi, 2001; Baranauskas, 2001) e Mineração de Textos ${ }^{4}(\mathrm{MT})$ (Imamura, 2001). Dessa forma, as funcionalidades implementadas no $\mathcal{R}_{\text {ule }} \mathcal{S}_{\text {ystem }}$, após serem exaustivamente testadas e validadas, serão implantadas no projeto DisCover.

Os capítulos que compõem este trabalho de pesquisa são sucintamente descritos a seguir: No Capítulo 2 são apresentadas algumas características gerais dos sistemas de

\footnotetext{
${ }^{1} \mathrm{O}$ termo interessabilidade é utilizado neste trabalho como tradução do termo em inglês interestingness.

${ }^{2}$ http://labic.icmc.sc.usp.br

${ }^{3}$ Data Mining

${ }^{4}$ Text Mining
} 
Aprendizado de Máquina, tais como paradigmas, estratégias, modos e tipos de aprendizado. No Capítulo 3 são abordadas algumas medidas propostas na literatura, todas elas baseadas no framework ${ }^{5}$ (Lavrac et al., 1999), que procura unificar as medidas de avaliação de regra sob uma mesma visão, dentro de uma perspectiva probabilística. No Capítulo 4 são apresentadas algumas medidas de interessabilidade bem como os aspectos e princípios de interessabilidade de regras. No Capítulo 5 é apresentado o $\mathcal{R}_{\text {ule }} \mathcal{S}_{\text {ystem }}$ através da descrição do formato dos seus dados de entrada, da sua arquitetura e da metodologia utilizada para documentar o sistema. No Capítulo 6 é apresentado o Módulo de Análise de Regras do $\mathcal{R}_{\text {ule }} \mathcal{S}_{\text {ystem }}$, onde são descritos os procedimentos que implementam as medidas de avaliação apresentadas no Capítulo 3 e as medidas de interessabilidade apresentadas no Capítulo 4. No Capítulo 7 é apresentado um estudo de caso utilizando o MAR. Finalmente, no Capítulo 8, são apresentadas as conclusões inerentes ao trabalho realizado.

\footnotetext{
${ }^{5}$ Optamos pelo termo em inglês já que, quando usado no contexto computacional, o mesmo não possui tradução adequada para a língua portuguesa, sendo, inclusive, largamente utilizado pela comunidade científica.
} 


\section{Capítulo 2}

\section{Aprendizado de Máquina}

\subsection{Considerações Iniciais}

Com o avanço da tecnologia, grandes volumes de dados estão sendo coletados e acumulados numa velocidade espantosa. Data Mining constitui um campo de pesquisas recente em Inteligência Artificial, cujo objetivo é extrair conhecimento de grandes bases de dados. Um dos tópicos tratados em DM para extrair conhecimento é o uso de algoritmos de Aprendizado de Máquina em grandes quantidades de dados.

Segundo Michalski et. al (Michalski et al., 1983), o Aprendizado de Máquina é o campo da IA que se preocupa com o desenvolvimento de teorias computacionais sobre aprendizado e também com a construção de sistemas capazes de adquirir conhecimento de forma automática.

Pesquisas em Aprendizado de Máquina, que inicialmente eram limitadas a estudos teóricos e, posteriormente, a estudos experimentais, recebem atualmente maior reconhecimento em virtude de sua aplicação prática. A aplicação de métodos de AM na busca de soluções para problemas de domínios do mundo real tem sido marcante para a manutenção e maturidade dessas pesquisas.

\subsection{Aprendizado e Aquisição de Conhecimento}

O processo de aprendizado é um fenômeno multifacetado que inclue a aquisição de um novo conhecimento declarativo, o desenvolvimento de habilidades motoras e cognitivas 
através de instruções e de prática, a organização do conhecimento em geral, representações efetivas e a descoberta de novos fatos e teorias através da observação e experimentação. Segundo Michalski et. al (Michalski et al., 1986) o aprendizado pode ser visto basicamente sob duas formas:

1. Refinamento de Habilidades e

2. Aquisição de Conhecimento.

A primeira forma é representada por uma melhora gradual na execução de atividades motoras e cognitivas, através da prática. O aperfeiçoamento do processo de aprendizado consiste em refinar habilidades aprendidas, através da coordenação motora e mental, no intuito de corrigir imperfeições. Por exemplo, quanto mais uma pessoa anda de bicicleta ou joga tênis, melhor fica seu desempenho.

A segunda forma é definida como sendo a capacidade de aprender informações simbólicas aliadas com a habilidade de aplicar aquela informação de modo efetivo. É como aprender física ou matemática. Ao aprender, essa pessoa diz que adquiriu conceitos relevantes, entendeu seus significados e as relações destes com o mundo real. A essência do aprendizado neste caso é a aquisição de novo conhecimento, incluindo descrições e modelos de seus sistemas e procedimentos, incorporando uma variedade de representações procedentes de modelos mentais intuitivos, exemplos e imagens.

Assim, o processo de aprendizado inclui a aquisição de novos conhecimentos, o desenvolvimento das habilidades motoras e cognitivas através de instrução e prática, a organização de novos conhecimentos em geral ou formas efetivas de representar o conhecimento, bem como a descoberta de novos fatos e teorias por meio de observações e experimentações. Considerando que o conhecimento pode ser visto como uma abstração ou um nível de informação acima dos dados, existe a necessidade de áreas de pesquisa dentro da computação que tratem desse assunto, tais como a área de Inteligência Artificial.

Em IA, o processo de aquisição de conhecimento pode ser realizado de forma explícita, ou seja, a aquisição é feita por intermédio de, por exemplo, engenheiros de conhecimento utilizando técnicas convencionais (estudo de casos, entrevistas, etc.). Outra forma de aquisição de conhecimento é a implícita, isto é, de forma automatizada utilizando conjuntos de dados disponíveis no domínio considerado. 
A subárea de IA, chamada de Aprendizado de Máquina, tem como objetivo principal o estudo e a modelagem do processo de aprendizado e suas múltiplas manifestações, realizando aquisição automática de conhecimento.

O Aprendizado de Máquina é fundamental em IA e sua metodologia tem se desenvolvido de acordo com os principais interesses do ramo. Em resposta às dificuldades de codificar volumes de conhecimento sempre crescentes em modernos sistemas de IA, muitos pesquisadores têm voltado sua atenção para AM como meio de vencer o gargalo da aquisição de conhecimento.

Visto de uma maneira prática, o Aprendizado de Máquina pode ser entendido como um conjunto de métodos, técnicas e ferramentas próprias para a aquisição automatizada de conhecimento a partir de conjuntos de dados (Mitchell, 1997).

\subsection{Características Gerais dos Sistemas de Aprendi- zado de Máquina}

Um sistema de aprendizado é um programa de computador que toma decisões baseadas em experiências acumuladas através da solução bem sucedida de problemas anteriores. Os sistemas de Aprendizado de Máquina possuem características particulares e comuns que possibilitam uma certa classificação quanto a linguagem de descrição, modo, paradigma e forma de aprendizado utilizados nesses sistemas, resumidos na Tabela 2.1 por (Félix et al., 1998) e sucintamente descritos a seguir.

\begin{tabular}{|l|l|l|l|}
\hline $\begin{array}{l}\text { Modos de } \\
\text { Aprendizado }\end{array}$ & $\begin{array}{l}\text { Paradigmas } \\
\text { de Aprendizado }\end{array}$ & $\begin{array}{l}\text { Linguagens } \\
\text { de Descrição }\end{array}$ & $\begin{array}{l}\text { Formas de } \\
\text { Aprendizado }\end{array}$ \\
\hline $\begin{array}{l}\text { Supervisionado } D_{\mathcal{E}}-\text { Exemplos ou Objetos } \\
\text { Simbólico }\end{array}$ & Estatístico & $\begin{array}{l}\mathcal{L} D_{\mathcal{H}}-\text { Hipóteses ou } \\
\text { Conceitos Aprendidos } \\
\text { Não } \\
\text { Supervisionado }\end{array}$ & Instance-based \\
Conexionista & $\begin{array}{l}\mathcal{L} D_{\mathcal{C}}-\text { Teoria de Domínio ou } \\
\text { Conhecimento de Fundo }\end{array}$ & $\begin{array}{l}\text { Não Incre- } \\
\text { mental }\end{array}$ \\
& Genético & & \\
\hline
\end{tabular}

Tabela 2.1: Características Gerais de Sistemas de Aprendizado de Máquina. 


\subsubsection{Modos de Aprendizado}

Segundo Russel (Russell and Norvig, 1995), para alguns sistemas de aprendizado é necessário predizer se uma certa ação irá fornecer uma certa saída. Nesse sentido é possível classificar os sistemas de aprendizado de máquina segundo dois modos:

1. aprendizado supervisionado, no qual dado um conjunto de observações ou exemplos rotulados, isto é, a classe de cada exemplo é conhecida, o objetivo é encontrar uma hipóteses capaz classificar novas observações entre as classes já existentes.

2. aprendizado não-supervisionado ou clustering, no qual dado um conjunto de observações ou exemplos não rotulados, o objetivo é tentar estabelecer a existência de clusters ou similaridades nesses dados.

\subsubsection{Paradigmas de Aprendizado}

Atualmente, já foram propostos diversos paradigmas de AM. Esta seção procura apresentar brevemente alguns deles, tais como o paradigma Simbólico, Estatístico, Instancebased, Conexionista e Genético.

\section{Paradigma Simbólico}

Os sistemas de aprendizado simbólico buscam aprender construindo representações simbólicas de um conceito através da análise de exemplos e contra-exemplos desse conceito. As representações simbólicas estão tipicamente na forma de alguma expressão lógica, árvore de decisão, regras de produção ou rede semântica.

Atualmente, entre as representações simbólicas mais estudadas estão as árvores e regras de decisão. É atribuído a Morgan e Messeger (Morgan and Messenger, 1973) o desenvolvimento original do programa para a indução de árvores de decisão. Métodos de indução de árvores de decisão a partir de dados empíricos, conhecido como particionamento recursivo, foram estudados por pesquisadores da área de Inteligência Artificial e Estatística. Os sistemas ID3 (Quinlan, 1986) e C4 (Quinlan, 1987) para indução de árvores de decisão tiveram uma importante contribuição sobre a pesquisa em Inteligência Artificial. O sistema de classificação de árvores de 
regressão CART (Breiman et al., 1984) foi desenvolvido por estatísticos, durante praticamente o mesmo período que o ID3, no final dos anos 70 .

Os trabalhos com indução de regras de decisão surgiram com a simples tradução das árvores de decisão para regras e foi inicialmente tratada em (Quinlan, 1987). Posteriormente, foram criados métodos que induzem regras diretamente a partir dos dados, um desses métodos pode ser encontrado em (Michalski et al., 1983).

\section{Paradigma Estatístico}

Pesquisadores em estatística têm criado diversos métodos de classificação, muitos deles semelhantes aos métodos empregados pela comunidade científica em Aprendizado de Máquina. Por exemplo, o método CART (Breiman et al., 1984), mencionado anteriormente, é um sistema muito conhecido para construir árvores de decisão, desenvolvido por estatísticos. Como regra geral, técnicas estatísticas tendem a focar tarefas em que todos os atributos têm valores contínuos ou ordinais. Muitos deles também são paramétricos, assumindo alguma forma de modelo, e então encontrando valores apropriados para os parâmetros do modelo a partir dos dados. Por exemplo, um classificador linear assume que as classes podem ser expressas como combinação linear dos valores dos atributos, e então procura uma combinação linear particular que fornecer a melhor aproximação sobre o conjunto de dados. Os classificadores estatísticos freqüentemente assumem que valores de atributos estão normalmente distribuídos, e então usam os dados fornecidos para determinar média, variância e co-variância da distribuição.

Alguns autores têm considerado redes neurais como métodos estatísticos paramétricos, uma vez que treinar uma rede neural geralmente significa encontrar valores apropriados para pesos e bias pré-determinados.

\section{Paradigma Instance-based}

Uma forma de classificar um caso é lembrar de um caso similar cuja classe é conhecida e assumir que o novo caso terá a mesma classe. Essa filosofia exemplifica os sistemas instance-based, que classificam casos nunca vistos através de casos similares conhecidos (Quinlan, 1993).

Saber quais casos de treinamento devem ser memorizados é importante para evitar 
dificuldades e lentidão de manuseio por parte do classificador. O ideal é reter apenas os casos com os quais seja possível resumir toda a informação. Em (Aha et al., 1991) estão descritas algumas estratégias para decidir quando um novo caso deve ser memorizado. A medida de similaridade para os casos nos quais todos os atributos são contínuos pode ser calculada através de alguma distância entre esses atributos. $\mathrm{Na}$ presença de atributos ordinais esta medida se torna complicada, bem como na presença de atributos irrelevantes, os quais podem fazer com que dois casos similares sejam interpretados como muito diferentes. Métodos sensíveis ao contexto que alterem a escala dos atributos podem melhorar estas medidas (Stanfill and Waltz, 1986)

\section{Paradigma Conexionista}

Redes neurais são construções matemáticas relativamente simples que foram inspiradas no modelo biológico do sistema nervoso. A representação de uma rede neural envolve unidades altamente interconectadas e, por esse motivo, o nome conexionismo é utilizado para descrever a área de estudo.

As pesquisas em redes neurais foram iniciadas com o trabalho pioneiro de McCulloch e Pitts em (McCulloch and Pitts, 1943). McCulloch era um psiquiatra e pesquisou por 20 anos uma forma de representar um evento no sistema nervoso. Pitts era um jovem pesquisador e começou a trabalhar com McCulloch em 1942. Praticamente 15 anos após a publicação de McCulloch e Pitts, Rosenblatt em (Rosemblatt, 1985) apresentou o perceptron, cuja grande contribuição foi a prova do teorema de convergência. Mas no livro Perceptrons (Minsky, 1969), Minsky e Papert demonstraram a existência de limites fundamentais nos perceptrons de uma camada.

A pesquisa na área ficou praticamente estática até que Hopfield em (Hopfield, 1982) utilizou a idéia de uma função de energia para formular uma nova forma de compreender os cálculos realizados em redes recorrentes com conexões sinápticas simétricas. Na década de 80, o artigo de Hopfield em 1982 e o livro de Rumelhart e McLelland (Rumelhart and McClelland, 1986) foram as publicações que mais influenciaram para o resurgimento do interesse sobre redes neurais na década de 80 . Redes neurais tiveram um longo caminho desde McCulloch e Pitts, e continuam a crescer em teoria, projetos e aplicações (Braga et al., 2000). 
A metáfora biológica com as conexões neurais do sistema nervoso tem interessado muitos pesquisadores e tem fornecido muitas discussões sobre os méritos e as limitações dessa abordagem de aprendizado. Em particular, as analogias com a biologia têm levado muitos pesquisadores a acreditar que as redes neurais possuem um grande potencial na resolução de problemas que requerem intenso processamento sensorial humano, tal como visão e reconhecimento de voz e imagens.

\section{Paradigma Genético}

Este formalismo de classificação é derivado do modelo evolucionário de aprendizado (Holland, 1986). Um classificador genético consiste de uma população de elementos de classificação que competem para fazer a predição. Elementos que possuem uma performance fraca são descartados, enquanto os elementos mais fortes proliferam, produzindo variações de si mesmos. Este paradigma possue uma analogia direta com a teoria de Darwin, na qual sobrevivem os mais bem adaptados ao ambiente.

Segundo Goldberg (Goldberg, 1989)

Um algoritmo genético é um procedimento iterativo que mantém uma população de indivíduos, cada um dos quais é um candidato à solução de algum problema específico. A cada iteração (denominada geração), os indivíduos da população atual são avaliados quanto a sua aptidão para a solução do problema. Com base nessas avaliações aplicam-se alguns operadores genéticos aos indivíduos, formando-se uma nova população, que substituirá a população atual. Isto é feito de modo que, quanto maior a aptidão de um indivíduo da população atual, maior a sua influência na formação dos indivíduos da nova geração. Assim, com o passar do tempo, a seleção natural tende a fazer com que a população seja formada por indivíduos cada vez melhores (soluções cada vez mais próximas da solução ótima para o problema). Como critério de parada do algoritmo genético, geralmente define-se um limite no número de gerações. Dentre os indivíduos da última geração, aqueles mais aptos representam a melhor solução encontrada pelo algoritmo. Pode-se também especificar que o algoritmo encerrará quando for gerado algum indivíduo que satisfaça alguma condição mínima de aptidão. 
Alguns operadores genéticos básicos que aplicados a população geram novos indivíduos são: Reprodução, Cruzamento, Mutação e Inversão. Esses operadores atuam no controle da quantidade de cópias produzidas de um indivíduo, na troca de material genético, na preservação de uma espécie e na manutenção de uma certa diversidade na nova população (Freitas and Kirner, 1992).

\subsubsection{Linguagens de Descrição}

$\mathrm{Na}$ aquisição de conhecimento, um dos principais interesses está na derivação de descrições simbólicas que são orientadas aos humanos, isto é, que sejam fáceis de entender e fáceis de usar para criar modelos mentais da informação que elas trazem consigo.

Nesse caso, o resultado de uma indução por computador deve ser sob a forma de descrições simbólicas de entidades dadas, semanticamente e estruturalmente similar ao que um especialista humano pode produzir observando as mesmas entidades. Componentes dessas descrições podem ser compreensíveis como simples "pedaços" da informação, diretamente interpretável em linguagem natural, e podem relatar quantitativamente e qualitativamente os componentes dessa informação em um estilo integrado (Michalski et al., 1983).

Qualquer que seja o tipo de aprendizado, é necessário uma linguagem para descrever objetos (ou possíveis eventos), uma linguagem para descrever conceitos, ou hipóteses, e uma para descrever conhecimento de fundo.

1. Linguagem de Descrição de Exemplos (Objetos) $-\mathcal{L} D_{\mathcal{E}}$.

- Descrições Estruturais

Uma descrição estrutural retrata um objeto como sendo uma estrutura complexa consistindo de vários componentes além das relações entre eles. Por exemplo, a descrição estrutural de um arco pode ser:

Um arco consiste de três componentes - dois postes e um lintel, tal que cada um deles é um bloco; os dois postes suportam o lintel; os postes estão na vertical, são paralelos, não se tocam e o lintel está na horizontal..

- Descrições de Atributos 
Em uma descrição de atributos, um objeto é descrito em termos de suas características globais como um vetor de valores de atributos. Uma descrição de atributos de um arco pode ser:

Seu tamanho é $8 \mathrm{~cm}$, sua altura é $5 \mathrm{~cm}$ e sua cor é amarelo.

A princípio, um atributo pode ser de qualquer tipo, numérico ou não numérico.

2. Linguagem de Descrição de conceitos (Hipóteses) - $\mathcal{L} D_{\mathcal{H}}$. Os formalismos freqüentemente usados em AM para descrever conceitos são:

- regras se-então (if-then),

- árvores de decisão,

- lógica de predicados (filha $(X, Y)<--\operatorname{mulher}(X)$, pais $(X, Y))$,

- redes semânticas.

3. Linguagem de Descrição da teoria do domínio (Conhecimento de Fundo) $-\mathcal{L} D_{\mathcal{C}}$. Utilizada para descrever algum conhecimento prévio e relevante do domínio do problema.

\subsubsection{Aprendizado Incremental e Não-Incremental}

Os algoritmos de aprendizado podem ser classificados de duas formas segundo o modo em que os exemplos são apresentados

1. não incremental que necessita que todos os exemplos, simultaneamente, estejam disponíveis para que seja induzido um conceito. É vantajoso usar esses algoritmos para problemas de aprendizado nos quais todos os exemplos estão disponíveis e, provavelmente, não irão ocorrer mudanças.

2. incremental que revê a definição do conceito corrente, se necessário, em resposta a cada novo exemplo de treinamento observado. Os exemplos observados são considerados um a um pelo sistema, isto é, o sistema considera o primeiro exemplo e, de acordo com este exemplo constrói uma determinada hipótese; a seguir considera um segundo exemplo, que pode ou não modificar a primeira hipótese, baseandose em como esta classifica o segundo exemplo. Dessa forma, o sistema continua modificando o conceito à medida que mais exemplos são apresentados. 
Uma das vantagens de usar um algoritmo incremental é que o conhecimento pode ser rapidamente atualizado a cada nova observação. Pode ser mais eficiente revisar uma hipótese existente do que gerar uma nova hipótese cada vez que um novo exemplo é observado (Utgoff, 1989).

\subsection{Sistemas de Aprendizado de Máquina Indutivo}

A inferência indutiva é um dos principais meios de descobrir novo conhecimento e prever eventos futuros. Neste tipo de aprendizado são criadas generalizações, a partir de um conjunto de exemplos de um dado domínio, com a intenção de descrever um conceito aprendido ou predizer uma ação futura.

Os sistemas de AM capazes de criar generalizações ou descrever conceitos a partir de um conjunto de dados externos, são conhecidos como sistemas de Aprendizado de Máquina indutivo ou apenas indutores. Neste trabalho são utilizados indutores nos quais os exemplos estão no formato atributo-valor e induzem classificadores simbólicos, como descrito a seguir.

\subsubsection{Exemplos no Formato Atributo-Valor}

O indutor é abastecido com um conjunto de exemplos de treinamento $S$ rotulados, com $N$ exemplos $T_{i}, i=1, \ldots, N$, escolhidos de um domínio $X$ com uma distribuição $D$ fixa, desconhecida e arbitrária, da forma $\left\{\left(\mathbf{x}_{1}, y_{1}\right), \ldots,\left(\mathbf{x}_{N}, y_{N}\right)\right\}$ para alguma função desconhecida $y=f(\mathbf{x})$, como mostra a Tabela 2.2. Os $\mathbf{x}_{i}$ são tipicamente vetores da forma $<x_{i 1}, x_{i 2}, \ldots, x_{i M}>$, com valores discretos ou contínuos. Assim, $x_{i j}$ refere-se ao valor do atributo (ou feature) $j$, denominado $\mathbf{X}_{j}$, do exemplo $T_{i}$, como mostra a Tabela 2.2. Os valores $y_{i}$ referem-se ao valor do atributo $Y$, freqüentemente denominado classe.

\begin{tabular}{c|cccc|c|}
\cline { 2 - 6 }$T_{1}$ & $X_{1}$ & $X_{2}$ & $\ldots$ & $X_{M}$ & $Y$ \\
\cline { 2 - 6 }$T_{2}$ & $x_{11}$ & $x_{12}$ & $\ldots$ & $x_{1 M}$ & $y_{1}$ \\
$\vdots$ & $x_{21}$ & $x_{22}$ & $\ldots$ & $x_{2 M}$ & $y_{2}$ \\
$T_{N}$ & $\vdots$ & $\vdots$ & $\ddots$ & $\vdots$ & $\vdots$ \\
\cline { 2 - 5 } & $x_{N 1}$ & $x_{N 2}$ & $\ldots$ & $x_{N M}$ & $y_{N}$ \\
\hline
\end{tabular}

Tabela 2.2: Conjunto de Exemplos Rotulados no Formato Atributo-Valor 
Os valores $y_{i}$ são tipicamente pertencentes a um conjunto discreto de classes $C_{v}, v=$ $1, \ldots, N C l$, da forma $\left\{C_{1}, \ldots, C_{N C l}\right\}$, quando se trata de classificação ou ao conjunto de números reais (valores contínuos) no caso de regressão.

Dado um conjunto $S$ de exemplos de treinamento a um indutor, a sua tarefa é induzir um classificador $\mathbf{h}$. O classificador consiste da hipótese feita sobre a verdadeira (mas desconhecida) função $f$. Dados novos exemplos $\mathbf{x}$, o classificador, ou hipótese, h prediz o valor correspondente $y$.

\subsubsection{Classificadores Simbólicos}

Existem duas formas básicas de ver um classificador $\mathbf{h}$. A primeira é uma visão genérica na qual a hipótese induzida $\mathbf{h}$ representa o conhecimento adquirido visto como uma "caixa preta" - Figura 2.1. Nesse caso não está sendo considerado o paradigma de aprendizado nem a linguagem de descrição da hipótese $\mathbf{h}$.

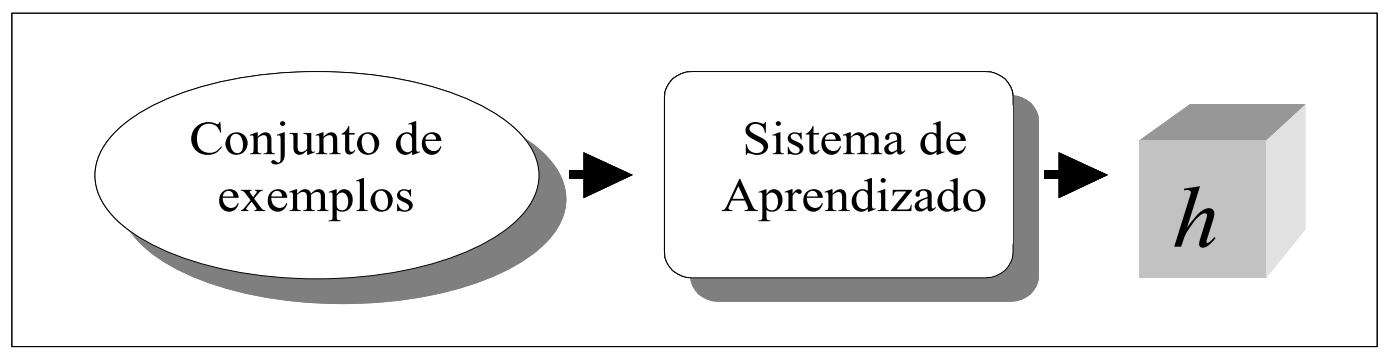

Figura 2.1: h vista como caixa preta

A segunda é uma visão mais apurada, onde a "caixa preta" pode ser aberta - Figura 2.2. Nesse caso, é levado em conta o paradigma de aprendizado e a linguagem de descrição na qual o conhecimento adquirido é expresso.

Quando o conceito descrito por $\mathbf{h}$ é facilmente interpretável por seres humanos, geralmente, $\mathbf{h}$ pode ser transformado em conjuntos de regras if-then, ou seja, regras do tipo Corpo $\rightarrow$ Cabeça ou Body $\rightarrow$ Head. Neste caso, $\mathbf{h}$ consiste de um conjunto de regras com $N R$ regras $R_{u}, u=1, \ldots, N R$, ou seja, $\mathbf{h}=\left\{R_{1}, \ldots, R_{N R}\right\}$, ao qual denominamos classificador simbólico. Entretanto, o número de regras que constituem uma hipótese h pode ser muito grande. Isso dificulta muito a análise de regras individuais ou de um grupo de regras por parte do usuário. 


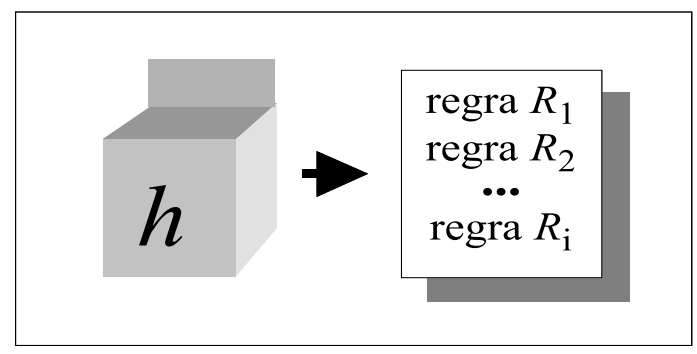

Figura 2.2: $\mathbf{h}$ vista como conjunto de regras

Uma solução possível para essa dificuldade é avaliar um conjunto de regras $\mathbf{h}$ dado um conjunto de exemplos $S$. Neste caso, além de medir a precisão de $\mathbf{h}$ como um todo, isto é, como um classificador tipo caixa preta, é possível avaliar separadamente cada uma das regras que constituem $\mathbf{h}$.

A análise automática de regras destaca-se como uma das fontes de resultados positivos da aplicação do Aprendizado de Máquina na área de Data Mining. Nesse contexto, regras podem ser avaliadas com o objetivo de saber quais são aquelas melhor sustentadas pelos dados - aspecto qualitativo - ou ainda podem ser avaliadas com o intuito de selecionar aquelas que possam trazer algum conhecimento surpreendente ou inesperado para o usuário, ou seja, o objetivo é selecionar as regras mais interessantes — aspecto quantitativo.

\subsection{Considerações Finais}

Nesse capítulo foram apresentadas algumas das características gerais de sistemas de aprendizado de máquina, tais como modos e paradigmas de aprendizado, tipos de aprendizado e as linguagens de descrição necessárias.

Foi também descrita a linguagem de descrição $\mathcal{L} D_{\mathcal{E}}$ de exemplos bem como a linguagem de descrição $\mathcal{L} D_{\mathcal{H}}$ de hipóteses, ou conceitos, aprendidos pelos indutores utilizados neste trabalho.

Aprendizado de Máquina é uma área de pesquisa que tem alcançado muitos resultados positivos ao ser aplicado em Data Mining. No entanto, ainda existem inúmeras questões importantes a serem investigadas como, por exemplo, questões referentes à análise qualitativa de regras de conhecimento. Para efetuar esse tipo de análise podem ser utilizadas 
medidas de avaliação de regras, tais como as apresentadas no próximo capítulo. 


\section{Capítulo 3}

\section{Medidas de Avaliação de Regras}

\subsection{Considerações Iniciais}

Geralmente, quando o indutor é orientado para tarefas descritivas de descoberta de conhecimento, o volume de regras induzidas pode ser muito grande, o que dificulta a análise do poder descritivo dessas regras por parte do usuário.

Numerosas medidas de avaliação apresentadas na literatura (Piatetsky-Shapiro, 1991; Dean and Famili, 1997) utilizam técnicas estatísticas para avaliar a capacidade de representação do conhecimento adquirido sob a forma de regras. Essas medidas podem ser utilizadas para efetuar uma análise de como essas regras são sustentadas pelos exemplos, ou seja, essas medidas podem ser utilizadas para efetuar uma análise qualitativa das regras (Horst, 1999; Horst and Monard, 2000).

Nesse capítulo são apresentadas algumas medidas propostas na literatura, baseadas em um framework ${ }^{1}$ que procura unificar, sob uma mesma visão, as medidas de avaliação de regras (Lavrac et al., 1999). Nesse framework, as definições são dadas em termos de freqüências relativas derivadas da tabela de contingência da regra.

\footnotetext{
${ }^{1}$ Optamos pelo termo em inglês, largamente utilizado pela comunidade acadêmica, pois quando usado no contexto computacional, o mesmo não possui uma tradução adequada para a língua portuguesa.
} 


\subsection{Notação e Terminologia}

Nessa seção é apresentada a notação e a terminologia que será utilizada ao longo deste trabalho, considerando um formato genérico para as regras induzidas.

\subsubsection{Regras}

Neste trabalho são considerados sistemas de AM que induzem regras de conhecimento na forma

$$
\text { Corpo } \rightarrow \text { Cabeça }
$$

ou, em inglês:

$$
\text { Body } \rightarrow \text { Head }
$$

Será aqui adotada a forma Body $\rightarrow$ Head pois essa permite ser resumidamente denotada como $B \rightarrow H$, o que não acontece com a forma Corpo $\rightarrow$ Cabeça.

Medidas de avaliação de regras pretendem dar uma indicação da força (hipotética) de associação entre Head e Body expressa por uma regra. É também assumido que, dado um conjunto de exemplos rotulados, existe um procedimento pelo qual é possível determinar, para cada possível Head e Body, se Head é ou não verdade e se Body é ou não verdade para um determinado exemplo.

Essas regras preditivas podem ser induzidas por sistemas de aprendizado proposicional ou de primeira ordem. No caso de regras proposicionais, Body é tipicamente uma conjunção de pares atributo-valor e Head é uma designação da classe. No caso do aprendizado de primeira ordem, freqüentemente denominada de Programação Lógica Indutiva (PLI) (Lavrac and Dzeroski, 1994), as regras são cláusulas Prolog nas quais Head é um literal positivo e Body é uma conjunção de literais positivos e/ou negativos. Regras de primeira ordem contêm variáveis que são compartilhadas entre os literais e entre Head e Body, diferenciando-se assim das regras proposicionais.

É dito que um exemplo é coberto por uma regra Body $\rightarrow$ Head se Body é verdade para esse exemplo. No caso proposicional, um exemplo é coberto quando ele satisfaz as condições de uma regra (todas as condições de uma regra são avaliadas verdadeiras dada a descrição do exemplo). No caso de primeira ordem, o(s) átomo(s) que descrevem os 
exemplos são combinados com a cabeça da regra, determinando assim a substituição $\theta$ pela qual as variáveis na cabeça da regra são substituidas pelos termos (constantes) na descrição do exemplo. A regra cobre o exemplo se e somente se Body $\theta$ é avaliado como verdadeiro.

Este trabalho concentra-se em regras induzidas por sistemas de aprendizado proposicional ou de primeira ordem.

\subsubsection{Matriz de Confusão}

Como mencionado anteriormente, o classificador induzido por um algoritmo de AM pode ser considerado como uma caixa preta. Neste trabalho, o termo matriz de confusão refere-se ao classificador, enquanto que tabela de contingência, definida na Seção 3.2.3, refere-se a uma única regra. Ambos conceitos são semelhantes mas, no primeiro caso, é considerada a hipótese induzida (classificador), enquanto, no segundo, somente cada regra que faz parte da hipótese induzida é considerada.

Uma matriz de confusão mostra o número de classificações corretas em oposição às classificações preditas para cada classe. Considerando problemas de classificação binária, ou seja, problemas de classificação com apenas duas classes (geralmente rotuladas como "+ " e " - "), as escolhas são estruturadas para predizer a ocorrência ou não-ocorrência de um simples evento ou hipótese (Baranauskas and Monard, 2000). Quando apenas duas classes são consideradas, os dois erros possíveis são denominados de falsos positivos e falsos negativos.

A Tabela 3.1 ilustra a matriz de confusão para problemas de classificação com duas classes onde $T p$ é o número de exemplos corretamente classificados como exemplos positivos, $F p$ é o número de exemplos erroneamente classificados como positivos, Tn é o número de exemplos corretamente classificados como exemplos negativos, $F n$ é o número de exemplos erroneamente classificados como negativos e $n=(T p+F n+F p+T n)$ é o total de exemplos.

Ainda considerando a Tabela 3.1, quatro situações podem ocorrer:

1. O exemplo pertence à classe $C_{+}$e é predito pelo classificador como pertencente à classe $C_{+}$. Neste caso, o exemplo é um verdadeiro positivo.

2. O exemplo pertence à classe $C_{-}$e é predito pelo classificador como pertencente à 


\begin{tabular}{|c|cc|cc|}
\hline Classe & preditos como $C_{+}$ & preditos como $C_{-}$ & Precisão da Classe & Precisão Total \\
\hline \multirow{4}{*}{$C_{+}$} & Verdadeiros positivos & Falsos negativos & $\frac{T p}{T p+F n}$ & \\
& $T p$ & $F n$ & $\frac{T p+T n}{n}$ \\
$C_{-}$ & Falsos positivos & Verdadeiros negativos & $\frac{T n}{F p+T n}$ & \\
\hline
\end{tabular}

Tabela 3.1: Matriz de Confusão para Problemas de Classificação Binária

classe $C_{-}$. Neste caso, o exemplo é um verdadeiro negativo.

3. O exemplo pertence à classe $C_{-}$e é predito pelo classificador como pertencente à classe $C_{+}$. Neste caso, o exemplo é um falso positivo.

4. O exemplo pertence à classe $C_{+}$e é predito pelo classificador como pertencente à classe $C_{-}$. Neste caso, o exemplo é um falso negativo.

\subsubsection{Tabela de Contingência}

As medidas apresentadas neste trabalho utilizam como base uma tabela de contingência para uma regra arbitrária $R$. Considerando o formato de regras apresentado na seção 3.2.1, $R$ passa a ser aqui resumidamente denotada por $B \rightarrow H$. Na tabela de contingência mostrada na Tabela $3.2, B$ denota o conjunto de exemplos para os quais o corpo da regra é verdade e $\bar{B}$ denota seu complemento, ou seja, o conjunto de exemplos para os quais o corpo da regra é falso; $H$ e $\bar{H}$ referem-se similarmente à cabeça da regra. $H B$ denota então $H \cap B, \bar{H} B$ denota $\bar{H} \cap B$, e assim por diante.

Seja $x$ a cardinalidade do conjunto $X$, ou seja, $x=|X|$. Na Tabela 3.2, $h$ denota a cardinalidade do conjunto $H$, ou seja, $h=|H|$. Em outras palavras, $h$ denota o número de exemplos para os quais a cabeça da regra é verdade. Similarmente $b=|B|$ denota o número de exemplos para os quais o corpo da regra é verdade. Assim, $\bar{h} b=|\bar{H} B|$ denota o número de exemplos para os quais a cabeça é falsa e o corpo é verdade, ou seja, é o número de exemplos erroneamente cobertos pela regra. A cardinalidade $n$ denota o número total de exemplos utilizados para determinar as demais cardinalidades expressas na tabela de contingência.

Associada a cardinalidade $x$, a freqüência relativa $f_{x}$ é utilizada como uma estimativa da probabilidade $P(X)$, ou seja, $P(X)=f_{x}=\frac{x}{n}$. Por exemplo, considerando a 


\begin{tabular}{cccc}
\multicolumn{4}{c}{ Tabela de Contingência } \\
\hline & $H$ & $\bar{H}$ & \\
\hline$B$ & $h b$ & $\bar{h} b$ & $b$ \\
$\bar{B}$ & $h \bar{b}$ & $\overline{h b}$ & $\bar{b}$ \\
& $h$ & $\bar{h}$ & $n$ \\
\hline
\end{tabular}

$h b=$ número de exemplos para os quais $H$ é verdade e $B$ é verdade

$\bar{h} b=$ número de exemplos para os quais $H$ é falso e $B$ é verdade

$h \bar{b}=$ número de exemplos para os quais $H$ é verdade e $B$ é falso

$\overline{h b}=$ número de exemplos para os quais $H$ é falso e $B$ é falso

$b=$ número de exemplos para os quais $B$ é verdade

$\bar{b}=$ número de exemplos para os quais $B$ é falso

$h=$ número de exemplos para os quais $H$ é verdade

$\bar{h}=$ número de exemplos para os quais $H$ é falso

$n=$ número total de exemplos

Tabela 3.2: Tabela de Contingência para uma Regra

cardinalidade $h b$, a probabilidade $P(H B)$ pode ser determinada da seguinte forma:

$$
P(H B)=f_{b h}=\frac{h b}{n}
$$

De forma semelhante podem ser determinados os valores das probabilidades $P(\bar{H} B), P(\overline{H B})$ e $P(H \bar{B})$. Conhecidas essas probabilidades, os valores de $P(B), P(\bar{B}), P(H)$ e $P(\bar{H})$ podem ser determinados. Por exemplo:

$$
P(B)=P(\bar{H} B)+P(H B) \text { ou } P(\bar{B})=P(H \bar{B})+P(\overline{H B})
$$

Todas as medidas de avaliação de regras consideradas neste trabalho estão definidas em termos de estimativas de probabilidade (freqüências relativas) procedentes da tabela de contingência. Note que a tabela de contingência é uma generalização da matriz de confusão, que é a base padrão para computar-se medidas de avaliação de hipóteses em problemas de classificação binária. 


\subsection{Medidas de Avaliação de Regras}

As definições das medidas de avaliação de regras são dadas em termos de freqüências relativas derivadas da tabela de contingência. Algumas dessas medidas são baseadas na medida proposta em (Piatetsky-Shapiro, 1991), que detém a capacidade de trazer consigo alguma novidade.

\subsubsection{Um Exemplo para Avaliação}

Com a finalidade de ilustrar as medidas de avaliação apresentadas a seguir, será considerado o conjunto de exemplos ilustrado na Tabela 3.3, adaptado de (Quinlan, 1993) por (Baranauskas and Monard, 2000). Esse conjunto de dados contém informações sobre medições diárias de condições meteorológicas e uma classificação se o dia é apropriado a uma visita à fazenda ("go") ou não ("dont_go"). Cada exemplo é composto pelas seguintes atributos:

- outlook: assume os valores sunny, overcast ou rain;

- temperature: um valor numérico que indica a temperatura em graus Celsius;

- humidity: um valor numérico que indica a umidade relativa do ar;

- windy: assume os valores yes ou no.

Utilizando o conjunto de dados na Tabela 3.3 como conjunto de treinamento, foi executado o algoritmo C4.5rules (Quinlan, 1993) para induzir regras, as quais são mostradas na Tabela 3.4 na página oposta. Três dessas regras foram selecionadas para ilustrar as medidas apresentadas.

Cada uma dessas regras foi avaliada utilizando como conjunto de teste os exemplos da Tabela 3.5 na página 26. A Tabela 3.6 na página 26 , mostra as três regras selecionadas bem como a tabela de contingência para cada uma dessas regras.

\subsubsection{Medidas Genéricas de Regras}

\section{Precisão, Erro e Confiança Negativa}

Definição 3.3.1. Precisão: $A c c(B \rightarrow H)=P(H \mid B)$ 


\begin{tabular}{ccccc}
\hline \hline Outlook & Temperature & Humidity & Windy & Voyage? \\
\hline rain & 27 & 95 & no & go \\
rain & 20 & 70 & yes & dont_go \\
rain & 23 & 80 & yes & dont_go \\
rain & 25 & 81 & no & go \\
rain & 21 & 80 & no & go \\
sunny & 25 & 72 & yes & go \\
sunny & 21 & 79 & yes & dont_go \\
sunny & 26 & 70 & no & go \\
sunny & 27 & 92 & no & dont_go \\
sunny & 30 & 88 & no & dont_go \\
overcast & 23 & 90 & yes & go \\
overcast & 29 & 78 & no & go \\
overcast & 19 & 65 & yes & dont_go \\
overcast & 26 & 75 & no & go \\
overcast & 20 & 87 & yes & dont_go \\
\hline \hline
\end{tabular}

Tabela 3.3: Conjunto de Exemplos de Treinamento voyage

\begin{tabular}{l}
\hline IF outlook $=$ overcast \\
THEN CLASS $=$ go \\
IF outlook $=$ sunny \\
AND humidity $\leq 78$ \\
THEN CLASS $=$ go \\
IF outlook $=$ sunny \\
AND humidity $>78$ \\
THEN CLASS $=$ dont_go \\
IF outlook $=$ rain \\
AND windy $=$ yes \\
THEN CLASS $=$ dont_go \\
IF outlook $=$ rain \\
AND windy $=$ no \\
THEN CLASS $=$ go \\
CLASS = go
\end{tabular}

Tabela 3.4: Regras Induzidas a partir do Conjunto de Exemplos de Treinamento voyage 


\begin{tabular}{ccccc}
\hline \hline Outlook & Temperature & Humidity & Windy & Voyage? \\
\hline sunny & 25 & 72 & yes & go \\
sunny & 28 & 91 & yes & dont_go \\
sunny & 22 & 70 & no & go \\
sunny & 23 & 95 & no & dont_go \\
sunny & 30 & 85 & no & dont_go \\
overcast & 23 & 90 & yes & go \\
overcast & 29 & 78 & no & go \\
overcast & 19 & 65 & yes & dont_go \\
overcast & 26 & 75 & no & go \\
overcast & 20 & 87 & yes & go \\
rain & 22 & 95 & yes & go \\
rain & 19 & 70 & yes & dont_go \\
rain & 23 & 80 & yes & dont_go \\
rain & 25 & 81 & no & go \\
rain & 21 & 80 & no & go \\
\hline \hline
\end{tabular}

Tabela 3.5: Conjunto de Exemplos de Teste voyage

\begin{tabular}{|llcccc|}
\hline \hline & & & & \\
R1 & IF outlook = overcast & 4 & 1 & 5 \\
& THEN CLASS = go & 5 & 5 & 10 \\
& & 9 & 6 & 15 \\
R2 & IF outlook = sunny & & & \\
& AND humidity $>78$ & 3 & 0 & 3 \\
& THEN CLASS $=$ dont_go & 6 & 9 & 15 \\
& & & & \\
R3 & IF outlook $=$ rain & 2 & 0 & 2 \\
& AND windy $=$ no & 7 & 6 & 13 \\
& THEN CLASS = go & 9 & 6 & 15 \\
& & & & \\
\hline \hline
\end{tabular}

Tabela 3.6: Regras Selecionadas e suas Tabelas de Contingência 
Definição 3.3.2. Erro: $\operatorname{Err}(B \rightarrow H)=P(\bar{H} \mid B)$

A precisão de uma regra $R=B \rightarrow H$ é uma medida do quanto uma regra é específica para o problema. Dentro do framework proposto em (Lavrac et al., 1999), é definida como a probabilidade condicional de $H$ ser verdade dado que $B$ é verdade. Assim:

$$
A c c(R)=P(H \mid B)=\frac{P(H B)}{P(B)}=\frac{f_{h b}}{f_{b}}=\frac{h b}{b}
$$

No caso de problemas de classificação binária, a precisão de uma regra mede a fração de exemplos preditos positivos que são verdadeiros positivos. Quanto maior o valor dessa medida, mais precisamente a regra cobre corretamente os exemplos da sua classe.

O erro de uma regra é dado por:

$$
\operatorname{Err}(R)=1-A c c(R)=1-P(H \mid B)=\frac{1-P(H B)}{P(B)}=\frac{P(\bar{H} B)}{P(B)}=P(\bar{H} \mid B)
$$

Quanto maior o erro, menos precisamente a regra cobre corretamente os exemplos da sua classe.

Considerando as regras $R_{1}, R_{2}$ e $R_{3}$ da Tabela 3.6, a precisão e o erro de ambas as regras resulta respectivamente em:

$$
\begin{array}{ll}
\operatorname{Acc}\left(R_{1}\right)=\frac{4}{5}=0.800 & \operatorname{Err}\left(R_{1}\right)=1-\operatorname{Acc}\left(R_{1}\right)=0.200 \\
\operatorname{Acc}\left(R_{2}\right)=\frac{3}{3}=1.000 & \operatorname{Err}\left(R_{2}\right)=1-\operatorname{Acc}\left(R_{2}\right)=0.000 \\
\operatorname{Acc}\left(R_{3}\right)=\frac{2}{2}=1.000 & \operatorname{Err}\left(R_{3}\right)=1-\operatorname{Acc}\left(R_{3}\right)=0.000
\end{array}
$$

A definição da precisão de uma regra destina-se a avaliar regras individuais e por essa razão tende a favorecer a precisão de exemplos positivos. Como tal, é diferente da precisão do conjunto de regras que constituem a hipótese (classificador), definida em função da matriz de confusão — Seção 3.2.2.

No exemplo considerado, a hipótese (classificador) induzida pelo C4.5rules consiste do conjunto de cinco regras ilustradas na Tabela 3.4, mais a regra default CLASS $=$ go, que é utilizada para classificar exemplos que não são cobertos pelas cinco regras anteriores. A Tabela 3.7 mostra a matriz de confusão desse classificador para o conjunto de exemplos de 
teste na Tabela 3.5, considerando a classe "go" como classe positiva e classe "dont_go" com negativa.

\begin{tabular}{|c|cc|}
\hline Classe & preditos como "go" & preditos como "dont_go" \\
\hline & Verdadeiros positivos & Falsos negativos \\
"go" & Tp=8 & Fn $=1$ \\
& Falsos positivos & Verdadeiros negativos \\
"dont_go" & $F p=1$ & Tn $=5$ \\
\hline
\end{tabular}

Tabela 3.7: Matriz de Confusão

A precisão do conjunto de regras (classificador) é usada como medida padrão para avaliar hipóteses. Ela é dada pela fórmula:

$$
A C(\mathbf{h})=\frac{T p+T n}{n}
$$

Para a matriz de confusão da Tabela 3.7, o valor da precisão da hipótese (ou precisão do classificador) é:

$$
A C(\mathbf{h})=\frac{8+5}{15}=0.867
$$

Dada essa estrutura geral de descoberta de conhecimento, também pode ser visto que a precisão de uma regra é de fato o mesmo que a confidência no aprendizado de regras de associação ${ }^{2}$. A precisão de uma regra pode ser usada também para medir a confiança de uma regra na predição de casos positivos, pois ela mede a corretude dos resultados retornados.

Definição 3.3.3. Confiança negativa: $\operatorname{NegRel}(B \rightarrow H)=P(\bar{H} \mid \bar{B})$

A confiança de predições negativas ou confiança negativa de uma regra é o correspondente à precisão, mas para os exemplos que não são cobertos pela regra. Ela é dada pela fórmula:

$$
\operatorname{NegRel}(R)=P(\bar{H} \mid \bar{B})=\frac{P(\overline{H B})}{P(\bar{B})}=\frac{f_{\overline{h b}}}{f_{\bar{b}}}=\frac{\overline{h b}}{\bar{b}}
$$

\footnotetext{
${ }^{2}$ Regras de associação admitem que Head seja uma conjunção de atributos.
} 
Para problemas de classificação binária, a confiança negativa da hipótese é computada como:

$$
\operatorname{NegRel}(\mathbf{h})=\frac{T n}{T n+F n}
$$

Considerando as regras $R_{1}, R_{2}$ e $R_{3}$ da Tabela 3.6, a confiança negativa de cada regra resulta respectivamente $\mathrm{em}$ :

$$
\begin{aligned}
& \operatorname{NegRel}\left(R_{1}\right)=\frac{5}{10}=0.500 \\
& \operatorname{NegRel}\left(R_{2}\right)=\frac{9}{12}=0.750 \\
& \operatorname{NegRel}\left(R_{3}\right)=\frac{6}{13}=0.461
\end{aligned}
$$

Para a matriz de confusão da Tabela 3.7, o valor da confiança negativa da hipótese é:

$$
\operatorname{NegRel}(\mathbf{h})=\frac{5}{5+1}=0.833
$$

\section{Sensitividade e Especificidade}

Definição 3.3.4. Sensitividade: $\operatorname{Sens}(B \rightarrow H)=P(B \mid H)$

Definição 3.3.5. Especificidade: $\operatorname{Spec}(B \rightarrow H)=P(\bar{B} \mid \bar{H})$

A sensitividade de uma regra é semelhante ao recall de casos positivos usados em recuperação de informação (Information retrieval). Também conhecida como completeza, é uma medida do número (relativo) de exemplos da classe prevista em $H$ cobertos pela regra. No framework proposto por Lavrac, ela é definida como a probabilidade condicional de $B$ ser verdade dado que $H$ é verdade.

$$
\operatorname{Sens}(R)=P(B \mid H)=\frac{P(H B)}{P(H)}=\frac{f_{h b}}{f_{h}}=\frac{h b}{h}
$$

Como mencionado anteriormente, no caso de problemas de classificação binária, a sensitividade da hipótese (classificador) mede a fração de verdadeiros positivos que são 
corretamente classificados, ou seja, recall de casos positivos, dada por:

$$
\operatorname{Sens}(\mathbf{h})=\frac{T p}{T p+F n}
$$

A especificidade de uma regra é o correspondente à completeza, mas para os exemplos que não são cobertos pela regra $R$. É definida como a probabilidade condicional de $B$ ser falso dado que $H$ é falso, ou seja:

$$
\operatorname{Spec}(R)=P(\bar{B} \mid \bar{H})=\frac{P(\overline{H B})}{P(\bar{H})}=\frac{f_{\overline{h b}}}{f_{\bar{h}}}=\frac{\overline{h b}}{\bar{h}}
$$

Em problemas de classificação binária, é semelhante ao recall dos casos negativos na recuperação de informação:

$$
\operatorname{Spec}(\mathbf{h})=\frac{T n}{T n+F p}
$$

Considerando as regras $R_{1}, R_{2}$ e $R_{3}$ da Tabela 3.6, a sensitividade e a especificidade de cada regra resulta respectivamente em:

$$
\begin{array}{ll}
\operatorname{Sens}\left(R_{1}\right)=\frac{4}{9}=0.444 & \operatorname{Spec}\left(R_{1}\right)=\frac{5}{6}=0.833 \\
\operatorname{Sens}\left(R_{2}\right)=\frac{3}{6}=0.500 & \operatorname{Spec}\left(R_{2}\right)=\frac{9}{9}=1.000 \\
\operatorname{Sens}\left(R_{3}\right)=\frac{2}{9}=0.222 & \operatorname{Spec}\left(R_{3}\right)=\frac{6}{6}=1.000
\end{array}
$$

Para a matriz de confusão da Tabela 3.7, o valor da sensitividade e da especificidade da hipótese (classificador) é:

$$
\begin{aligned}
& \operatorname{Sens}(\mathbf{h})=\frac{8}{8+1}=0.889 \\
& \operatorname{Spec}(\mathbf{h})=\frac{5}{5+1}=0.833
\end{aligned}
$$




\section{Cobertura e Suporte}

Definição 3.3.6. Cobertura: $\operatorname{Cov}(B \rightarrow H)=P(B)$

Definição 3.3.7. Suporte: $\operatorname{Sup}(B \rightarrow H)=P(H B)$

A cobertura de uma regra é uma medida do número (relativo) de exemplos cobertos pela regra $R$. Como tal, é uma medida de generalidade da regra. Essa medida é definida como:

$$
\operatorname{Cov}(R)=P(B)=f_{b}=\frac{b}{n}
$$

Quanto maior a cobertura, maior o número de exemplos cobertos pelo corpo da regra $R$.

O suporte de uma regra é uma medida do número (relativo) de exemplos cobertos corretamente pela regra $R$. É definida como:

$$
\operatorname{Sup}(R)=P(H B)=f_{h b}=\frac{h b}{n}
$$

Conhecida do aprendizado de regras de associação, essa medida também é chamada de freqüência. Quanto maior o suporte, maior o número de exemplos cobertos pelo corpo e a cabeça da regra $R$. Vale a pena notar que, diferentemente das medidas anteriores, o suporte é simétrico em $H$ e $B$, ou seja,

$$
\operatorname{Sup}(R)=P(H B)=P(B H)
$$

Considerando as regras $R_{1}, R_{2}$ e $R_{3}$ da Tabela 3.6, a cobertura e o suporte de cada regra resulta respectivamente em:

$$
\begin{array}{ll}
\operatorname{Cov}\left(R_{1}\right)=\frac{5}{15}=0.333 & \operatorname{Sup}\left(R_{1}\right)=\frac{4}{15}=0.267 \\
\operatorname{Cov}\left(R_{2}\right)=\frac{3}{15}=0.200 & \operatorname{Sup}\left(R_{2}\right)=\frac{3}{15}=0.200 \\
\operatorname{Cov}\left(R_{3}\right)=\frac{2}{15}=0.133 & \operatorname{Sup}\left(R_{3}\right)=\frac{2}{15}=0.133
\end{array}
$$




\section{Novidade}

Definição 3.3.8. Novidade: $N o v(B \rightarrow H)=P(H B)-P(H) P(B)$

A medida de novidade tem a intenção de mostrar o quanto uma regra é interessante, nova $^{3}$ ou fora do comum. Essa medida foi inicialmente apresentada em (Piatetsky-Shapiro, 1991) como medida $P S(R)$ e está ilustrada na Equação 4.2, página 48. A seguir é definida uma medida de novidade utilizando a estrutura da tabela de contingência (Tabela 3.2).

Define-se uma regra $B \rightarrow H$ como nova se a probabilidade de $B$ e $H$ ocorrerem juntos não puder ser inferida pelas probabilidades de $B$ e $H$ isoladamente, isto é, $B$ e $H$ não são estatisticamente independentes.

A medida de novidade é obtida comparando o valor esperado $P(H B)$ com os valores de $P(H)$ e $P(B)$. Quanto mais o valor esperado diferir do observado, maior é a probabilidade que exista um correlação verdadeira e inesperada entre $B$ e $H$. Pode ser demonstrado que $-0,25 \leq \operatorname{Nov}(R) \leq 0,25$, e quanto maior um valor positivo mais forte é a associação entre $B$ e $H$ enquanto que, quanto maior um valor negativo, mais forte é a associação entre $B$ e $\bar{H}$. Se $N o v(R)=0$ a regra não é interessante, nova ou fora do comum.

A novidade de uma regra é então definida como:

$$
N o v(R)=P(H B)-P(H) P(B)=f_{h b}-f_{h} \cdot f_{b}=\frac{1}{n}\left(h b-\frac{h \cdot b}{n}\right)
$$

Considerando as regras $R_{1}, R_{2}$ e $R_{3}$ da Tabela 3.6, a novidade de cada regra resulta respectivamente em:

$$
\begin{aligned}
& \operatorname{Nov}\left(R_{1}\right)=\frac{4}{15}-\left(\frac{9}{15} \cdot \frac{5}{15}\right)=0.067 \\
& \operatorname{Nov}\left(R_{2}\right)=\frac{3}{15}-\left(\frac{6}{15} \cdot \frac{3}{15}\right)=0.120 \\
& \operatorname{Nov}\left(R_{3}\right)=\frac{2}{15}-\left(\frac{9}{15} \cdot \frac{2}{15}\right)=0.053
\end{aligned}
$$

\footnotetext{
${ }^{3}$ Do termo original em inglês novelty, aqui traduzido como adjetivo, no sentido de ser capaz de trazer consigo alguma novidade ou originalidade, sinônimo de original.
} 


\section{Satisfação}

Definição 3.3.9. Satisfação: $\operatorname{Sat}(B \rightarrow H)=\frac{P(\bar{H})-P(\bar{H} \mid B)}{P(\bar{H})}$.

A satisfação de uma regra é medida pelo aumento relativo na precisão entre a regra $B \rightarrow$ verdade e a regra $B \rightarrow H$. É uma medida capaz de promover um equilíbrio entre regras com diferentes condições e conclusões.

$$
\operatorname{Sat}(R)=\frac{P(\bar{H})-P(\bar{H} \mid B)}{P \bar{H}}=\frac{f_{\bar{h}}-\frac{f_{\bar{h} b}}{f_{b}}}{f_{\bar{h}}}=1-\left(\frac{\bar{h} b}{b} \cdot \frac{n}{\bar{h}}\right)
$$

É fácil mostrar que $\operatorname{Sat}(B \rightarrow H)=\frac{P(H \mid B)-P(H)}{1-P(H)}$ já que $P(\bar{H})-P(\bar{H} \mid B)=(1-P(H))-$ $(1-P(H \mid B))=P(H \mid B)-P(H)$. Pode ser observado que $S a t(R)$ está relacionada com $A c c(R)$, pois $\operatorname{Sat}(R)=1 \operatorname{sss}^{4} A c c(R)=1$. Entretanto, diferente da precisão de uma regra, a satisfação leva em conta o total da tabela de contingência e trata da associação de diferentes cabeças para um mesmo corpo de uma regra, sendo assim mais indicada para tarefas voltadas à descoberta de conhecimento.

Considerando as regras $R_{1}, R_{2}$ e $R_{3}$ da Tabela 3.6, a satisfação de cada regra resulta respectivamente em:

$$
\begin{aligned}
& \operatorname{Sat}\left(R_{1}\right)=\left(\frac{6}{15}-\frac{1}{5}\right) \div \frac{6}{15}=0.500 \\
& \operatorname{Sat}\left(R_{2}\right)=\left(\frac{9}{15}-\frac{0}{3}\right) \div \frac{9}{15}=1.000 \\
& \operatorname{Sat}\left(R_{3}\right)=\left(\frac{6}{15}-\frac{0}{2}\right) \div \frac{6}{15}=1.000
\end{aligned}
$$

A Tabela 3.8 condensa os valores das medidas genéricas de avaliação de regras obtidas para cada uma das três regras utilizadas para ilustrá-las.

\subsubsection{Medidas Relativas de Avaliação de Regras}

Na seção anterior foram apresentadas algumas medidas genéricas de regras utilizando um framework geral para descobrimento de conhecimento proposto em (Lavrac et al.,

\footnotetext{
${ }^{4}$ se e somente se.
} 


\begin{tabular}{|l|c|c|c|}
\hline Medida & $R_{1}$ & $R_{2}$ & $R_{3}$ \\
\hline$A c c(B \rightarrow H)$ & 0.800 & 1.000 & 1.000 \\
\hline $\operatorname{Err}(B \rightarrow H)$ & 0.200 & 0.000 & 0.000 \\
\hline NegRel $(B \rightarrow H)$ & 0.500 & 0.750 & 0.461 \\
\hline Sens $(B \rightarrow H)$ & 0.444 & 0.500 & 0.222 \\
\hline Spec $(B \rightarrow H)$ & 0.833 & 1.000 & 1.000 \\
\hline $\operatorname{Cov}(B \rightarrow H)$ & 0.333 & 0.200 & 0.133 \\
\hline $\operatorname{Sup}(B \rightarrow H)$ & 0.267 & 0.200 & 0.133 \\
\hline Nov $(B \rightarrow H)$ & 0.067 & 0.120 & 0.053 \\
\hline Sat $(B \rightarrow H)$ & 0.500 & 1.000 & 1.000 \\
\hline \hline
\end{tabular}

Tabela 3.8: Valores das Medidas Genéricas de Avaliação das Regras $R_{1}, R_{2}$ e $R_{3}$

1999). Nesta seção é mostrada a utilidade desse framework estabelecendo uma síntese dessas medidas. A principal inspiração para essa síntese vem da medida novidade, a qual pode ser considerada relativa já que compara o suporte da regra com o suporte esperado, sobre a suposição de independência estatística (Definição 3.3.8).

\section{Precisão Relativa}

Definição 3.3.10. Precisão relativa: $R A c c(B \rightarrow H)=P(H \mid B)-P(H)$

A precisão relativa de uma regra $R=B \rightarrow H$ é o ganho de precisão obtido em relação a uma regra padrão true $\rightarrow H$, ou seja, uma regra que avalia $B$ como verdade para todos os exemplos. Nesse caso, uma regra só interessa se melhorar a precisão da regra padrão. Essa medida relativa é definida como:

$$
R A c c(R)=P(H \mid B)-P(H)=\frac{f_{h b}}{f_{b}}-f_{h}=\frac{h b}{b}-\frac{h}{n}
$$

Outra forma de ver a precisão relativa de uma regra é como uma medida de utilidade da conexão de um corpo $B$ com uma dada cabeça $H$.

Considerando as regras $R_{1}, R_{2}$ e $R_{3}$ da Tabela 3.6, a precisão relativa de cada regra resulta respectivamente em:

$$
\begin{aligned}
& R \operatorname{Acc}\left(R_{1}\right)=\frac{4}{5}-\frac{9}{15}=0.200 \\
& \operatorname{RAcc}\left(R_{2}\right)=\frac{3}{3}-\frac{6}{15}=0.600 \\
& \operatorname{RAcc}\left(R_{3}\right)=\frac{2}{2}-\frac{9}{15}=0.400
\end{aligned}
$$


Similarmente, são definidas por Lavrac em seuframework, versões relativas de outras medidas de avaliação de regras.

\section{Confiança Negativa, Sensitividade e Especificidade Relativas}

Definição 3.3.11. Confiança negativa relativa: $R N$ egRel $(B \rightarrow H)=P(\bar{H} \mid \bar{B})-P(\bar{H})$

Definição 3.3.12. Sensitividade relativa: $\operatorname{RSens}(B \rightarrow H)=P(B \mid H)-P(B)$

Definição 3.3.13. Especificidade relativa: $R \operatorname{Spec}(B \rightarrow H)=P(\bar{B} \mid \bar{H})-P(\bar{B})$

A confiança negativa relativa é o análogo a precisão relativa para os exemplos que não são cobertos pela regra. Nesse caso, a regra padrão é falso $\rightarrow \bar{H}$.

$$
R N e g R e l(R)=P(\bar{H} \mid \bar{B})-P(\bar{H})=\frac{f_{\overline{h b}}}{f_{\bar{b}}}-f_{\bar{h}}=\frac{\overline{h b}}{\bar{b}}-\frac{\bar{h}}{n}
$$

A sensitividade relativa mede o ganho de sensitividade obtido em relação à sensitividade de uma regra padrão $B \rightarrow$ verdade, ou seja, uma regra que avalia $H$ como verdade para todos os exemplos.

$$
R \operatorname{Sens}(R)=P(B \mid H)-P(B)=\frac{f_{h b}}{f_{h}}-f_{b}=\frac{h b}{h}-\frac{b}{n}
$$

A especificidade relativa é o análogo a sensitividade relativa para os exemplos que não são cobertos pela regra. Nesse caso, a regra padrão é $\bar{B} \rightarrow$ falso.

$$
R \operatorname{Spec}(R)=P(\bar{B} \mid \bar{H})-P(\bar{B})=\frac{f_{\overline{h b}}}{f_{\bar{h}}}-f_{\bar{b}}=\frac{\overline{h b}}{\bar{h}}-\frac{\bar{b}}{n}
$$

Deve ser observado que a visão utilizada na construção dessas medidas é a mesma empregada pelo algoritmo CN2 (Clark and Niblett, 1989), que primeiro desenvolve o corpo da regra e depois encontra uma cabeça apropriada para esse corpo.

Considerando as regras $R_{1}, R_{2}$ e $R_{3}$ da Tabela 3.6, a confiança negativa, sensitividade 
e especificidade relativas de cada regra resulta respectivamente em:

$$
\begin{aligned}
& R N e g \operatorname{Rel}\left(R_{1}\right)=\frac{5}{10}-\frac{6}{15}=0.100 \\
& R N e g \operatorname{Rel}\left(R_{2}\right)=\frac{9}{12}-\frac{9}{15}=0.150 \\
& R N e g \operatorname{Rel}\left(R_{3}\right)=\frac{6}{13}-\frac{6}{15}=0.061 \\
& \operatorname{RSens}\left(R_{1}\right)=\frac{4}{9}-\frac{5}{15}=0.111 \\
& \operatorname{RSens}\left(R_{2}\right)=\frac{3}{6}-\frac{3}{15}=0.300 \\
& \operatorname{RSens}\left(R_{3}\right)=\frac{2}{9}-\frac{2}{15}=0.089 \\
& R \operatorname{Spec}\left(R_{1}\right)=\frac{5}{6}-\frac{10}{15}=0.167 \\
& R \operatorname{Spec}\left(R_{2}\right)=\frac{9}{9}-\frac{12}{15}=0.200 \\
& R \operatorname{Spec}\left(R_{3}\right)=\frac{6}{6}-\frac{13}{15}=0.133
\end{aligned}
$$

O ponto importante referente a medidas relativas é que elas fornecem mais informações sobre a utilidade de uma regra do que as informações fornecidas pelas medidas absolutas. Por exemplo, se em uma tarefa de predição a precisão de uma regra é menor que a freqüência relativa da classe que a regra prediz, então a regra tem uma performance ruim, independente da sua precisão absoluta.

Existe, no entanto, um problema com a precisão relativa: é fácil obter uma alta precisão relativa para regras específicas, ou seja, regras com baixa generalidade (generalidade $=P(B)$ ). Para contornar essa dificuldade, uma variante é proposta em (Lavrac et al., 1999), na qual é atribuído um peso para cada medida. Este peso promove um balanceamento $^{5}$ entre a generalidade e a relatividade dessas medidas, como descrito a seguir.

\footnotetext{
${ }^{5}$ Neste trabalho, balanceamento é a tradução adotada para o termo em inglês "trade off".
} 


\subsubsection{Medidas Relativas de Regras com Peso}

\section{Precisão relativa com peso}

Definição 3.3.14. Precisão relativa com peso:

$$
W R A c c(R)=P(B)(P(H \mid B)-P(H))=f_{b}\left(\frac{f_{h b}}{f_{b}}-f_{h}\right)=\frac{b}{n}\left(\frac{h b}{b}-\frac{h}{n}\right)
$$

A precisão relativa com peso faz um balanceamento entre generalidade e precisão relativa.

É conhecida na literatura como uma medida de ganho, usada para avaliar a utilidade de um literal $L$ considerado para estender o corpo $B$ de uma regra: $\frac{P(B L)}{P(B)}(P(H \mid B L)-$ $P(H \mid B))$.

Considerando as regras $R_{1}, R_{2}$ e $R_{3}$ da Tabela 3.6, a precisão relativa com peso de cada regra resulta respectivamente em:

$$
\begin{aligned}
& W R A c c\left(R_{1}\right)=\frac{5}{15} \cdot\left(\frac{4}{5}-\frac{9}{15}\right)=0.067 \\
& W R A c c\left(R_{2}\right)=\frac{3}{15} \cdot\left(\frac{3}{3}-\frac{6}{15}\right)=0.120 \\
& W R A c c\left(R_{3}\right)=\frac{2}{15} \cdot\left(\frac{2}{2}-\frac{9}{15}\right)=0.053
\end{aligned}
$$

O resultado a seguir provê uma contribuição significante para o entendimento de medidas de avaliação de regra.

Teorema 3.3.1. $W R A c c(R)=N o v(R)$

Prova.

$$
\begin{aligned}
W R A c c(B \rightarrow H) & =P(B)(P(H \mid B)-P(H)) \\
& =(P(B) P(H \mid B)-P(B) P(H)) \\
& =P(H B)-P(H) P(B) \\
& =N o v(B \rightarrow H) .
\end{aligned}
$$

Este teorema tem as seguintes implicações:

1. Regras com alta precisão relativa com peso também têm alta novidade e vice-versa. 
2. Alta novidade é alcançada pelo balanceamento entre a generalidade e a precisão da regra, obtido em comparação com a regra trivial true $\rightarrow H$. Isto também significa que ter uma alta precisão relativa não é o bastante para considerar uma regra como sendo interessante, já que a regra necessita também ser suficientemente genérica.

Esse teorema é importante, pois estabelece um elo entre as medidas de avaliação de regras.

Deve ser observado que a precisão relativa com peso é uma medida fundamental de avaliação de regras, pois fornece um balanceamento entre precisão e outras medidas supervisionadas, como visto a seguir.

\section{Confiança Negativa, Sensitividade e Especificidade Relativas com Peso}

Definição 3.3.15. Confiança negativa relativa com peso:

$$
W R N e g R e l(R)=P(\bar{B})(P(\bar{H} \mid \bar{B})-P(\bar{H}))=f_{\bar{b}}\left(\frac{f_{\overline{h b}}}{f_{\bar{b}}}-f_{\bar{h}}\right)=\frac{\bar{b}}{n}\left(\frac{\overline{h b}}{\bar{b}}-\frac{\bar{h}}{n}\right)
$$

O uso do peso $P(\bar{B})$ é motivado pelo fato da maioria das regras muito gerais terem uma confiança negativa alta.

Definição 3.3.16. Sensitividade relativa com peso:

$$
W R \operatorname{Sens}(R)=P(H)(P(B \mid H)-P(B))=f_{h}\left(\frac{f_{h b}}{f_{h}}-f_{b}\right)=\frac{h}{n}\left(\frac{h b}{h}-\frac{b}{n}\right)
$$

Definição 3.3.17. Especificidade relativa com peso:

$$
W R \operatorname{Spec}(R)=P(\bar{H})(P(\bar{B} \mid \bar{H})-P(\bar{B}))=f_{\bar{h}}\left(\frac{f_{\overline{h b}}}{f_{\bar{h}}}-f_{\bar{b}}\right)=\frac{\bar{h}}{n}\left(\frac{\overline{h b}}{\bar{h}}-\frac{\bar{b}}{n}\right)
$$

Como nos casos anteriores, os pesos têm como objetivo prevenir soluções triviais. Considerando as regras $R_{1}, R_{2}$ e $R_{3}$ da Tabela 3.6, a confiança negativa, sensitividade e especificidade relativas com peso de cada regra resultam respectivamente em: 


$$
\begin{aligned}
& W R N \text { egRel }\left(R_{1}\right)=\frac{10}{15} \cdot\left(\frac{5}{10}-\frac{6}{15}\right)=0.067 \\
& W R N \text { egRel }\left(R_{2}\right)=\frac{12}{15} \cdot\left(\frac{9}{12}-\frac{9}{15}\right)=0.120 \\
& W R N \text { egRel }\left(R_{3}\right)=\frac{13}{15} \cdot\left(\frac{6}{13}-\frac{6}{15}\right)=0.053
\end{aligned}
$$

$$
\begin{aligned}
& W R \operatorname{Rens}\left(R_{1}\right)=\frac{9}{15} \cdot\left(\frac{4}{9}-\frac{5}{15}\right)=0.067 \\
& W R \operatorname{RSens}\left(R_{2}\right)=\frac{6}{15} \cdot\left(\frac{3}{6}-\frac{3}{15}\right)=0.120 \\
& W R \operatorname{Rens}\left(R_{3}\right)=\frac{9}{15} \cdot\left(\frac{2}{9}-\frac{2}{15}\right)=0.053
\end{aligned}
$$

$$
\begin{aligned}
& W R \operatorname{Rpec}\left(R_{1}\right)=\frac{6}{15} \cdot\left(\frac{5}{6}-\frac{10}{15}\right)=0.067 \\
& W R \operatorname{Spec}\left(R_{2}\right)=\frac{9}{15} \cdot\left(\frac{9}{9}-\frac{12}{15}\right)=0.120 \\
& W R \operatorname{Spec}\left(R_{3}\right)=\frac{6}{15} \cdot\left(\frac{6}{6}-\frac{13}{15}\right)=0.053
\end{aligned}
$$

Como pode ser observado, essas medidas são as mesmas para cada uma das três regras. Na realidade, é possível estabelecer uma relação entre as quatro medidas padrão de avaliação de regras supervisionadas por meio do relacionamento dessas com suas variantes com peso.

Teorema 3.3.2. $W R A c c(R)=W R \operatorname{Sens}(R)=W R \operatorname{Spec}(R)=W R N \operatorname{Reg} \operatorname{Rel}(R)$

Prova.

$W R A c c(B \rightarrow H)=P(B)(P(H \mid B)-P(H))$ 


$$
\begin{aligned}
& =P(H B)-P(H) P(B) \\
& =P(H)(P(B \mid H)-P(B)) \\
& =W \operatorname{RSens}(B \rightarrow H) .
\end{aligned}
$$

$$
\begin{aligned}
W R A c c(B \rightarrow H) & =P(B)(P(H \mid B)-P(H)) \\
& =P(H B)-P(H) P(B) \\
& =(1-P(\bar{H} B)-P(H \bar{B})-P(\overline{H B}))-(1-P(\bar{B}))(1-P(\bar{H})) \\
& =(1-P(\bar{H})-P(\bar{B})+P(\overline{H B}))-(1-P(\bar{H})-P(\bar{B})+P(\bar{H}) P(\bar{B})) \\
& =P(\overline{H B})-P(\bar{H}) P(\bar{B}) \\
& =P(\bar{H})(P(\bar{B} \mid \bar{H})-P(\bar{B})) \\
& =W R \operatorname{Spec}(B \rightarrow H) .
\end{aligned}
$$

$$
\begin{aligned}
W R A c c(B \rightarrow H) & =W R \operatorname{Spec}(B \rightarrow H) \\
& =P(\bar{H})(P(\bar{B} \mid \bar{H})-P(\bar{B})) \\
& =P(\bar{B})(P(\bar{H} \mid \bar{B})-P(\bar{H})) \\
& =W R N e g \operatorname{Rel}(B \rightarrow H)
\end{aligned}
$$

A Tabela 3.9 condensa os valores das medidas relativas de avaliação de regras, com e sem peso, obtidas para cada uma das três regras utilizadas para ilustrá-las.

\begin{tabular}{|l|c|c|c|}
\hline Medida & $R_{1}$ & $R_{2}$ & $R_{3}$ \\
\hline$R A c c(B \rightarrow H)$ & 0.200 & 0.600 & 0.400 \\
\hline$R N e g R e l(B \rightarrow H)$ & 0.100 & 0.150 & 0.061 \\
\hline$R \operatorname{Sens}(B \rightarrow H)$ & 0.111 & 0.300 & 0.089 \\
\hline$R \operatorname{Spec}(B \rightarrow H)$ & 0.167 & 0.200 & 0.133 \\
\hline$W R A c c(B \rightarrow H)^{a}$ & 0.067 & 0.120 & 0.053 \\
\hline \hline
\end{tabular}

${ }^{a}$ Medidas Relativas com Pesos $W R A c c(B \rightarrow H)=W \operatorname{RNegRel}(B \rightarrow H)=$ $W R \operatorname{Sens}(B \rightarrow H)=W R \operatorname{Spec}(B \rightarrow H)$.

Tabela 3.9: Valores das Medidas Relativas de Avaliação das Regras $R_{1}, R_{2}$ e $R_{3}$

\subsection{Considerações Finais}

As medidas de avaliação de regras são importantes para que o usuário possa focalizar sua atenção sob aquelas regras melhor sustentadas pelos dados, ou seja, focalizar sua 
atenção sob as regras de maior qualidade.

No entanto, mesmo aquelas regras que apresentam um bom desempenho para uma ou várias medidas diferentes, podem revelar um conhecimento óbvio ou irrelevante para o usuário. A análise quantitativa de regras tem o intuito de selecionar aquelas regras que possam trazer algum conhecimento surpreendente ou inesperado para o usuário.

No capítulo seguinte são apresentadas algumas medidas objetivas de interessabilidade, que podem ser utilizadas para efetuar a análise quantitativa de regras. 


\section{Capítulo 4}

\section{Interessabilidade de Regras}

\subsection{Do termo Interessabilidade}

Interessabilidade ${ }^{1}$ é a tradução da palavra em inglês interestingness, já aceita e difundida pela comunidade científica (Pistory, 1998; Horst and Monard, 2000), que será adotada nesse trabalho. Entretanto, vale ressaltar a inapropriação do termo ao sentido pretendido: morfologicamente, interessabilidade só pode significar "qualidade do que é interessável", ou seja, "do que pode ser interessado".

A rigor, portanto, uma medida de interessabilidade de uma regra deveria, na verdade, referir-se a quanto uma regra "pode ser interessada", obviamente uma interpretação absurda, mas a única possível. Para significar "qualidade do que é interessante", como pretenderam ao idealizadores de "interestingness", um neologismo português (que guarda uma analogia perfeita com o termo original inglês, mas simplesmente parece estranho demais para ser aceito) morfologicamente plausível é interessância (relevante | relevância, conveniente | conveniência, etc.). É curioso notar, por fim, que ambas as línguas já possuem um substantivo atestado (interest | interesse) que substituiria perfeitamente interestingness, mas que parece ter sido preterido por ser usado com mais freqüência em outras acepções.

Em resumo, o termo interessabilidade, apesar de morfologicamente possível, não é semanticalmente aceitável mas acabou por se estabelecer pelo uso.

\footnotetext{
${ }^{1}$ A reflexão aqui apresentada foi elaborada por Jorge M. Pelizzoni, mestre pelo Núcleo Interinstitucional de Lingüística Computacional - NILC - ICMC/USP
} 


\subsection{Considerações Iniciais}

O conhecimento adquirido por sistemas de Aprendizado de Máquina indutivo pode ser avaliado pelo usuário de diferentes maneiras. Quando esse conhecimento está representado por regras, uma maneira mais simples é a própria interpretação da regra, isto é, analisar as informações nela contida. Isso pode até ser suficiente numa análise inicial, no entanto, há muitas outras informações relacionadas às regras que podem ser obtidas, por exemplo, utilizando as medidas de avaliação abordadas no Capítulo 3.

A análise de regras utilizando medidas de avaliação tem a intenção de apontar quais as regras melhor sustentadas em função dessas medidas. No entanto, o conhecimento descrito por essas regras pode ser óbvio ou irrelevante, mesmo quando elas apresentam um bom desempenho para alguma medida de avaliação.

Seguindo os aspectos e princípios encontrados na literatura, neste capítulo são apresentadas algumas medidas de interessabilidade de regras que podem ser calculadas de forma independente em relação ao domínio de aplicação. Deve ser ressaltado que essas medidas já foram anteriormente estudas por Paulo Sérgio Horst e que este capítulo está fortemente baseado em seu trabalho (Horst, 1999).

\subsection{Aspectos de Interessabilidade de Regras}

Medidas de interessabilidade podem selecionar regras tentando capturar o quanto esse conhecimento é interessante (ou inesperado), segundo critérios como utilidade e potencialidade de uso. Dois aspectos de medidas de interessabilidade de regras são encontrados na literatura (Silberschatz and Tuzhilin, 1996) e (Freitas, 1998a):

- Aspecto Subjetivo ou orientado ao usuário - a interessabilidade está estritamente relacionada às impressões gerais do usuário sobre uma regra. Medidas que consideram esse aspecto são conhecidas como medidas subjetivas de interessabilidade.

- Aspecto Objetivo ou orientado a dados - a interessabilidade está estritamente relacionada aos dados da regra, assim, ela é autônoma e pode ser computada. Medidas que consideram esse aspecto são conhecidas como medidas objetivas de interessabilidade. 


\subsubsection{Medidas Subjetivas de Interessabilidade}

Do ponto de vista subjetivo, (Silberschatz and Tuzhilin, 1995) propõe duas categorias de classificação para que o conhecimento adquirido sob a forma de regras seja considerado interessante. São elas:

- Acionabilidade: o usuário pode tirar alguma vantagem ao utilizar esse conhecimento.

- Inesperabilidade: o conhecimento adquirido é inesperado, ou seja, causa "surpresa" ao usuário.

A acionabilidade é uma medida importante, principalmente porque os usuários, freqüentemente, estão interessados nas vantagens que podem conseguir tomando ações com o novo conhecimento adquirido. No entanto, não é fácil decidir quais regras são acionáveis, sendo uma maneira a verificação de seu uso na prática.

A inesperabilidade, como medida subjetiva, está estritamente relacionada às crenças ou impressões gerais do usuário.

As crenças podem ser classificadas em dois tipos: crenças rígidas e crenças flexíveis. As crenças rígidas são as restrições que não podem ser mudadas com novas evidências, enquanto que as crenças flexíveis são aquelas passíveis de alguma mudança.

Essas duas categorias de classificação de medidas subjetivas não são mutuamente exclusivas e podem dar lugar à seguinte classificação:

1. regras que são tanto inesperadas quanto acionáveis;

2. regras que são inesperadas mas não acionáveis;

3. regras que são acionáveis mas esperadas.

As regras da primeira e segunda categorias podem ser obtidas pela busca de regras inesperadas, enquanto que as da terceira categoria podem ser obtidas pela busca de regras que estejam em conformidade com conceitos prévios do usuário. 


\subsubsection{Medidas Objetivas de Interessabilidade}

Medidas objetivas estão relacionadas, em geral, às propriedades de surpresa (inesperabilidade), potencialidade de uso e novidade (Freitas, 1998b). Do ponto de vista objetivo, a interessabilidade de uma regra é dada em termos de sua estrutura e dos dados envolvidos no processo de descoberta do conhecimento.

Num processo de análise de regras, a abordagem objetiva pode ser usada como uma espécie de filtro para selecionar regras potencialmente interessantes e, posteriormente, submetê-las a uma avaliação subjetiva, determinando assim qual conhecimento é realmente interessante para o usuário.

\subsection{Princípios para Interessabilidade}

Conforme apresentado na Seção 3.2.1, página 20, regras preditivas proposicionais podem ser representadas na forma $B \rightarrow H$, isto é, if $B$ then $H$, mais especificamente:

$$
\text { if } \underbrace{\operatorname{cond}_{1} \text { and } \operatorname{cond}_{2} \ldots \text { and } \operatorname{cond}_{m}}_{B} \text { then } \underbrace{\text { classe } C_{v}}_{H}
$$

onde:

$C_{v}$ representa um dos possíveis $j$ valores da classe, isto é, $C_{v}=\left\{C_{1}, C_{2}, \ldots, C_{N C l}\right\}$; $\operatorname{cond}_{g}, g=1 \ldots m$, é uma tripla da forma $<A t$ op $V a l>$ onde:

At é um atributo;

$o p$ é um operador relacional que pertence ao conjunto $\{<\rangle,, \leq, \geq,=\}$;

Val é um dos possíveis valores do domínio de $A t$.

Em geral, se o atributo $A t$ for contínuo, então op pertence ao conjunto $\{<\rangle,, \leq, \geq\}$, enquanto que se o atributo At for discreto, op é o operador "=".

Considerando-se regras preditivas proposicionais, foram propostos por (PiatetskyShapiro, 1991) três princípios básicos desejáveis para uma medida de Interessabilidade de Regras $(I R)$. Ele conjecturou que todas as medidas satisfazendo esses princípios atribuiriam alto valor para regras fortes e baixo valor para regras fracas. Uma regra forte 
pode ser definida como uma descrição de uma regularidade, com alta confiança, para um grande número de exemplos. Uma regra fraca representa a descrição de uma regularidade, com alta confiança, para um pequeno número de exemplos (Suzuki and Kodratoff, 1998).

Os princípios são os seguintes:

1. $I R=0$ se $|B \cap H|=\frac{|B||H|}{n}$. Se $B$ e $H$ são estatisticamente independentes, a regra não é interessante.

2. IR aumenta monotonicamente com $|B \cap H|$ quando outros parâmetros permanecem fixos;

3. IR diminui monotonicamente com $|B|$ ou $|H|$ quando outros parâmetros permanecem fixos;

Esses princípios apresentam algumas implicações interessantes. Primeiro, sobre o princípio $1, I R>0$ se $|B \cap H|>\frac{|B||H|}{n}$ (indica que $B$ está positivamente correlacionado a $H$ ) e $I R<0$ se $|B \cap H|<\frac{|B||H|}{n}$ (indica que $B$ está negativamente correlacionado a $H)$. A esse respeito, $I R$ é similar ao coeficiente de correlação entre $B$ e $H$. Segundo, pelo fato de se ter sempre $|B \cap H| \leq|B| \leq|H|$ os princípios 2 e 3 implicam que $I R$ tem um máximo local quando $|B \cap H|=|B|$ ou $|B|=|H|$ e um máximo global quando $|B \cap H|=|B|=|H|$.

Esses princípios podem ser estendidos para outros parâmetros da regra como, por exemplo, complexidade da regra ( $I R$ diminui monotonicamente) ou tamanho do conjunto de exemplos ( $I R$ aumenta monotonicamente).

Além dos três princípios já citados, outros podem ser encontrados na literatura. Um quarto princípio foi sugerido por (Major and Mangano, 1993) no qual $I R$ aumenta monotonicamente com $|B|$ (cobertura da regra), dado um fator de confiança maior que um fator de confiança padrão (por exemplo, a probabilidade inicial das classes).

\subsection{Algumas Medidas Objetivas de Interessabilidade de Regras}

Várias medidas de interessabilidade de regras podem ser encontradas na literatura (Hilderman and Hamilton, 1999), a maior parte utiliza a abordagem subjetiva. No entanto, 
as medidas mostradas nesta seção utilizam apenas a abordagem objetiva, ou seja, podem ser computadas a partir dos dados relacionados a cada regra e podem ser calculadas de forma independente em relação ao domínio de aplicação. Usualmente, quanto maior o valor apresentado por essas medidas, maior será a interessabilidade que a regra pode apresentar.

\subsubsection{Medida $P S$}

Uma medida introduzida por (Piatetsky-Shapiro, 1991) que satisfaz os três princípios básicos de interessabilidade é definida como

$$
P S(R)=h b-\frac{h \cdot b}{n}
$$

A medida $P S(R)$ considera a questão do desbalanceamento da distribuição das classes favorecendo regras que predizem a classe minoritária. Uma distribuição de classes está desbalanceada se os exemplos de uma classe são muito mais freqüentes, ou muito mais raros, que exemplos de outras classes.

Entretanto, essa medida tem a propriedade indesejável de ser simétrica com relação ao corpo $B$ e a cabeça $H$ da regra. Algumas modificações podem ser feitas a fim de incorporar outros critérios, tais como interessabilidade dos atributos, custo de classificação incorreta e tamanho do disjunto. Esses três critérios são apresentados em (Freitas, 1998a) e têm o efeito positivo de torná-la assimétrica.

A medida $P S(R)$ também pode ser vista no framework de Lavrac (Lavrac et al., 1999) no qual é tratada como medida de novidade da regra - Definição 3.3.8 — ou seja, $\operatorname{nov}(R)=\frac{1}{n} \cdot P S(R)$.

\subsubsection{Grau de Surpresa de Pequenos Disjuntos}

Pequenos disjuntos são regras que cobrem um número pequeno de exemplos. Saber se uma regra é ou não um pequeno disjunto depende de algum critério específico relacionado com o número de exemplos cobertos pela regra (tal como um threshold ou outro critério mais flexível).

Em geral, os pequenos disjuntos são indesejáveis, porque apresentam pouca generali- 
dade e são propensos ao erro. Isso leva alguns algoritmos a utilizar um bias que favorece a descoberta de grandes disjuntos. No entanto, esses pequenos disjuntos tem o potencial para capturar conhecimento inesperado dos dados. (Freitas, 1998b) menciona o trabalho de (Provost and Aronis, 1996) que relata uma aplicação na qual um pequeno disjunto descoberto por um algoritmo revelou um conhecimento verdadeiramente interessante que levou a novas e importantes pesquisas no domínio em que foi aplicado.

A questão dos pequenos disjuntos tem sido tratada principalmente sob o ponto de vista da precisão de classificação. Sob o ponto de vista do grau de surpresa (ou mais genericamente de interessabilidade) do conhecimento descoberto, (Freitas, 1998b) apresenta algumas sugestões, mostradas nesta seção, que podem ser utilizadas para uma medida do grau de surpresa de pequenos disjuntos.

A idéia geral dessa medida é que ela deve considerar um pequeno disjunto como conhecimento surpresa quando esse disjunto prediz uma classe diferente das classes preditas pelas suas generalizações mínimas GM. Um pequeno disjunto terá tantas generalizações quantas forem as suas condições, isto é, cada uma das $g$ generalizações $(g=1 \cdots m)$ está associada com uma das $m$ condições cond $_{g}$ do disjunto original.

Usualmente, a maneira de se obter as generalizações depende do tipo dos atributos. Se o atributo $A t$ presente em cond $_{g}$ é discreto, então a $g$-ésima generalização é obtida ao remover $\operatorname{cond}_{g}$. Por outro lado, se $A t$ for contínuo a $g$-ésima generalização pode ser obtida de duas formas.

1. remover cond $_{g}$ do disjunto (semelhante ao caso de $A t$ ser discreto);

2. adicionar um pequeno valor $\alpha$ a $V a l$ em $\operatorname{cond}_{g}$ quando op pertence a $\{<, \leq\}$ ou subtrair $\alpha$ de $V$ al em cond $_{g}$, quando op pertence a $\{>, \geq\}$.

O valor de $\alpha$ deve ser especificado pelo usuário. No entanto, para casos nos quais seja necessário definir diferentes valores de $\alpha$ para vários atributos contínuos, uma alternativa é utilizar um valor relativo de $\alpha$ (como, por exemplo, um valor percentual) ou aplicar outros métodos (como, por exemplo, uma abordagem baseada em percentil). A escolha do valor de $\alpha$ e da maneira de lidar com condições que têm atributos contínuos são dependentes do domínio.

Uma maneira de medir objetivamente o grau de surpresa de pequenos disjuntos que foram descobertos por um sistema de aprendizado por exemplos utilizando a remoção de 
cond $_{g}$ para obter as $m$ geneneralizações, pode ser definido da maneira descrita a seguir. Seja a regra (pequeno disjunto):

if $\operatorname{cond}_{1}$ and $\operatorname{cond}_{2}$ and $\operatorname{cond}_{3}$ and $\cdots \operatorname{cond}_{m}$ then classe $C_{v}$

As possíveis generalizações são:

1 if $\operatorname{cond}_{2}$ and $\operatorname{cond}_{3}$ and $\cdots \operatorname{cond}_{m}$ then classe $y_{1}$

2 if $\operatorname{cond}_{1}$ and $\operatorname{cond}_{3}$ and $\cdots \operatorname{cond}_{m}$ then classe $y_{2}$

3 if $\operatorname{cond}_{1}$ and $\operatorname{cond}_{2}$ and $\cdots \operatorname{cond}_{m}$ then classe $y_{3}$

$m$ if $\operatorname{cond}_{1}$ and $\operatorname{cond}_{2}$ and $\cdots \operatorname{cond}_{m-1}$ then classe $y_{m}$

onde $y_{g}$ é a classe predita pela $g$-ésima generalização da regra. A classe $y_{g} \in\left\{C_{1}, C_{2}, \cdots C_{N C l}\right\}$ é, geralmente, a classe de maior freqüência nos exemplos cobertos pela generalização $g$. A classe $C_{v}$ do pequeno disjunto é comparada com cada $y_{g}, g=1 \cdots m$, contando o número de vezes que $y_{g}$ é diferente de $C_{v}$, isto é:

$$
\operatorname{SurpDisj}(R)=\sum_{g=1}^{m} \text { diferente }\left(y_{g}, C_{v}\right)
$$

onde

$$
\text { diferente }= \begin{cases}1 & \text { se } y_{g} \neq C_{v} \\ 0 & \text { caso contrário }\end{cases}
$$

O resultado é um número inteiro no intervalo $0 \cdots m$, o qual define o grau de surpresa do pequeno disjunto. O valor de $\operatorname{SurpDisj}(R)$ é influenciado pelo número de condições $m$ no disjunto, portanto, para evitar confusão entre medidas de complexidade sintática e medidas do grau de surpresa de disjuntos, pode ser definida a seguinte medida normalizada:

$$
\operatorname{SurpDisj}_{\text {norm }}(R)=\frac{\operatorname{SurpDisj}(R)}{m}
$$


$\operatorname{SurpDisj}_{\text {norm }}(R)$ assume valores no intervalo $0 \cdot \cdot 1$ e quanto maior esse valor mais surpresa o pequeno disjunto apresenta. No entanto, essa medida normalizada tem um bias que se direciona para regras com poucas condições, assim, provavelmente terá dificuldades para assegurar um alto valor normalizado para regras com muitas condições.

A fim de exemplificar o uso das mínimas generalizações para determinar o grau de surpresa de um pequeno disjunto, será considerada a segunda regra da Tabela 3.4:

$$
\text { if } \text { outlook }=\text { sunny and humidity } \leq 78 \text { then class }=\text { go }
$$

As possíveis generalizações dessa regra são:

$$
\begin{aligned}
& \text { if outlook }=\text { sunny then class }=d_{o n t} g o \\
& \text { if humidity } \leq 78 \text { then class }=g o
\end{aligned}
$$

A forma de determinar a classe predita por cada generalização deve ser a mesma utilizada pelo algoritmo que gerou o pequeno disjunto. Tipicamente, assume-se a classe com maior freqüência relativa entre os exemplos cobertos pela generalização. Dessa forma, considerando o conjunto de exemplos na Tabela 3.5, a classe predita para primeira generalização é "dont_go" e para a segunda é "go".

Contando o número de classes preditas pelas duas generalizações mínimas que diferem da classe predita pelo pequeno disjunto o resultado é 1 , ou seja $\operatorname{Disj} \operatorname{Surp}(R)=1$. Esse resultado é aplicado a Equação 4.4 para obter o grau de surpresa normalizado.

$$
\begin{aligned}
\operatorname{SurpDisj}_{\text {norm }}(R) & =\frac{1}{2} \\
& =0.5
\end{aligned}
$$

\subsubsection{Grau de Surpresa dos Atributos Individuais da Regra}

Várias das medidas do grau de surpresa (ou mais genericamente, medidas de interessabilidade) usualmente consideram o corpo de uma regra como um todo, sem levar em conta individualmente os atributos que ocorrem no corpo da regra. Algumas idéias baseadas na teoria da informação foram sugeridas no excelente trabalho de (Freitas, 1998b) para serem utilizadas em uma medida do grau de surpresa de regras que considera individualmente os atributos que ocorrem no corpo da regra. 
Primeiramente é calculado o ganho de informação definido como $G \operatorname{Inf} o\left(A t_{p}\right)$ de cada atributo preditivo $A t_{p}$ presente no corpo da regra.

$$
\operatorname{Surp} \operatorname{Atr}(R)=\frac{1}{\sum_{p=1}^{m} \frac{\operatorname{GInfo(At_{p})}}{m}}
$$

na qual

$$
\operatorname{GInfo}\left(A t_{p}\right)=\operatorname{Info}(C)-\operatorname{Info}\left(C \mid A t_{p}\right)
$$

com

$$
\begin{gathered}
\text { Info(C)=- } \sum_{v=1}^{N C l} \operatorname{Pr}\left(C_{v}\right) \log \operatorname{Pr}\left(C_{v}\right) \\
\text { Info }\left(C \mid A t_{p}\right)=\sum_{q=1}^{N C l p} \operatorname{Pr}\left(A t_{p q}\right)\left(-\sum_{v=1}^{N C l} \operatorname{Pr}\left(C_{v} \mid A t_{p q}\right) \log \operatorname{Pr}\left(C_{v} \mid A t_{p q}\right)\right)
\end{gathered}
$$

onde:

In $f o(C)$ é a informação da classe;

Info $\left(C \mid A t_{p}\right)$ é a informação do atributo classe $C$ dado o atributo $A t_{p}$;

$A t_{p q}$ denota o $q$-ésimo valor do atributo $A t_{p}$;

$C_{v}$ denota o $v$-ésimo valor da classe $C$;

$\mathrm{NCl}$ é o número de classes;

$N C l p$ representa o número de valores possíveis do atributo $A t_{p}$;

todos os log estão na base 2 .

As probabilidades $\operatorname{Pr}\left(C_{v}\right), \operatorname{Pr}\left(A t_{p q}\right)$ e $\operatorname{Pr}\left(C_{v} \mid A t_{p q}\right)$ podem ser obtidas aproximando-as das respectivas freqüências relativas.

Atributos com alto ganho de informação geralmente são bons preditores de classe quando considerados individualmente, no entanto, do ponto de vista da interessabilidade de regras existe a possibilidade que o usuário já conheceça quais os atributos que individualmente são melhores preditores e assim as regras contendo esses atributos tendem a ter baixo grau de surpresa para esse usuário. De outro lado estão os atributos com 
baixo ganho de informação, que muitas vezes são vistos como sem interesse pelo usuário e irrelevantes para classificação quando considerados individualmente.

A interação dos atributos pode tornar um atributo individualmente irrelevante em um atributo relevante, e esse fenômeno está intuitivamente associado com o grau de surpresa de uma regra. Considerando que outros fatores (tais como precisão da predição e cobertura) permaneçam iguais, pode-se argumentar que uma regra cujo corpo contém atributos com baixo ganho de informação pode ser apresentada como mais surpreendente que regras cujo corpo contém atributos com alto ganho de informação. Essa idéia pode ser expressa matematicamente ao definir $\operatorname{Surp} A \operatorname{tr}(R)$ como:

$$
\operatorname{Surp} A \operatorname{tr}(R)=\frac{1}{\sum_{p=1}^{m} \frac{\operatorname{GInfo(At_{p})}}{m}}
$$

A fim de exemplificar o uso do ganho de informação de atributos para determinar o grau de surpresa de uma regra, será considerada a quarta regra relacionada na Tabela 3.4, página 25, ilustrada a seguir:

$$
\text { if } \text { outlook }=\text { rain and windy }=\text { yes then class }=d o n t_{-} g o
$$

Considerando o conjunto de exemplos da Tabela 3.5, o ganho de informação do atributo "outlook", obtido pela Equação 4.6 é:

$$
\begin{aligned}
G I n f o(\text { outlook }) & =\operatorname{Info}(C)-\operatorname{Info}(\text { C|outlook }) \\
& =0.9709-0.8879 \\
& =0.083
\end{aligned}
$$

com

$$
\begin{aligned}
\operatorname{Info}(C) & =-\sum_{v=1}^{N C l} \operatorname{Pr}\left(C_{v}\right) \log \operatorname{Pr}\left(C_{v}\right) \\
& =-(-0.4421+-0.5288) \\
& =0.9709
\end{aligned}
$$




$$
\begin{aligned}
\text { Info }(C \mid \text { outlook }) & =\sum_{q=1}^{N C l p} \operatorname{Pr}\left(A t_{p q}\right)\left(-\sum_{v=1}^{N C l} \operatorname{Pr}\left(C_{v} \mid A t_{p q}\right) \log \operatorname{Pr}\left(C_{v} \mid A t_{p q}\right)\right) \\
& =0.3333(-(-0.9709))+0.3333(-(-0.7219))+0.3333(-(-0.9709)) \\
& =0.3236+0.2406+0.3236 \\
& =0.8879
\end{aligned}
$$

O ganho de informação do atributo "windy" é obtido da mesma forma:

$$
\text { GInfo(windy) }=0.035
$$

Para calcular o grau de surpresa da regra $R_{1}$ considerando seus atributos "outlook" e "windy" utiliza-se a Equação 4.9 que resulta em

$$
\begin{aligned}
\operatorname{SurpAtr}\left(R_{1}\right) & =\frac{1}{\frac{0.083}{2}+\frac{0.035}{2}} \\
& =\frac{1}{0.0589} \\
& =16.97
\end{aligned}
$$

\subsection{Considerações Finais}

Neste capítulo foram apresentadas os aspectos, princípios e algumas medidas objetivas de interessabilidade de regras, já estudados anteriormente por Horst em (Horst, 1999). Outras medidas de interessabilidade, objetivas ou subjetivas, podem ser encontradas em (Hilderman and Hamilton, 1999).

Num processo de análise quantitativa de regras, as medidas objetivas de interessabilidade podem ser usadas como uma espécie de filtro para selecionar regras potencialmente interessantes e, posteriormente, submeter essas regras a uma avaliação subjetiva, determinando assim qual conhecimento é realmente interessante para o usuário.

No intuito de efetuar a análise qualitativa, implementando as medidas de avaliação apresentadas no Capítulo 3, e também efetuar a análise quantitativa, implementando as medidas objetivas de interessabilidade aqui apresentadas, é proposto neste trabalho o 
Módulo de Análise de Regras. Esse módulo faz parte de um sistema protótipo denominado $\mathcal{R}_{\text {ule }} \mathcal{S}_{\text {ystem }}$, que é apresentado no próximo capítulo. 


\section{Capítulo 5}

\section{O Sistema Protótipo $\mathcal{R}_{\text {ule }} \mathcal{S}_{\text {ystem }}$}

\subsection{Considerações Iniciais}

Como mencionado anteriormente, encontra-se em desenvolvimento no Laboratório de Inteligência Computacional — LABIC - um projeto de grande porte denominado DisCOVER, inicialmente proposto por Baranauskas e Batista (Baranauskas and Batista, 2000). O projeto Discover tem como objetivo fornecer um ambiente integrado para apoiar as etapas do processo de descoberta de conhecimento, oferecendo funcionalidades voltadas para Aprendizado de Máquina (Batista, 1997; Caulkins, 2000; Milaré, 2000; Martins, 2001; Pila, 2000), Mineração de Dados (Batista, 2000; Félix, 1998; Horst, 1999; Lee, 2000; Nagai, 2000; Pugliesi, 2001; Baranauskas, 2001) e Mineração de Textos (Imamura, 2001).

As funcionalidades voltadas para AM consideram, entre outros, um formato padrão para as regras induzidas por algoritmos de AM simbólico, denominado $\mathcal{P B} \mathcal{M}$ (Prati et al., 2001b; Prati et al., 2001a) bem como um formato padrão para os exemplos utilizados (Batista, 2001).

No intuito de testar algumas idéias que poderão futuramente ser implementadas no ambiente Discover, foi proposto inicialmente em (Gomes, 2001) e (Bernardini, 2001) um sistema computacional protótipo denominado $\mathcal{R}_{\text {ule }} \mathcal{S}_{\text {ystem }}$, implementado na linguagem de programação lógica Prolog. Uma visão geral desse sistema, do qual faz parte o Módulo de Análise de Regras proposto neste trabalho, é apresentada a seguir. 


\subsection{Visão Geral do $\mathcal{R}_{\text {ule }} \mathcal{S}_{\text {ystem }}$}

O $\mathcal{R}_{\text {ule }} \mathcal{S}_{\text {ystem }}$ está implementado na linguagem de programação lógica Prolog (Bratko, 1990) mais especificamente em LPA-PROLOG (Westwood, 2000b; Westwood, 2000a). A escolha da linguagem Prolog deve-se ao fato de ser uma linguagem apropriada para o desenvolvimento de protótipos (Monard et al., 1997) nos quais novas idéias possam ser facilmente testadas antes de serem implementadas em um sistema de grande porte. Uma outra característica importante de Prolog é a possibilidade de realizar consultas para obter informações que não ficam restritas àquelas pré-definidas no sistema.

As funcionalidades implementadas no $\mathcal{R}_{\text {ule }} \mathcal{S}_{\text {ystem }}$ possibilitam efetuar a análise automática de regras de conhecimento, induzidas por algoritmos de Aprendizado de Máquina simbólico, e ainda efetuar a avaliação de diversas formas de combinar classificadores simbólicos, bem como, explicar a classificação de novos exemplos realizada por um ensemble de classificares simbólicos. Os dois módulos principais do $\mathcal{R}_{\text {ule }} \mathcal{S}_{\text {ystem }}$, que implementam essas funcionalidades, são:

1. Módulo de Análise de Regras (MAR), que tem como principal objetivo implementar medidas de avaliação e de interessabilidade de regras (apresentadas, respectivamente, nos Capítulos 3 e 4) para efetuar a análise qualitativa e quantitativa de regras induzidas por algoritmos de AM simbólico; e

2. Módulo de Combinação e Explicação (MCE), que tem como objetivo de construir, dado um conjunto de classificadores simbólicos, um outro classificador simbólico que contenha regras desse conjunto de classificadores que sejam as "melhores" segundo as medidas de qualidade de regras implementadas no MAR. Outro objetivo deste módulo é fornecer ao usuário, dado um conjunto de classificadores simbólicos e um exemplo do domínio dos classificadores, quais as regras que foram disparadas nesses classificadores e qual a "melhor" regra, segundo, também, alguma das medidas de avaliação de regras implementadas no MAR. Em outras palavras, fornecer ao usuário uma explicação da classificação.

O $\mathcal{R}_{\text {ule }} \mathcal{S}_{\text {ystem }}$ foi desenvolvido de forma conjunta com Flávia Cristina Bernardini e também é apresentado em (Bernardini, 2002). O Módulo de de Análise de Regras, proposto neste trabalho, está descrito no próximo capítulo. O Módulo de Combinação e 
Explicação é detalhado em (Bernardini and Monard, 2002b).

Na próxima seção são descritos os dados de entrada utilizados pelo $\mathcal{R}_{\text {ule }} \mathcal{S}_{\text {ystem }}$.

\subsection{Os Dados de Entrada do $\mathcal{R}_{\text {ule }} \mathcal{S}_{\text {ystem }}$}

Foi visto que as funcionalidades voltadas para AM, oferecidas no projeto Discover consideram, entre outros, um formato padrão de regras induzidas por diferentes algoritmos de AM simbólico e também um formato padrão para os exemplos utilizados. O formato padrão de regras e exemplos do projeto Discover é sucintamente descrito na seção 5.3.1.

O $\mathcal{R}_{\text {ule }} \mathcal{S}_{\text {ystem }}$ é um sistema computacional protótipo, que pode ser utilizado para testar idéias a serem implementadas no projeto DisCover e que requer como dados de entrada:

1. um ou vários conjuntos de regras induzidas por algoritmos de AM simbólico; e

2. um conjunto de exemplos.

Como o $\mathcal{R}_{\text {ule }} \mathcal{S}_{\text {ystem }}$ foi implementado utilizando a linguagem lógica Prolog, os dados de entrada do sistema foram descritos em uma sintaxe elaborada de acordo com as especificações da linguagem Prolog, que é equivalente à sintaxe padrão de regras e exemplos adotada no projeto Discover. Essa sintaxe equivalente, denominada de sintaxe padrão Prolog, foi proposta de forma conjunta com Flávia Cristina Bernardini e Gustavo Batista em (Gomes et al., 2002).

A conversão do formato padrão de regras e exemplos, do projeto Discover para a sintaxe padrão Prolog, é implementada através de scripts Perl. A Figura 5.1 ilustra a interação desses scripts na conversão da sintaxe de diferentes conjuntos de regras e de exemplos, no formato padrão do Discover $(\mathcal{P B M})$, para a sintaxe padrão Prolog.

A sintaxe padrão Prolog de exemplos e regras está sucintamente descrita nas seções 5.3.2 e 5.3.3, respectivamente.

\subsubsection{O Formato Padrão de Exemplos e Regras do Projeto Dis- cover}

A sintaxe padrão de exemplos adotada no projeto Discover, proposta em (Batista, 2001), é uma extensão do formato dos arquivos de nomes e dados do $\mathcal{C} 4.5$ (Quinlan, 1993) 


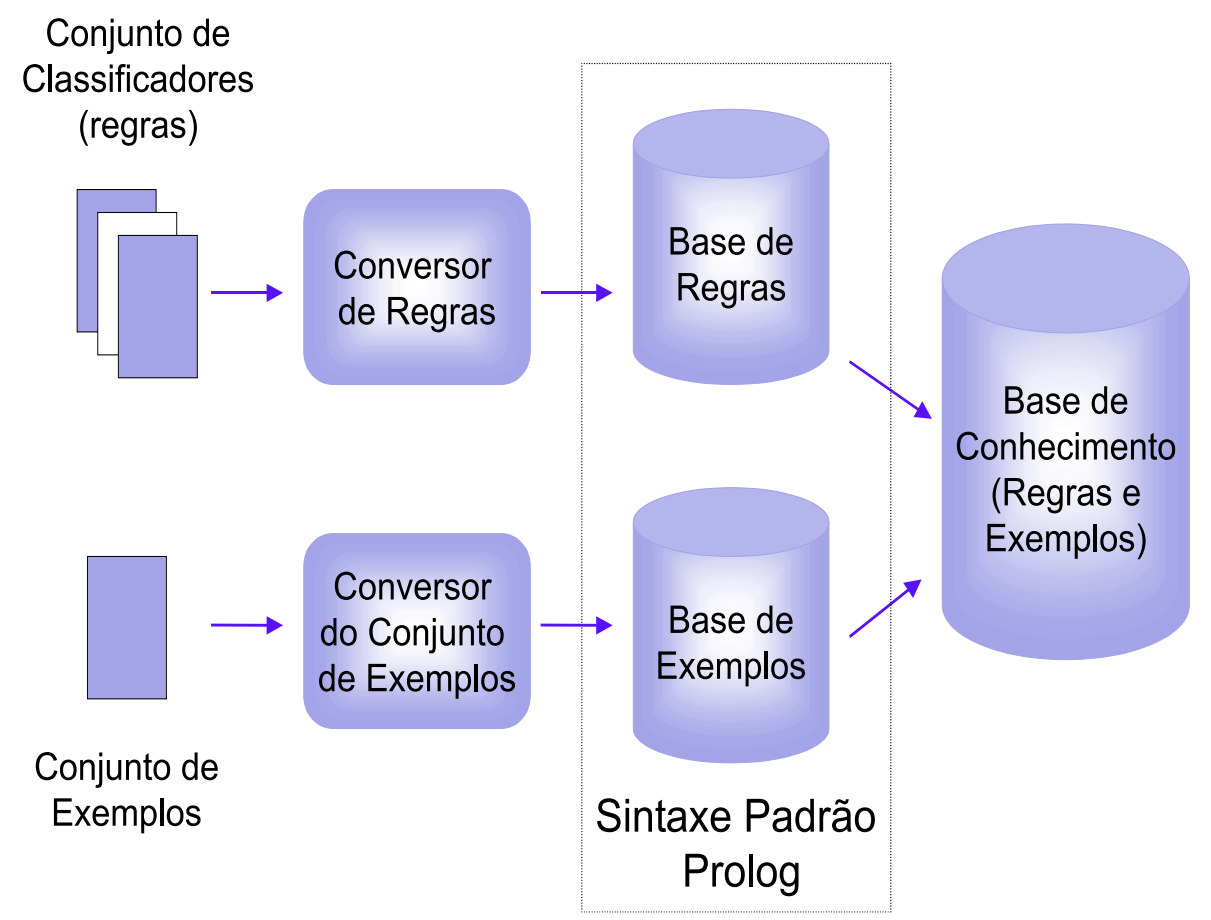

Figura 5.1: Conversores do Formato Padrão $\mathcal{P B M}$ para Sintaxe Padrão Prolog

e utiliza arquivos do tipo texto para declarar os nomes dos atributos (e seus respectivos domínios) bem como os valores que esses atributos assumem no conjunto de exemplos.

Os nomes dos atributos são declarados em um arquivo de nomes, designado com a extensão .names. Os valores que esses atributos assumem no conjunto de exemplos são declarados no arquivo de dados, designados com a extensão .data. Um conjunto de exemplos somente está na sintaxe padrão se estiverem presentes os dois arquivos (.names e .data) que devem possuir o mesmo nome, se diferenciando apenas pela extensão. Para maiores detalhes sobre o formato padrão de exemplos do projeto Discover veja (Batista, 2001; Gomes et al., 2002).

No intuito de unificar a linguagem de representação do conhecimento induzido (classificador) por diferentes algoritmos de AM simbólico, foi proposto em (Prati et al., 2001b) o formato padrão de regras $\mathcal{P} \mathcal{B M}$. Para realizar essa unificação de linguagem de representação foi implementada uma biblioteca composta por uma série de scripts Perl, detalhes descrita em (Prati et al., 2001a; Prati et al., 2001b).

Logo após, derivadas de uma tabela de contingência — Seção 3.2.3, página 22 — foi definido um conjunto mínimo de informações que permitem calcular medidas de avaliação 
de regras. Esse conjunto de informações é obtido através da avaliação de cada uma das regras do conjunto de regras que constituem o classificador, no formato padrão de regras $\mathcal{P B M}$, utilizando um conjunto de exemplos fornecido pelo usuário. Como uma extensão do formato $\mathcal{P} \mathcal{B M}$, após serem calculadas, essas informações são adicionadas ao final de cada uma das regras. O formato padrão proposto para as informações adicionais é o seguinte:

$$
\left[f_{h b}, f_{\bar{h} b}, f_{\overline{h b}}, f_{h \bar{b}}, n\right]
$$

onde $n$ é o número total de exemplos e $f$ representa freqüências relativas dos valores $h b$, $\bar{h} b, \overline{h b}$ e $h \bar{b}$ (tabela de contingência - Seção 3.2.3, página 22).

O formato $\mathcal{P B M}$ estendido contém as freqüências relativas $f_{h b}, f_{\bar{h} b}, f_{\overline{h b}}, f_{h \bar{b}}$, além do número de exemplos, $n$, utilizado no cálculo dessas métricas. Deve ser observado que tanto valores conhecidos como valores desconhecidos nos exemplos são considerados no cálculo dessas métricas. Dessa forma, $h b$, os valores $\bar{h} b, \overline{h b}$ e $h \bar{b}$ podem ser facilmente obtidos multiplicando as freqüências relativas por $n$.

\subsubsection{Sintaxe Padrão Prolog de Exemplos}

A conversão da sintaxe padrão de exemplos do projeto DiSCOver para a sintaxe padrão Prolog é feita através de um script Perl — Figura 5.1 na página anterior implementado por Gustavo Batista (Batista, 2001). Por sua vez, esse script utiliza uma biblioteca que implementa um grupo de sub-rotinas de manipulação de conjuntos de dados na sintaxe padrão. A Figura 5.2 apresenta a gramática que define a sintaxe padrão Prolog para um conjunto de exemplos.

Como visto anteriormente, um conjunto específico de exemplos na sintaxe padrão está definido através das informações contidas nos arquivos . data e . names.

Assim, o predicado ex/2 (Figura 5.3) é utilizado para representar em Prolog cada um dos exemplos contidos no arquivo . data. Os dois argumentos do predicado ex/2 são:

< arg-1 > posição (ou número ) do exemplo no arquivo . data, começando a partir de 0 (zero);

$<$ arg-2 $>$ lista com os valores que cada atributo pode assumir. A ordem desses valores na lista está dada pela ordem em que os atributos estão declarados no arquivo .names. 


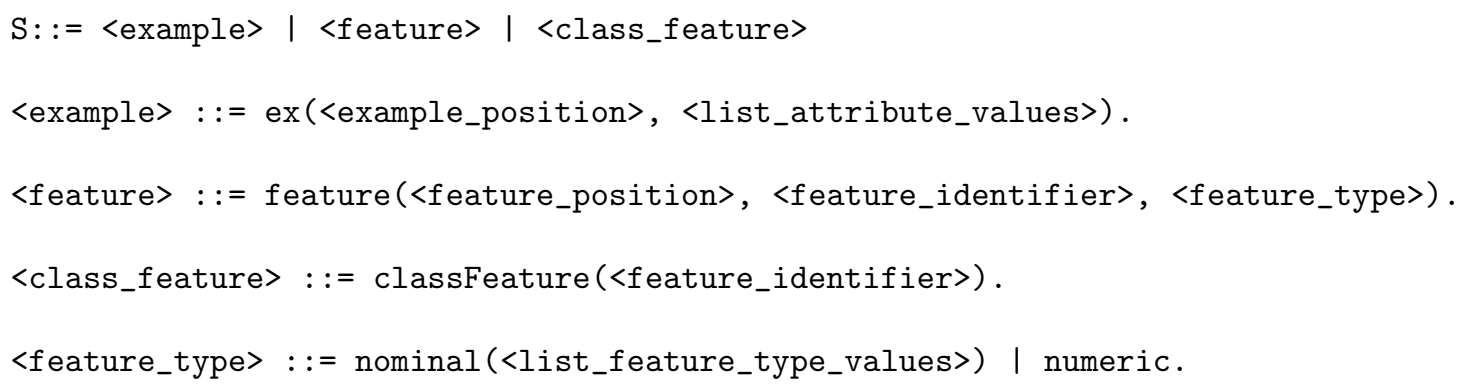

Figura 5.2: Gramática da Sintaxe Padrão Prolog para Exemplos

Os predicados feature/3 e classFeature/1 (Figura 5.3) são utilizados para representar em Prolog as informações sobre os atributos contidos no arquivo . names.

Os três argumentos do predicado feature/3 são:

$<$ arg-1 > posição desse atributo no conjunto de dados. A ordem dos atributos é a mesma que a declarada no arquivo . names, começando em zero.

$<$ arg-2 $>$ nome do atributo. O conversor da sintaxe padrão para a sintaxe padrão Prolog é responsável por verificar se o nome do atributo é um identificador Prolog válido. Por exemplo, no caso do nome do identificador iniciar com uma letra maiúscula (o que tornaria o nome do atributo em uma variável Prolog), o conversor é responsável por substituir por uma letra minúscula.

<arg-3> tipo do atributo. Existem dois tipos básicos na sintaxe padrão Prolog: numeric e nominal. O tipo de dados nominal é declarado na forma de um functor Prolog. Os argumentos do functor são os valores que um atributo nominal pode assumir. $\mathrm{O}$ conversor da sintaxe padrão de exemplos para a sintaxe padrão Prolog é responsável por verificar quais valores um atributo nominal pode assumir. Maiores detalhes sobre os tipos de atributos considerados no projeto Discover podem ser vistos em (Batista, 2001) e ainda, sobre a conversão desses tipos para os tipos de atributos da sintaxe padrão Prolog, podem ser vistos em (Gomes et al., 2002).

O argumento do predicado classFeature/1 é simplesmente o nome da feature que corresponde à classe. A Figura 5.3 mostra o conjunto de exemplos de teste Voyage apre- 
sentados na Tabela 3.5 - Seção 3.3.1, página 26 - representado na sintaxe padrão Prolog.

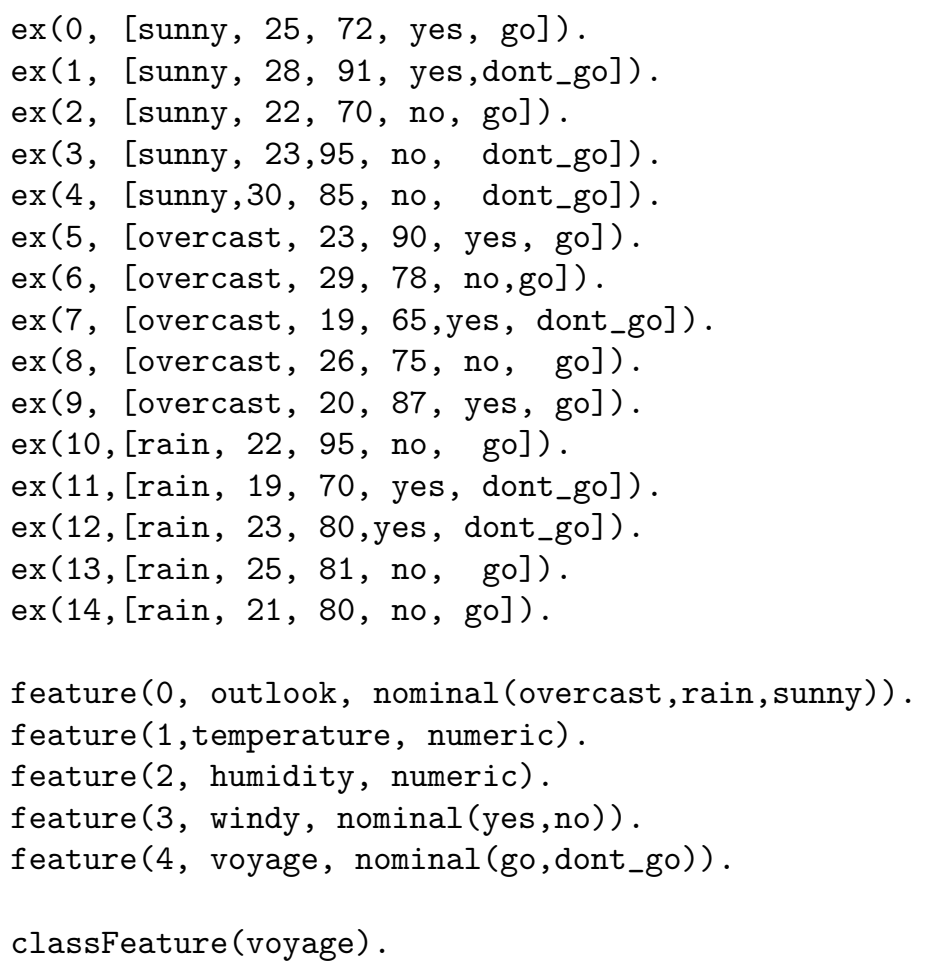

Figura 5.3: Representação Prolog do Conjunto de Exemplos de Teste Voyage

\subsubsection{Sintaxe Padrão Prolog de Regras}

A sintaxe padrão Prolog de regras foi proposta de forma conjunta com Flávia Critina Bernardini em (Gomes et al., 2002) é definida pela gramática mostrada na Figura 5.4.

A conversão da sintaxe padrão de regras do projeto Discover para a sintaxe padrão Prolog é feita através de um script Perl, denominado Conversor de Regras - Figura 5.1 na página 60 - que tem como entrada um arquivo contendo regras de classificação no formato $\mathcal{P B M}$ ou $\mathcal{P} B \mathcal{M}$ estendido. A Figura 5.5 mostra o conjunto de regras da Tabela $3.4-$ Seção 3.3.1, página 25 - no formato $\mathcal{P} \mathcal{B} \mathcal{M}$ estendido as quais foram avaliadas utilizando o conjunto de exemplos de teste da Tabela 3.5 - Seção 3.3.1, página 26. Deve ser ressaltado que a sintaxe padrão Prolog não contempla as regras induzidas por $\mathcal{O C} 1$ (Murthy et al., 


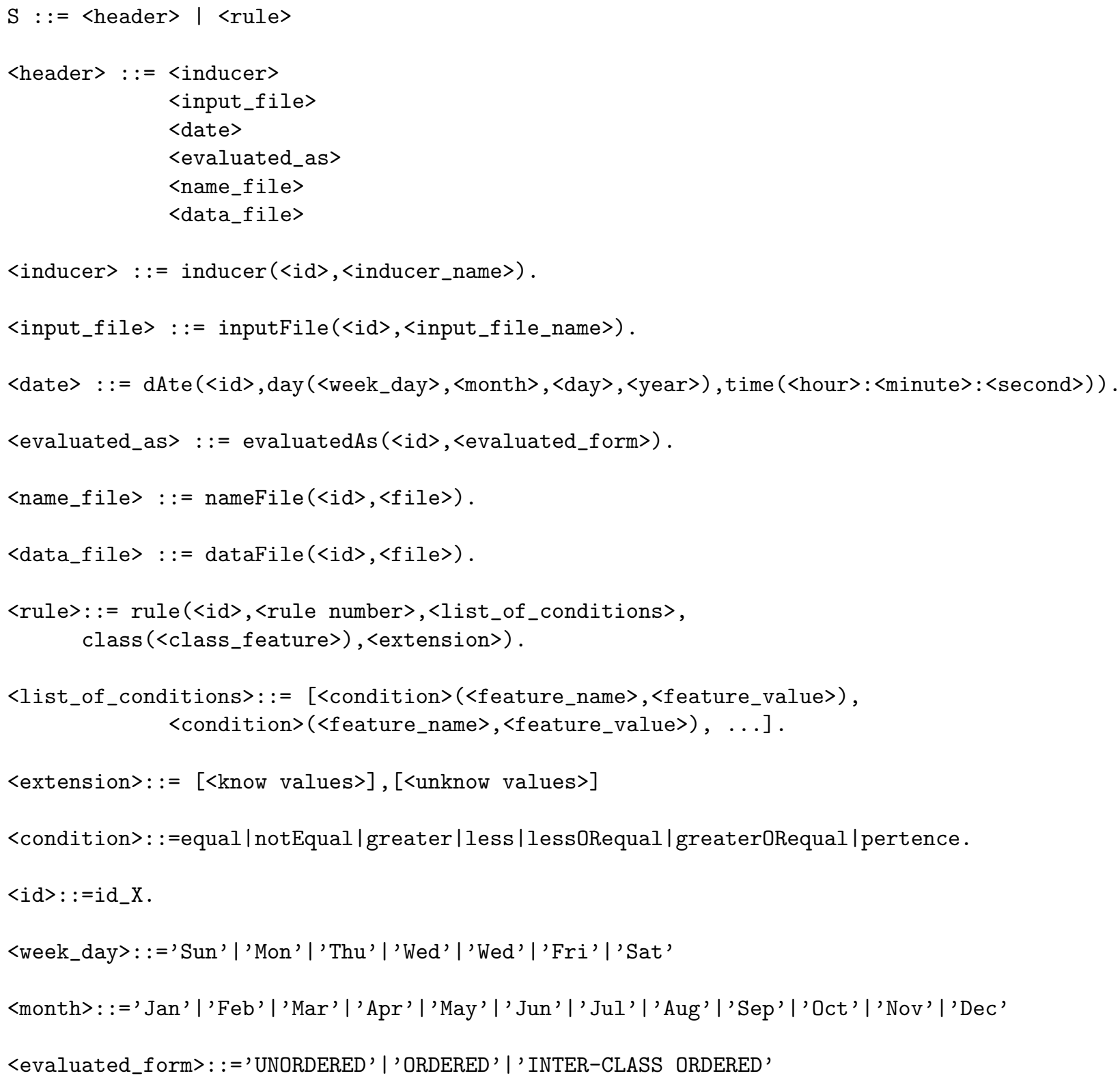

Figura 5.4: Gramática da Sintaxe Padrão Prolog para Regras 
1994). Maiores esclarecimentos sobre essa restrição podem ser obtidos em (Gomes et al., 2002).

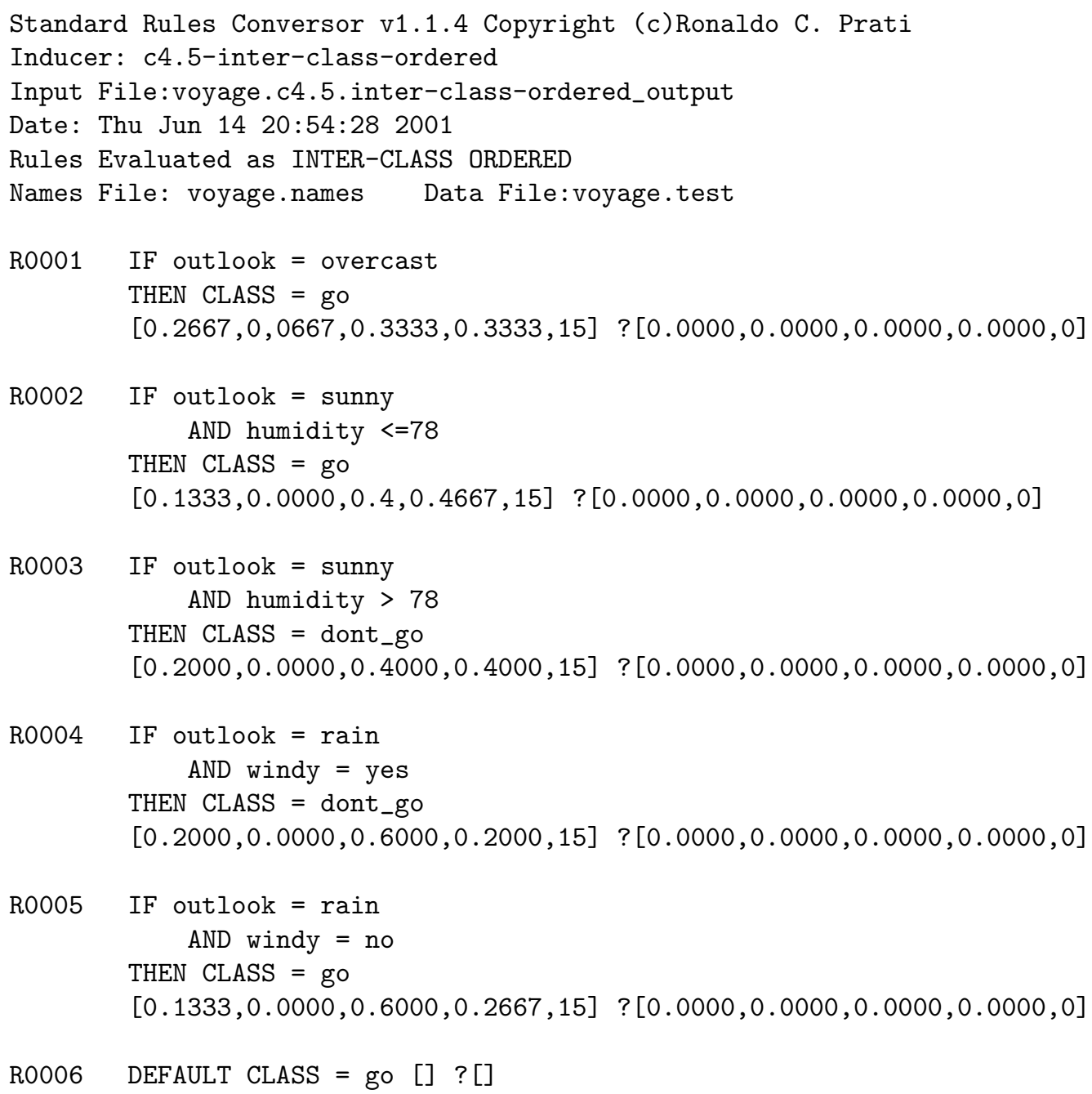

Figura 5.5: Exemplo de um Arquivo de Regras no Formato $\mathcal{P B M}$ Estendido

O conjunto de regras no formato $\mathcal{P B M}$ estendido, ilustrado na Figura 5.5, é apresentado na sintaxe padrão Prolog, após a execução do script Conversor de Regras, na Figura 5.6.

Como mencionado anteriormente, diversos conjuntos de regras podem ser lidos simultaneamente. Assim, é necessário identificar cada um desses conjuntos que provêm de diferentes experiências. Isso é realizado de forma automática pelo Conversor de Regras através 


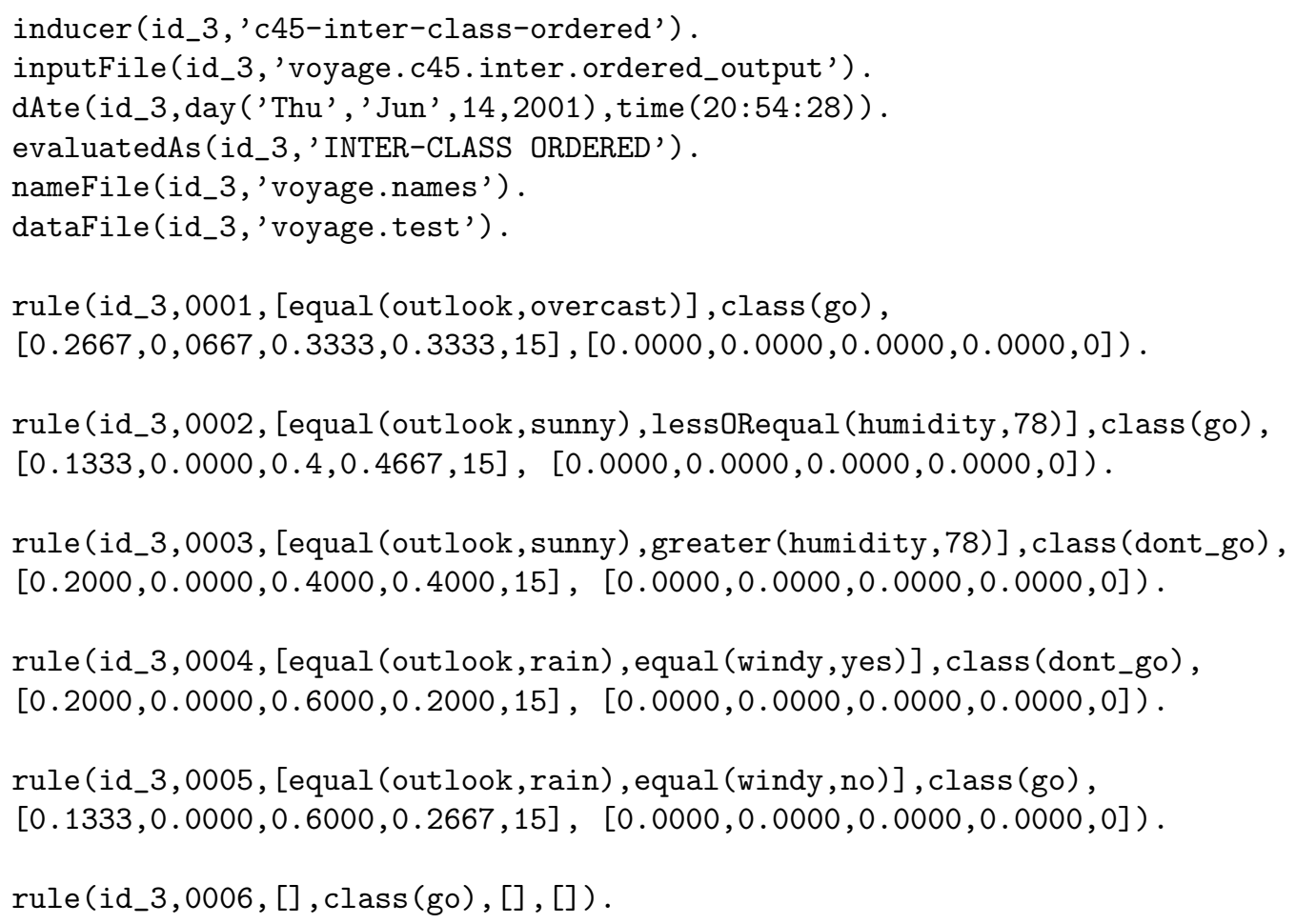

Figura 5.6: Exemplo de um Arquivo de Regras na Sintaxe Padrão Prolog 
do identificador $\langle i d>:$ :=id_X utilizado como primeiro argumento nas cláusulas unitárias Prolog inducer/2, inputFile/2, dAte/3, evaluatedAs/3, namesFile/2, dataFile/2 e rule/6 na Figura 5.4. O valor de X é gerado como o valor da seguinte Função Hash

$$
\mathrm{X}=(\operatorname{Sum} 1+\operatorname{Sum} 2) \bmod 211
$$

onde

$$
\text { Sum } 1=\langle\text { month }>+<\text { day }>+<\text { year }>.
$$

$\mathrm{e}$

$$
\text { Sum } 2=\langle\text { hour }>+<\text { minute }>+<\text { second }>\text {. }
$$

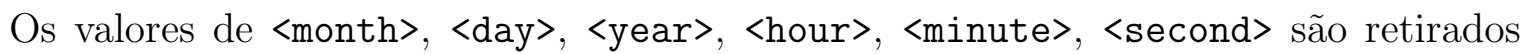
da terceira linha do arquivo no formato $\mathcal{P B M}$ estendido que formam as informações que identificam quando foi realizada essa experiência.

Os símbolos terminais correspondentes a <week_day $>$ e <month> são primeiramente mapeados em números inteiros da seguinte forma:

\begin{tabular}{|cc|cc|}
\hline \hline <week_day> & valor & <month> & valor \\
'Sun' & 1 & 'Jan' & 1 \\
'Mon' & 2 & 'Feb' & 2 \\
'Thu' & 3 & 'Mar' & 3 \\
'Wed' & 4 & 'Apr' & 4 \\
'Wen' & 5 & 'May' & 5 \\
'Fri' & 6 & 'Jun' & 6 \\
'Sat' & 7 & 'Jul' & 7 \\
& & 'Aug' & 8 \\
& & 'Sep' & 9 \\
& & 'Oct' & 10 \\
& & 'Nov' & 11 \\
& & 'Dec' & 12 \\
\hline \hline
\end{tabular}

para finalmente calcular o valor de X, Equação 5.3.3.

Como pode ser observado na Figura 5.6, o predicado inducer/2, informa o indutor utilizado nessa experiência para induzir as regras enquanto que inputFile/2 informa o arquivo que contém as regras no formato $\mathcal{P} \mathcal{B} \mathcal{M}$.

O predicado dAte/3 informa quando as experiências foram realizadas e o predicado evaluatedAs/2 informa como devem ser avaliadas as regras (Baranauskas and Monard, 
2000).

Os predicados nameFile/2 e dataFile/2 informam os os arquivos .names e .data utilizados.

O predicado rule/6 que representa as regras na sintaxe padrão Prolog, possui os seguintes argumentos:

$<$ arg-2 $>$ número da regra.

< arg-3 > lista de condições da regra. No formato padrão $\mathcal{P} \mathcal{B M}$, o <complex> de uma regra é uma disjunção de conjunções de testes de atributos na forma:

<atributo><operador ><valor >

o qual é convertido, na sintaxe padrão Prolog, para:

$<$ condition $>$ (<feature_name $>$, <feature_value $>$ ).

onde <atributo> é mapeado para <feature_name>, <operador> é mapeado, conforme a Tabela 5.1, para <condition> e <valor > é mapeado para <feature_value>. $<$ arg-4 $>$ classe da regra.

$<$ arg-5 $>$ lista de infomações adicionais calculadas sobre exemplos que possuem valores conhecidos.

$<$ arg-6 $>$ lista de infomações adicionais calculadas sobre exemplos que possuem valores desconhecidos.

Para a regra DEFAULT, os argumentos $<$ arg-3 $>,<$ arg-5 $>$ e $<$ arg-6 $>$ são sempre listas vazias.

Conforme mencionado anteriormente, além do formato $\mathcal{P} \mathcal{B M}$ estendido, o Conversor de Regras também suporta regras no formato $\mathcal{P B M}$ (Prati et al., 2001b). Deve ser observado que $<$ arg-5 $>$ e $<$ arg-6 $>$ são sempre listas vazias na conversão de regras no formato padrão $\mathcal{P B} \mathcal{M}$ para a sintaxe padrão Prolog.

Como mencionado anteriormente, vários conjunto de regras e apenas um conjunto de exemplos, ambos na sintaxe padrão Prolog, podem ser carregados na Base de Fatos do $\mathcal{R}_{\text {ule }} \mathcal{S}_{\text {ystem. }}$. A seguir é apresentada a arquitetura desse sistema. 


\begin{tabular}{c|c}
\hline \hline operador & condition \\
\hline$=$ & equal \\
$>=$ & greaterORequal \\
$<=$ & lessORequal \\
$>$ & greater \\
$<$ & less \\
in & pertence \\
$<>$ & notEqual \\
\hline \hline
\end{tabular}

Tabela 5.1: Valores Nominais de Operadores

\subsection{Arquitetura do $\mathcal{R}_{\text {ule }} \mathcal{S}_{\text {ystem }}$}

A Figura 5.7 mostra a arquitetura do $\mathcal{R}_{\text {ule }} \mathcal{S}_{\text {ystem. }}$. Os dois módulos principais (MAR e MCE) consistem de um conjunto de procedimentos Prolog específicos de cada módulo. O Módulo Auxiliar (MA) contém procedimentos para ler arquivos especificados pelo usuário - arquivos que contém conjuntos de regras e o conjunto de exemplos na sintaxe padrão Prolog — bem como uma biblioteca de procedimentos Prolog auxiliares, os quais são compartilhados pelos procedimentos pertencentes ao MAR e ao MCE. Deve ser observado que o usuário não tem acesso aos procedimentos auxiliares do MA.

A Base de Fatos (BF) é utilizada para armazenar os dados de entrada (um ou mais conjuntos de regras e um único conjunto de exemplos) bem como para armazenar informações adicionais, resultantes da execução de algum procedimento ativado pelo usuário.

É importante observar que o $\mathcal{R}_{\text {ule }} \mathcal{S}_{\text {ystem }}$ é um sistema interativo, guiado pelas necessidades do usuário, ou seja, o usuário pode ativar os procedimentos principais de cada um dos três módulos.

A Tabela 5.2 mostra os procedimentos principais (<nome do procedimento $>/<$ aridade>), acessíveis ao usuário, implementados em cada módulo do $\mathcal{R}_{\text {ule }} \mathcal{S}_{\text {ystem }}$.

\begin{tabular}{c|c|c}
\hline MA & MAR & MCE \\
\hline \hline loadRules/0 & evaluateAllSetOfRulesFrequency/1 & rco/4 \\
\hline loadExamples/0 & indAttSurp/1 & classifyExample/2 \\
\hline verifyBase/0 & calculateMeasuresOverAllSetOfRules/0 & confusionMatrix/2 \\
\hline & smallDisjSurpr/3 & classifyExampleByEnsemble/2 \\
\hline & & $\mathrm{kFoldCrossValidation/4}$ \\
\hline
\end{tabular}

Tabela 5.2: Procedimentos Principais do $\mathcal{R}_{\text {ule }} \mathcal{S}_{\text {ystem }}$

Para a maioria desses procedimentos, há um conjunto de pré-condições que devem ser 


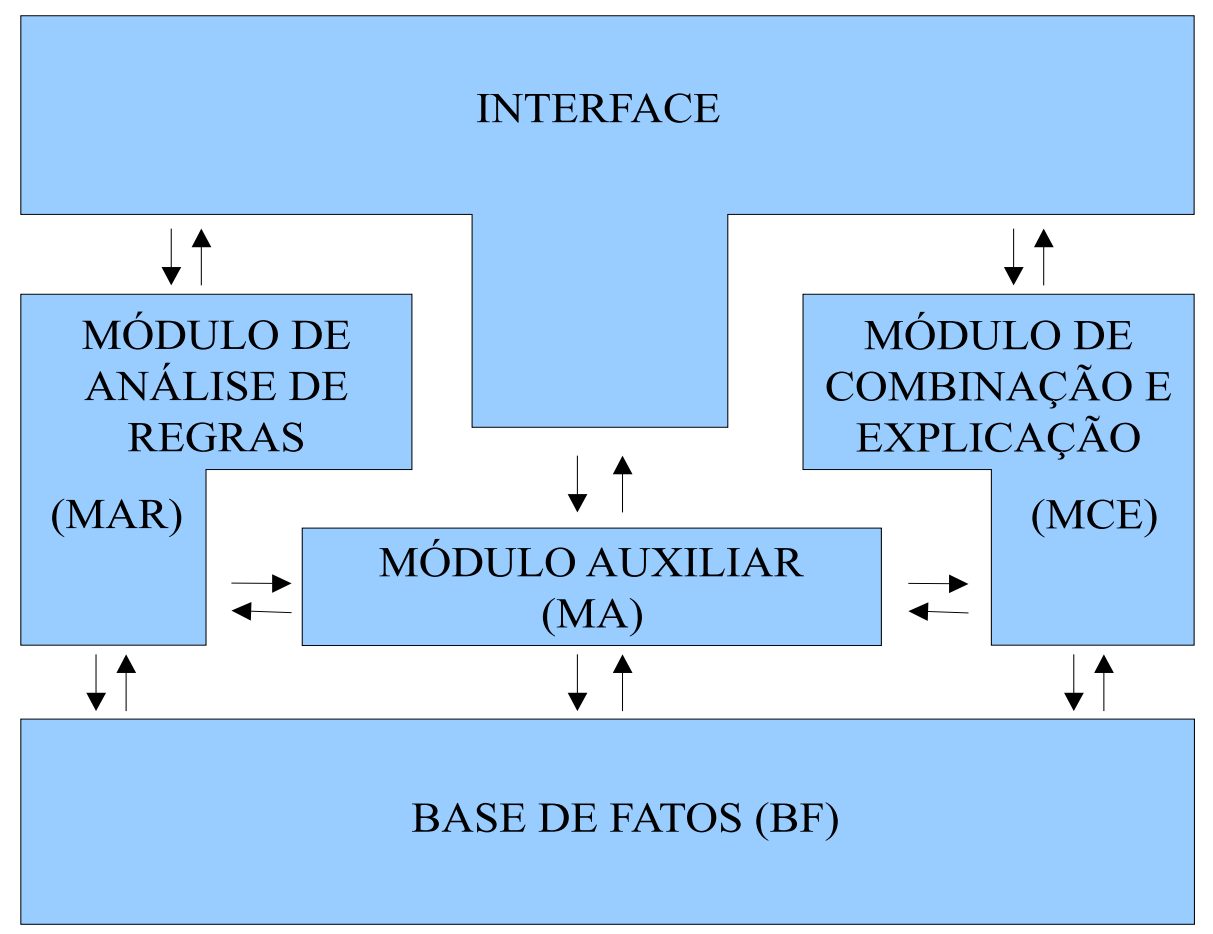

Figura 5.7: Arquitetura do $\mathcal{R}_{\text {ule }} \mathcal{S}_{\text {ystem }}$

satisfeitas para sua correta execução. Também, vários desses procedimentos apresentam um conjunto de pós-condições. Essas condições (tanto pré-condições para disparar um procedimento quanto pós-condições geradas após a execução de um procedimento) são armazenadas na Base de Fatos.

Com o objetivo de documentar o $\mathcal{R}_{\text {ule }} \mathcal{S}_{\text {ystem }}$, foi desenvolvida uma metodologia específica, descrita a seguir.

\subsection{Metodologia para Documentação do $\mathcal{R}_{\text {ule }} \mathcal{S}_{\text {ystem }}$}

Quando foi iniciada a implementação do $\mathcal{R}_{\text {ule }} \mathcal{S}_{\text {ystem }}$ foram pesquisadas na literatura algumas metodologias que pudessem servir para documentar o sistema. Entretanto, nenhuma das metodologias pesquisadas foi suficientemente adequada para atender as especificidades de representação de pré e pós-condições de procedimentos em fluxo de execução. Assim, foi decidido, conjuntamente com Flávia Cristina Bernardini (Bernardini and Monard, 2002b) propor uma metodologia de representação, para documentar o $\mathcal{R}_{\text {ule }} \mathcal{S}_{\text {ystem }}$, 
na forma de um Diagrama de Fluxo de Execução de Procedimentos (PEFD — Procedures Execution Flow Diagram). O PEFD do $\mathcal{R}_{\text {ule }} \mathcal{S}_{\text {ystem }}$ é apresentado na Figura 5.8.

\subsubsection{Componentes do Diagrama de Fluxo de Execução de Pro- cedimentos}

Os componentes do PEFD são 3:

1. Procedimentos;

2. Condições; e

3. Fluxos de Execução.

Cada um desses componentes é descrito a seguir.

\section{Procedimentos}

Um procedimento é representado por um retângulo identificado com o nome do procedimento. O módulo ao qual pertence esse procedimento encontra-se no canto superior direito, dentro de um retângulo menor. No caso do $\mathcal{R}_{\text {ule }} \mathcal{S}_{\text {ystem }}$, são 3 os módulos - MA, MAR e MCE. A Figura 5.9 mostra a representação do procedimento evaluateAllSetOfRulesFrequency/1 do Módulo de Análise de Regras. Quando não for permitido ao usuário disparar um procedimento, o retângulo que se refere a esse procedimento encontrar-se preenchido com um tom de cinza claro. Por exemplo, na Figura 5.8, os procedimentos listOfExamples/1 e dictionary/2 não são acessíveis ao usuário.

\section{Condições}

As pré-condições necessárias para a execução de um procedimento, armazenadas previamente na Base de Fatos, bem como as pós-condições de um procedimento, armazenadas na BF após a sua execução, são representadas por círculos identificados por letras, letras seguidas de números, ou números, onde:

- <letra> nomeia um conjunto de condições; 


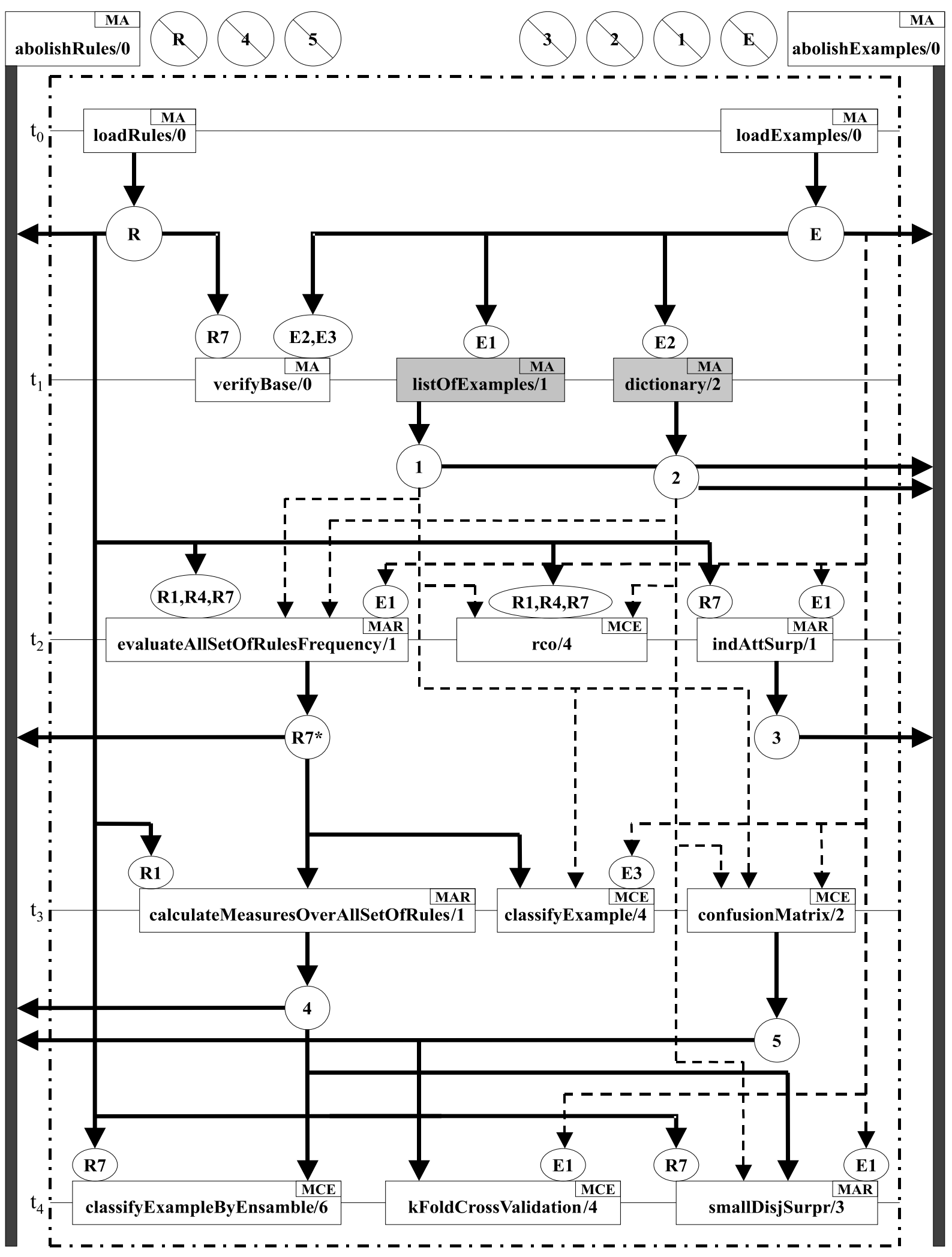

Figura 5.8: Diagrama de Fluxo de Execução de Procedimentos do $\mathcal{R}_{\text {ule }} \mathcal{S}_{\text {ystem }}$ 


\section{evaluateAllSetOfRulesFrequency/1}

Figura 5.9: Exemplo de Representação de um Procedimento

- <letra $><$ númeroc $>$ nomeia a condição <númeroc> que pertence ao conjunto de condições nomeado <letra>; e

- <número> nomeia uma única condição que não pertence a nenhum conjunto de condições.

No $\mathcal{R}_{\text {ule }} \mathcal{S}_{\text {ystem }}$, essas condições são:

$<$ letra $>:=E \mid R$ onde $E=\{E 1, E 2, E 3\}$ e $R=\{R 1, R 2, R 3, R 4, R 5, R 6, R 7\}$.

$<$ número $>:=1|2| 3|4| 5$

O conjunto de condições E e R referem-se, respectivamente, a condições (informações) relacionadas ao conjunto de exemplos e ao conjunto de regras, todas elas na sintaxe padrão Prolog (Gomes and Monard, 2002a). A Figura 5.10 ilustra a representação do conjunto de condições E.

\section{E}

Figura 5.10: Exemplo de Representação de um Conjunto de Condições

Uma descrição dos fatos relacionados a cada condição que pertence ao conjunto de condições E é encontrada na Tabela 5.3.

\begin{tabular}{ll}
\hline E1: ex/2 & $\begin{array}{l}\text { Representa cada um dos exemplos } \\
\text { do conjunto de dados. }\end{array}$ \\
E2: feature/3 & $\begin{array}{l}\text { Representa informaçôes de cada um } \\
\text { dos atributos do conjunto de dados. }\end{array}$ \\
E3: classFeature/1 & $\begin{array}{l}\text { Identifica qual o atributo classe } \\
\text { do conjunto de dados. }\end{array}$ \\
\hline
\end{tabular}

Tabela 5.3: Condições Pertencentes ao Conjunto de Condições E

Analogamente, uma descrição dos fatos relacionados a cada condição que pertence ao conjunto de condições R é encontrada na Tabela 5.4. 


\begin{tabular}{|c|c|c|}
\hline R1: & inducer $/ 2$ & $\begin{array}{l}\text { Identifica o algoritmo de AM utilizado para induzir } \\
\text { as regras. }\end{array}$ \\
\hline R2: & inputFile/2 & $\begin{array}{l}\text { Identifica o arquivo onde encontram-se armazenadas as } \\
\text { regras originais induzidas pelo algoritmo de AM identi- } \\
\text { ficado por R1. }\end{array}$ \\
\hline R3: & dAte $/ 2$ & Identifica data e hora em que as regras foram induzidas. \\
\hline R4: & evaluatedAs/2 & $\begin{array}{l}\text { Indica a forma de avaliação de regras utilizada pelo al- } \\
\text { goritmo de AM identificado por R1. }\end{array}$ \\
\hline R5: & nameFile/2 & $\begin{array}{l}\text { Identifica o arquivo . names relacionado ao conjunto de } \\
\text { exemplos utilizado para induzir as regras. }\end{array}$ \\
\hline R6: & dataFile/2 & $\begin{array}{l}\text { Identifica o arquivo . data relacionado ao conjunto de } \\
\text { exemplos utilizado para induzir as regras. }\end{array}$ \\
\hline R7 : & rule/6 & $\begin{array}{l}\text { Representa cada uma das regras induzidas pelo algorit- } \\
\text { mo de AM utilizado. }\end{array}$ \\
\hline
\end{tabular}

Tabela 5.4: Condições Pertencentes ao Conjunto de Condições R

Quando é necessário representar um subconjunto de um conjunto de condições, esse subconjunto é identificado pelas várias condições que o compõe, separadas por vírgula. Um exemplo pode ser visualizado na Figura 5.11 onde é ilustrado o subconjunto $\{R 1, R 4$ e R7\} de R.

\section{$\mathbf{R} 1, \mathbf{R} 4, \mathbf{R} 7$}

Figura 5.11: Exemplo de Representação de um Subconjunto de Condições

E, finalmente, na Tabela 5.5 segue uma descrição das condições 1, 2, 3, 4 e 5.

Foram também definidos os seguintes dois símbolos de representação do estado de uma condição na BF em um dado instante:

* : para indicar uma pré-condição atualizada pelo procedimento. Isto é, a pré-condição aparece também como pós-condição do procedimento, mas com diferentes informações. Por exemplo, a pré-condição R7 do procedimento evaluateAllSet0fRulesFrequency/1, na Figura 5.8, após a execução do procedimento será atualizada para a póscondição $R 7 *$.

\: indica a eliminação ou inexistência da condição na Base de Fatos. Um exemplo é a inexistência ou eliminação do conjunto de condições R que aparece ao lado do proce- 


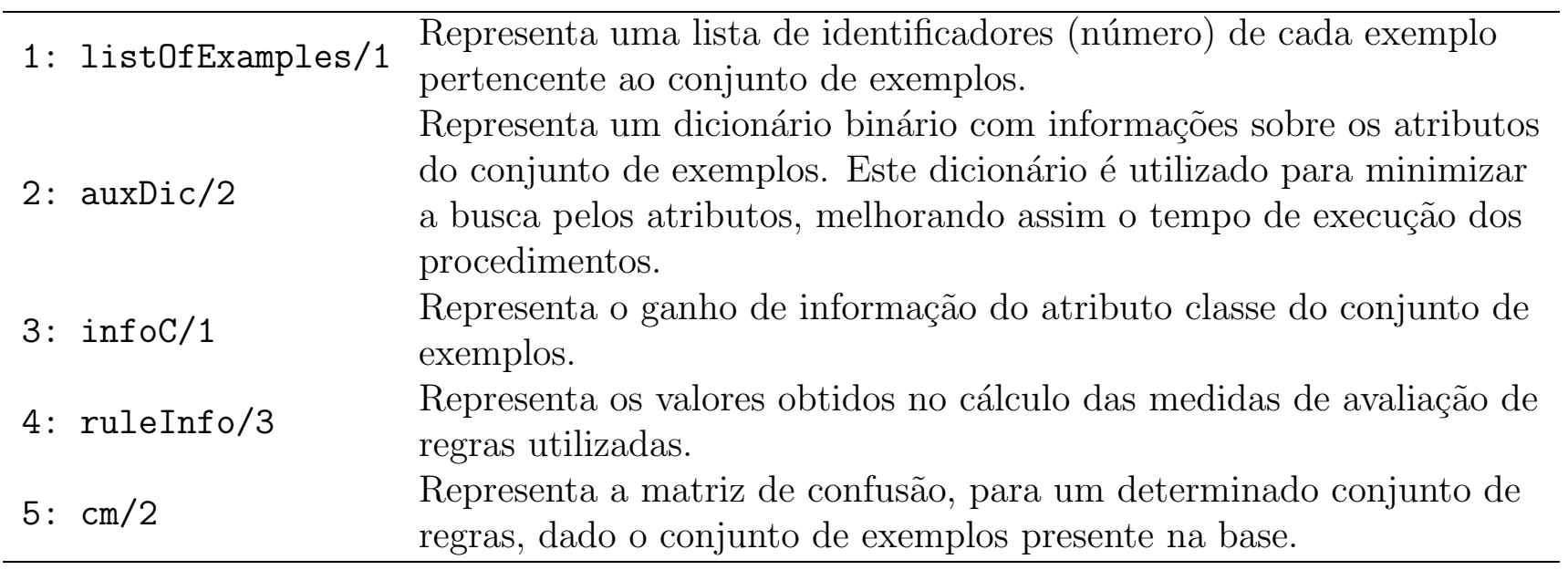

Tabela 5.5: Condições 1, 2, 3, 4 e 5

dimento abolishRules/0 na Figura 5.8. Nesse caso, o conjunto de condições R não existe no estado inicial da base ou foi excluído após a execução de abolishRules/0. O mesmo vale para as condições 4 e 5 .

Além disso, pós-condições de alguns procedimentos podem ser pré-condições para outros procedimentos. Assim, a execução desses procedimentos deve obedecer um fluxo temporal, detalhado a seguir.

\section{Fluxo de Execução}

O fluxo temporal é representado através de linhas de tempo $t_{0}, t_{1}, t_{2}, \ldots, t_{q}$ as quais são somente aplicáveis a procedimentos representados dentro de um retângulo tracejado ${ }^{1}$. Procedimentos fora desse retângulo tracejado são atemporais, ou seja, podem ser executados a qualquer momento.

No caso do $\mathcal{R}_{\text {ule }} \mathcal{S}_{\text {ystem }}$, foram identificadas cinco linhas de tempo, ou seja, $t_{0}, t_{1}, t_{2}, t_{3}$ e $t_{4}$ - Figura 5.8. Nessa figura, pode ser observado que os procedimentos abolishRules/0 e abolishExamples/0 encontram-se fora do retângulo tracejado, portanto eles podem ser executados a qualquer momento. Em outras palavras, são procedimentos atemporais.

Considerando os procedimentos representados dentro do retângulo tracejado, pode-se observar que os procedimentos loadRules/0 e loadExamples/0 na linha $t_{0}$ devem ser executados antes que os procedimentos verifyBase/0, listOfExamples/1 e dictionary/2,

\footnotetext{
${ }^{1}$ tracejado do tipo $\{-\cdot-\cdot-\}$.
} 
na linha $t_{1}$. Analogamente, esses procedimentos da linha $t_{1}$ devem ser executados antes que os procedimentos da linha $t_{2}$, e assim por diante.

Outro aspecto a ser observado é que podem ocorrer relacionamentos entre conjunto de condições e procedimentos, ou entre um conjunto de condições e um subconjunto desse conjunto de condições. Esses relacionamentos são representados por meio de uma linha, contínua ou tracejada ${ }^{2}$, denominada barramento. Existem três tipos de barramento, ilustrados na Figura 5.12, e explicados a seguir:

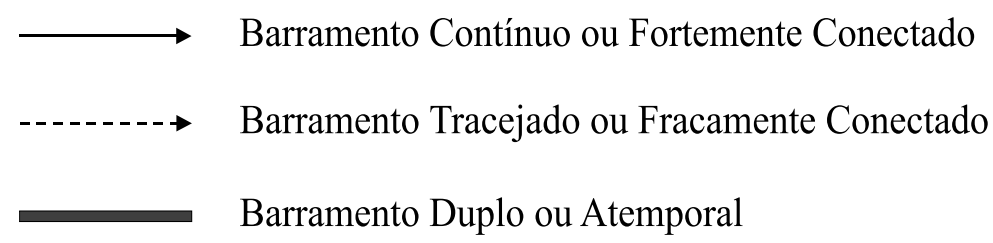

Figura 5.12: Tipos de Barramento

contínuo ou fortemente conectado indica que, se uma pré-condição for apagada da Base de Fatos, todas as pós-condições oriundas de procedimentos relacionados com essa pré-condição devem também ser apagadas da BF.

tracejado ou fracamente conectado indica que, se uma pré-condição for apagada da BF, as pós-condições oriundas de procedimentos relacionados com essa pré-condição não são apagadas da BF.

duplo ou atemporal indica que, ao executar um procedimento relacionado com esse tipo de barramento, todas as condições relacionadas serão apagadas da BF indistintamente.

Quando se relaciona um conjunto de condições a um procedimento, pode-se ter a necessidade de especificar qual é o subconjunto de condições que realmente faz parte do conjunto de pré-condições do procedimento. Para se detalhar esse tipo de informação, substitui-se o relacionamento entre o conjunto de condições e o procedimento em questão por um relacionamento entre o conjunto de condições e o subconjunto de pré-condições.

\footnotetext{
${ }^{2}$ tracejado do tipo $\{---\}$.
} 
Posiciona-se o círculo que representa esse subconjunto de pré-condições sobre o retângulo que representa o procedimento em questão.

Por exemplo, na Figura 5.8, o procedimento verifyBase/0 somente necessita da précondição $R 7$ do conjunto de condições $R$ e do subconjunto $\{E 2$, E3\} de condições do conjunto de condições E.

\subsubsection{Descrição do Fluxo de Execução do $\mathcal{R}_{u l e} \mathcal{S}_{\text {ystem }}$}

Para um melhor entendimento do PEFD do $\mathcal{R}_{\text {ule }} \mathcal{S}_{\text {ystem }}$ (Figura 5.8), aqui é apresentada uma descrição do fluxo de execução dos procedimentos implementados nesse sistema.

Na parte superior da Figura 5.8 está representado o estado inicial da Base de Fatos. O estado inicial é caracterizado pela ausência de todas as condições que podem estar presentes na BF, ou também, pela exclusão de todas essas condições. Dessa forma, os procedimentos abolishRules/0 e abolishExamples/0 são responsáveis por excluir, respectivamente, as condições R, 4 e 5 e E, 1, 2 e 3. A ausência ou a exclusão dessas condições é representada por meio do traço que corta o círculo contendo a identificação de cada condição.

Considerando que nenhuma condição está presente na Base de Fatos, o início do fluxo de execução do $\mathcal{R}_{\text {ule }} \mathcal{S}_{\text {ystem }}$ dá-se no instante $t_{0}$, quando podem ser executados seguidamente, em qualquer ordem, os procedimentos loadRules/0 e loadExamples/0. Por exemplo, na execução de loadRules/0 o conjunto de condições R é gravado na BF. Esse conjunto de condições é fortemente conectado ao barramento atemporal e pode ser removido da BF somente pela execução do procedimento abolishRules/0. Ao ser executado o procedimento loadExamples/0, o conjunto de condições E é gravado na BF. Esse conjunto de condições também é fortemente conectado ao barramento atemporal e apenas a execução do procedimento abolishExamples/0 pode apagar esse conjunto de condições da BF.

Seguindo para o instante $t_{1}$, os procedimentos verifyBase/0, list0fExamples/1 e dictionary/2 podem ser executados seguidamente. O procedimento verifyBase/0 tem como pré-condições subconjuntos de $\mathrm{R}$ e $\mathrm{E}$ aos quais encontram-se fortemente conectados. Especificamente, a pré-condição R7 do conjunto de condições R e o subconjunto de condições E2 e E3 do conjunto de condições E. A conexão forte indica que essas pré- 
condições específicas serão apagadas da BF se o conjunto R e E de condições for apagado da BF.

Ainda no instante $t_{1}$, os procedimentos list0fExamples/1 e dictionary/2 podem ser executados seguidamente em qualquer ordem. Como pode ser observado, os retângulos que identificam esses procedimentos estão preenchidos com um tom de cinza claro, o que significa que não podem ser acessados pelo usuário. Após serem executados, esses procedimentos gravam na BF, respectivamente, as condições 1 ou 2. Essas duas condições estão fortemente conectadas ao barramento atemporal, portanto elas podem ser apagadas somente através da execução do procedimento abolishExamples/0.

Partindo para o instante $t_{2}$, os procedimentos evaluateAllSetOfRulesFrequency/1, rco/4 e indAttSurp/1 podem ser executados em qualquer ordem. Esses procedimentos estão fracamente conectados às pré-condições 1 e 2 e fortemente conectados com os conjuntos de condições R e E.

Por exemplo, o procedimento evaluateAllSet0fRulesFrequency/1 está fracamente conectado às pré-condições 1 e 2, fortemente conectado à condição $R$ através do subconjunto de pré-condições R1, R4, R7 e fracamente conectado com a pré-condição E através da pré-condição E1. Após sua execução, esse procedimento modifica a pré-condição R7 gravando a pós-condição R7* na BF. Isso implica que, se 1, 2 ou E forem apagadas da $\mathrm{BF}$, a pós-condição $\mathrm{R} 7 *$ continua válida. $\mathrm{R} 7 *$ só pode ser apagada pela execução do procedimento abolishRules/0.

O fluxo de execução do PEFD do $\mathcal{R}_{\text {ule }} \mathcal{S}_{\text {ystem }}$ nos instantes $t_{3}$ e $t_{4}$ é semelhante ao descrito no instante $t_{2}$.

Em qualquer dos instantes $t_{0}, \ldots, t_{4}$ os procedimentos atemporais abolishRules $/ 0$ e abolishExamples/0 podem ser executados. Por exemplo, se abolishExamples/0 for executado no instante $t_{2}$, todas as condições presentes na BF até aquele instante e conectadas ao barramento atemporal serão excluídas - no caso, serão excluídas as condições E, 1 e 2. Deve ser observado que os procedimentos atemporais abolishRules/0 e abolishExamples/0 retornam a $\mathrm{BF}$ ao seu estado inicial, ou seja, as condições ligadas a esses procedimentos (respectivamente R, 4 e 5 e E, 1, 2 e 3) são apagadas da BF por meio do barramento fortemente conectado. 


\subsection{Considerações Finais}

Neste capítulo foi apresentado o $\mathcal{R}_{\text {ule }} \mathcal{S}_{\text {ystem }}$, um sistema protótipo inicialmente proposto em (Gomes, 2001) e (Bernardini, 2001), implementado utilizando a linguagem de programação lógica Prolog. Como mencionado anteriormente, para que possam ser utilizadas as funcionalidades implementadas nesse sistema, os dados de entrada do $\mathcal{R}_{\text {ule }} \mathcal{S}_{\text {ystem }}$ devem estar descritos na sintaxe padrão Prolog proposta em (Gomes et al., 2002).

Além da arquitetura do $\mathcal{R}_{\text {ule }} \mathcal{S}_{\text {ystem }}$, também foi apresentada a metodologia PEFD Procedures Execution Flow Diagram, descrita em maiores detalhes em (Gomes and Monard, 2002a; Bernardini and Monard, 2002b), especificamente elaborada para documentar esse sistema. A metodologia PEFD facilita muito o projeto de uma interface gráfica, que será construída futuramente, além de ser adequada à representação de pré e pós condições dos procedimentos implementados em cada módulo do $\mathcal{R}_{\text {ule }} \mathcal{S}_{\text {ystem }}$.

O $\mathcal{R}_{\text {ule }} \mathcal{S}_{\text {ystem }}$ é composto de dois módulos principais e, dentre eles, o Módulo de Análise de Regras (MAR), descrito no próximo capítulo, tem o objetivo de efetuar a análise automática de regras, implementando as medidas de avaliação e de interessabilidade apresentadas, respectivamente, nos Capítulos 3 e 4 deste trabalho. 


\section{Capítulo 6}

\section{O Módulo de Análise de Regras do $\mathcal{R}_{\text {ule }} \mathcal{S}_{\text {ystem }}$}

\subsection{Considerações Iniciais}

O Módulo de Análise de Regras (MAR) é um dos principais módulos do sistema computacional protótipo $\mathcal{R}_{\text {ule }} \mathcal{S}_{\text {ystem }}$, apresentado no capítulo anterior. O MAR tem como objetivo efetuar a análise qualitativa e quantitativa de regras induzidas por diferentes algoritmos de Aprendizado de Máquina simbólico.

A análise qualitativa de regras é efetuada por meio de procedimentos que implementam as medidas de avaliação de regras apresentadas no Capítulo 3. A análise quantitativa de regras é realizada através de procedimentos que implementam as medidas objetivas de interessabilidade de regras apresentadas no Capítulo 4.

A seguir é descrito o Módulo de Análise de Regras do $\mathcal{R}_{\text {ule }} \mathcal{S}_{\text {ystem }}$, bem como os procedimentos que possibilitam a análise de regras sob os aspectos qualitativos e quantitativos.

\subsection{Os Procedimentos Implementados no MAR}

Na Figura 5.8 na página 72, os procedimentos que fazem parte do Módulo de Análise de Regras são identificados por meio da sigla MAR. Esses procedimentos são:

1. Cálculo de Informações de Avaliação de Regras - evaluateAlıSet0fRuleFrequency/1 
2. Cálculo de Medidas de Avaliação de Regras - calculateMeasuresOverAllSet0fRules/1

3. Cálculo do Grau de Surpresa de Pequenos Disjuntos - smallDisjSurp/3

4. Cálculo do Grau de Surpresa dos Atributos Individuais Discretos da Regra - indAttSurp/2

A instanciação ${ }^{1}$ de cada um dos $i$ argumentos de um procedimento de aridade $i$ é descrita utilizando a notação padrão para documentar programas Prolog, ou seja,

$+<$ arg-i $>$ o argumento deve estar instanciado;

- <arg-i > o argumento deve estar desinstanciado, ou seja, é uma variável livre;

? < arg-i $>$ o argumento pode estar instanciado ou desinstanciado.

Os procedimentos implementados no MAR possibilitam a análise de regras sob os aspectos qualitativo e quantitativo. Esses procedimentos são descritos nas próximas seções.

\subsection{Análise Qualitativa de Regras}

Sob o aspecto qualitativo, regras são avaliadas com o objetivo de saber quais são aquelas melhor sustentadas pelos dados (Lavrac et al., 1999). No MAR, a análise qualitativa é efetuada por meio de procedimentos que implementam as medidas de avaliação de regras apresentadas no Capítulo 3.

Conforme mencionado anteriormente na Seção 3.2.1, página 20, neste trabalho, regras tem o formato Body $\rightarrow$ Head ou, resumidamente $B \rightarrow H$, onde $B$ é o corpo da regra e $H$ é a cabeça da regra. Dado um exemplo $\mathbf{x}_{i}$ e a classe $y_{i}$ a qual ele pertence, a avaliação da regra é feita através de um procedimento que determina se $B$ é verdadeiro ou falso e se $H$ é verdadeiro ou falso para esse exemplo. Por meio desse procedimento é

\footnotetext{
${ }^{1} \mathrm{Na}$ realidade, a instanciação dos argumentos dos procedimentos aqui descrita refere-se à instanciação na execução padrão realizada por um usuário do sistema. Entretanto, para o implementador do sistema, a instanciação do tipo + pode, às vezes, ser substituída por uma instanciação do tipo ?, e a do tipo - pode sempre ser substituída por uma instanciação do tipo ?. Esta característica de Prolog permite verificar resultados específicos além de auxiliar o implementador a realizar rapidamente a depuração dos procedimentos.
} 
possível determinar, para cada regra $R_{u}$ de um classificador simbólico $\mathbf{h}=\left\{R_{1}, \ldots, R_{N R}\right\}$, as informações (cardinalidades) $h b, \bar{h} b, \overline{h b}$ e $h \bar{b}$ derivadas da tabela de contingência Seção 3.2.3, página 22 .

No cálculo dessas informações, a forma que as regras do classificador simbólico $\mathbf{h}$ foram induzidas pelo algoritmo de AM deve ser considerada. O Módulo de Análise de Regras calcula essas informações para regras unordered, ordered e interclass, conforme descrito a seguir.

\section{Unordered}

Para um conjunto de regras unordered, essas informações são calculadas verificando a cobertura de cada regra do classificador simbólico $\mathbf{h}=\left\{R_{1}, \ldots, R_{N R}\right\}$ em todo conjunto de exemplos $S$. O Algoritmo 6.1 mostra como é realizado o cálculo dessas informações para um conjunto de regras unordered.

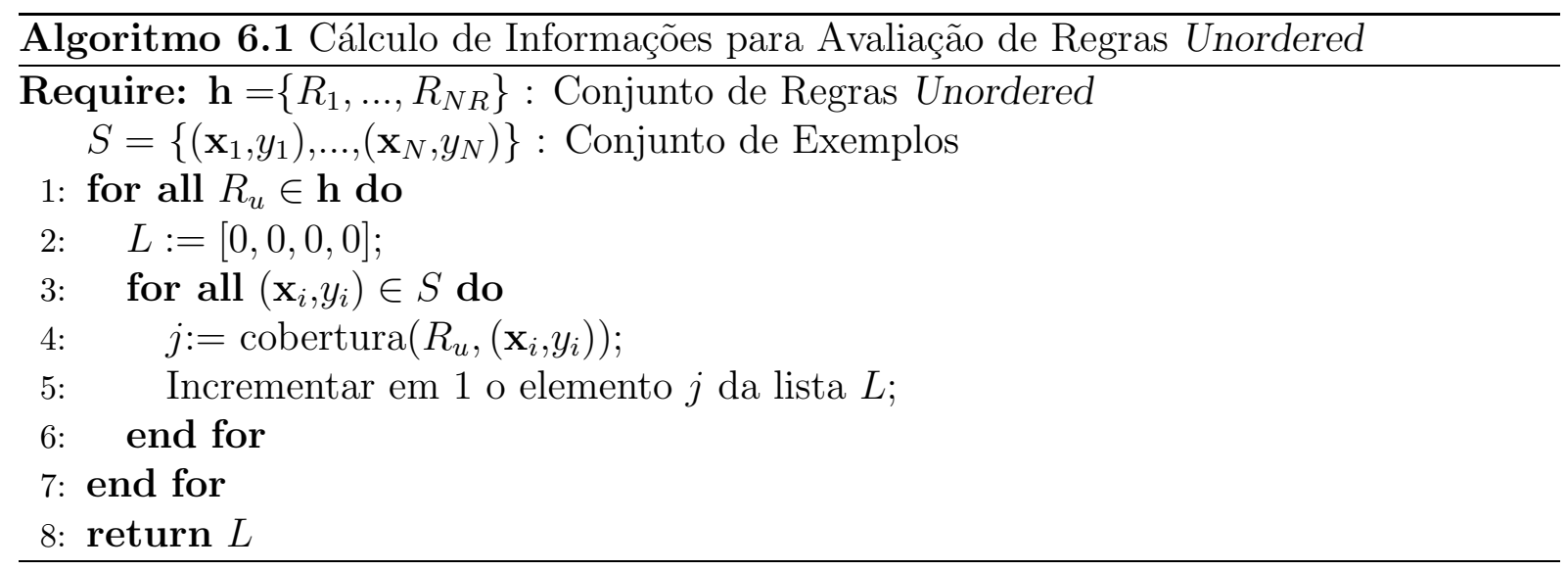

A função cobertura $\left(R_{u},\left(\mathbf{x}_{i}, y_{i}\right)\right)$ retorna um número inteiro no intervalo $1 \ldots 4$ que é usado como índice para o elemento que deve ser atualizado na lista $L$. O índice 1 corresponde a $h b, 2$ a $\bar{h} b, 3$ a $\overline{h b}, 4$ a $h \bar{b}$.

\section{Ordered}

No caso de regras ordered existe um else implícito entre cada regra, assim o exemplo deve ser aplicado a partir da primeira regra, até que uma delas cubra esse exemplo ( $B$ é verdade para o exemplo). Esse exemplo deve ser coberto apenas por essa regra. Para as regras seguintes àquela que cobre o exemplo, as informações devem ser atualizadas incrementando-se os valores de $\bar{b} h$ e $\overline{b h}$ conforme a classe $C_{v}$ do 
exemplo. O Algoritmo 6.2 mostra como é realizado o cálculo dessas informações para um conjunto de regras ordered.

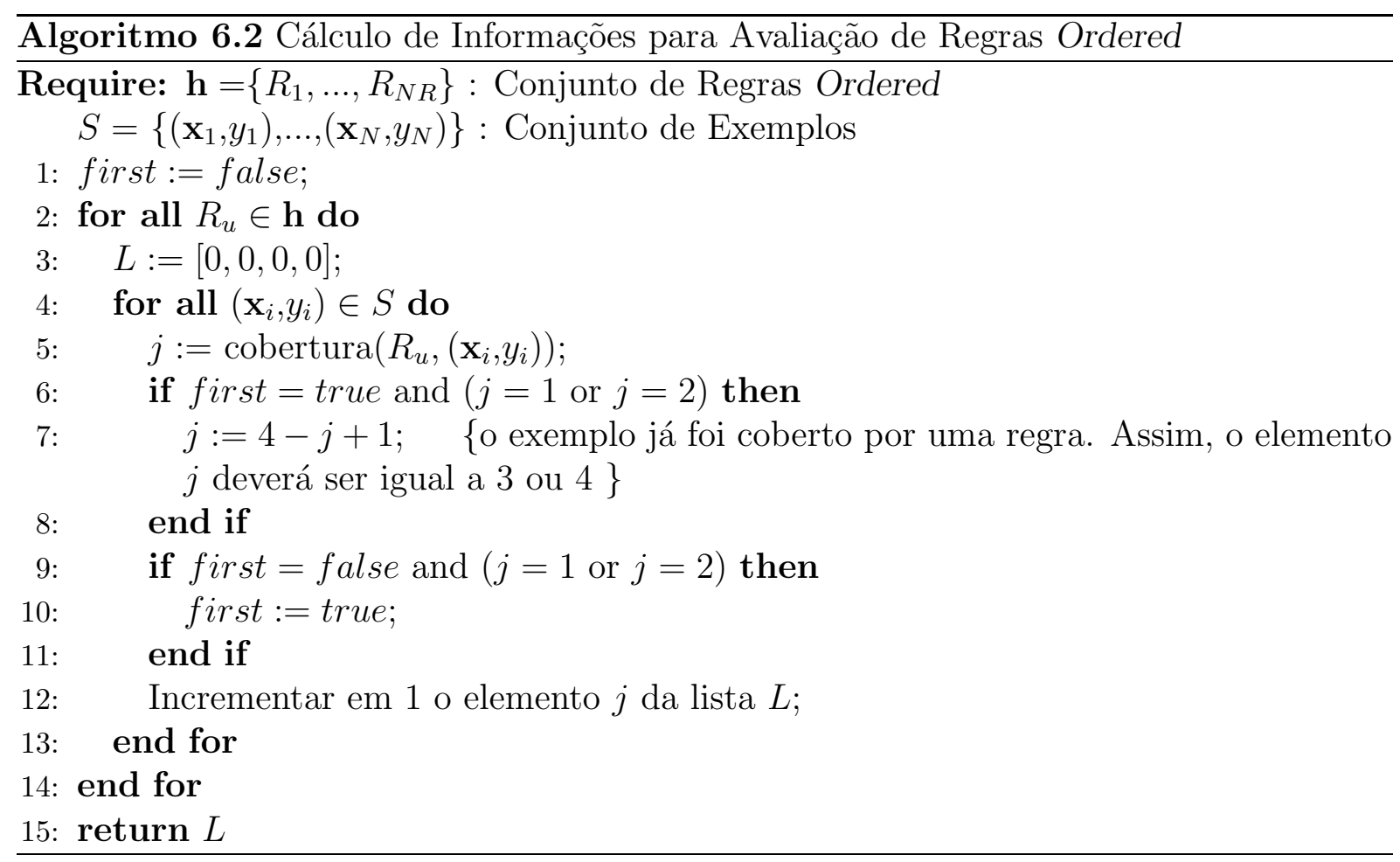

A variável first está associada a cada exemplo $\left(\mathbf{x}_{i}, y_{i}\right)$ e recebe false se o exemplo não foi coberto por uma regra ou true se já foi coberto.

\section{Interclass}

As regras interclass são separadas em $C_{v}$ blocos para cada uma das $C_{v}, v=1, \ldots, N C l$ diferentes classes, existindo um else implícito entre cada um desses blocos. Para regras em um mesmo bloco $C_{v}$, as informações são calculadas como descrito anteriormente para regras unordered. Ao ser encontrada uma regra que cubra o exemplo em um bloco $C_{v}$, as informações extraídas das regras subseqüentes devem ser atualizadas como descrito anteriormente para regras ordered, nos $C_{N C l-v}$ blocos seguintes.

Por exemplo, a Figura 6.1 mostra que, para cada classe, existe um conjunto de subconjuntos de regras $\mathbf{h}^{\prime}=\left\{\mathbf{h}_{1}^{\prime}, \ldots, \mathbf{h}_{N C l}^{\prime}\right\}^{2}$. É importante observar que, além do else implícito entre cada um desses subconjuntos de regras, esses subconjuntos

\footnotetext{
${ }^{2} \mathbf{h}^{\prime}=\mathbf{h}-\{$ default $\}$. A regra default é uma regra especial cujo corpo é vazio e a cabeça é a classe de maior freqüência no conjunto de exemplos. Assim, uma hipótese h sempre irá classificar um exemplo devido à existência da regra default.
} 


$$
\begin{aligned}
& \mathbf{h}_{1}^{\prime} \\
& \left\{\begin{array}{l}
R_{1}: \text { Corpo then CLASS }=\alpha \\
R_{2}: \text { Corpo then CLASS }=\alpha
\end{array}\right. \\
& \text { else } \\
& \mathbf{h}_{2}^{\prime} \\
& \left\{\begin{array}{l}
R_{3}: \text { Corpo then CLASS }=\beta \\
R_{4}: \text { Corpo then CLASS }=\beta \\
R_{5}: \text { Corpo then CLASS }=\beta
\end{array}\right. \\
& \text { else } \\
& \vdots \\
& \left\{\begin{array}{l}
R_{u-2}: \text { Corpo then CLASS }=\gamma \\
R_{u-1}: \text { Corpo then CLASS }=\gamma
\end{array}\right. \\
& \operatorname{com} \alpha, \beta, \gamma \in C=C_{1}, \ldots, C_{N C l} \text {. }
\end{aligned}
$$

Figura 6.1: Regras Interclass — Conjunto de Subconjuntos de Regras

são formados conforme a ordem em que o algoritmo de AM induz as classes que compõem a hipótese $\mathbf{h}$. Cada elemento de $\mathbf{h}^{\prime}$, ou seja, $\mathbf{h}_{1}^{\prime}, \ldots, \mathbf{h}_{N C l}^{\prime}$, é uma hipótese com as regras que predizem a mesma classe.

Assim, o exemplo deve ser aplicado a todas as regras de $\mathbf{h}_{1}^{\prime}$ e as informações são calculadas como descrito anteriormente para regras unordered. Se nenhuma regra de $\mathbf{h}_{1}^{\prime}$ cobrir o exemplo, ele deve ser aplicado a $\mathbf{h}_{2}^{\prime}$ e assim por diante. Ao ser encontrada uma regra em um $\mathbf{h}_{v}^{\prime}$ que cobre o exemplo, as informações extraídas das regras subseqüentes são atualizadas como descrito anteriormente para regras ordered, nos $\mathbf{h}_{\mathrm{NCl-v}}^{\prime}$ subconjuntos seguintes.

Após determinar as informações para cada uma das regras $R_{u}{ }^{3}$ de um classificador simbólico $\mathbf{h}$, os valores encontrados são expressos sob a forma de estimativas de probabilidade (freqüências relativas) e utilizadas para calcular as medidas de avaliação de regras apresentadas no Capítulo 3.

Como já mencionado, os procedimentos do MAR possibilitam a análise qualitativa de regras, implementando o cálculo das informações a serem usadas para determinar

\footnotetext{
${ }^{3} \mathrm{Se} R_{u}$ é a regra default, então as informações não são calculadas para essa $R_{u} \in \mathbf{h}$.
} 
as medidas de avaliação de regras, apresentadas anteriormente no Capítulo 3. Esses procedimentos são:

1. Cálculo de Informações de Regras - evaluateAllSet0fRuleFrequency/1.

2. Cálculo de Medidas de Avaliação de Regras - calculateMeasuresOverAllSetOFRules/1.

O procedimento evaluateAllSet0fRuleFrequency/1 implementa o cálculo de informações para as três formas de indução de regras, conforme mencionado anteriormente. Na Figura 5.8, página 72 , esse procedimento está representado na linha $t_{2}$. O argumento desse procedimento é:

$+<$ arg-1 $>$ yes para avaliar todos os conjuntos de regras, desconsiderando se foram anteriormente avaliados;

no para avaliar somente os conjuntos de regras ainda não avaliados.

Dado um conjunto de classificadores simbólicos $\mathcal{H}=\left\{\mathbf{h}_{1}, \ldots, \mathbf{h}_{L}\right\}$ e um conjunto de exemplos $S=\left\{\left(\mathbf{x}_{1}, y_{1}\right), \ldots,\left(\mathbf{x}_{N}, y_{N}\right)\right\}$, o procedimento evaluateAllSet0fRuleFrequency/1 implementa o cálculo das informações para cada classificador simbólico $\mathbf{h} \in \mathcal{H}$, aplicando todos os exemplos de $S$ em cada regra $R_{u}$ de $\mathbf{h}$. As informações $h b, \bar{h} b, \overline{h b}$ e $h \bar{b}$ são calculadas levando em conta, separadamente, exemplos com valores conhecidos e exemplos com valores desconhecidos. Essas informações são expressas sob a forma de freqüências relativas, i.e. $f_{h b}, f_{\bar{h} b}, f_{\overline{h b}}, f_{h \bar{b}}$, derivadas da tabela de contingência - Seção 3.2.3, página 22.

O procedimento evaluateAllSetOfRuleFrequency/1 então adiciona as informações expressas como freqüências relativas ao final de cada regra $R_{u}$ de cada hipótese $\mathbf{h} \in \mathcal{H}$ e grava cada regra atualizada na Base de Fatos.

No Algoritmo 6.3 é descrito o cálculo das informações implementado no procedimento evaluateAllSetOfRuleFrequency/1.

Dado um conjunto de classificadores simbólicos $\mathcal{H}=\left\{\mathbf{h}_{1}, \ldots, \mathbf{h}_{L}\right\}$ e um conjunto de exemplos $S=\left\{\left(\mathbf{x}_{1}, y_{1}\right), \ldots,\left(\mathbf{x}_{N}, y_{N}\right)\right\}$, o procedimento calculateMeasuresOverAllSetOFRules/1 implementa o cálculo das medidas de avaliação de regra, utilizando as informações calculadas pelo procedimento evaluateAllSetOfRuleFrequency/1.

Esse procedimento está ilustrado na linha $t_{3}$ da Figura 5.8 na página 72 . O argumento desse procedimento é: 


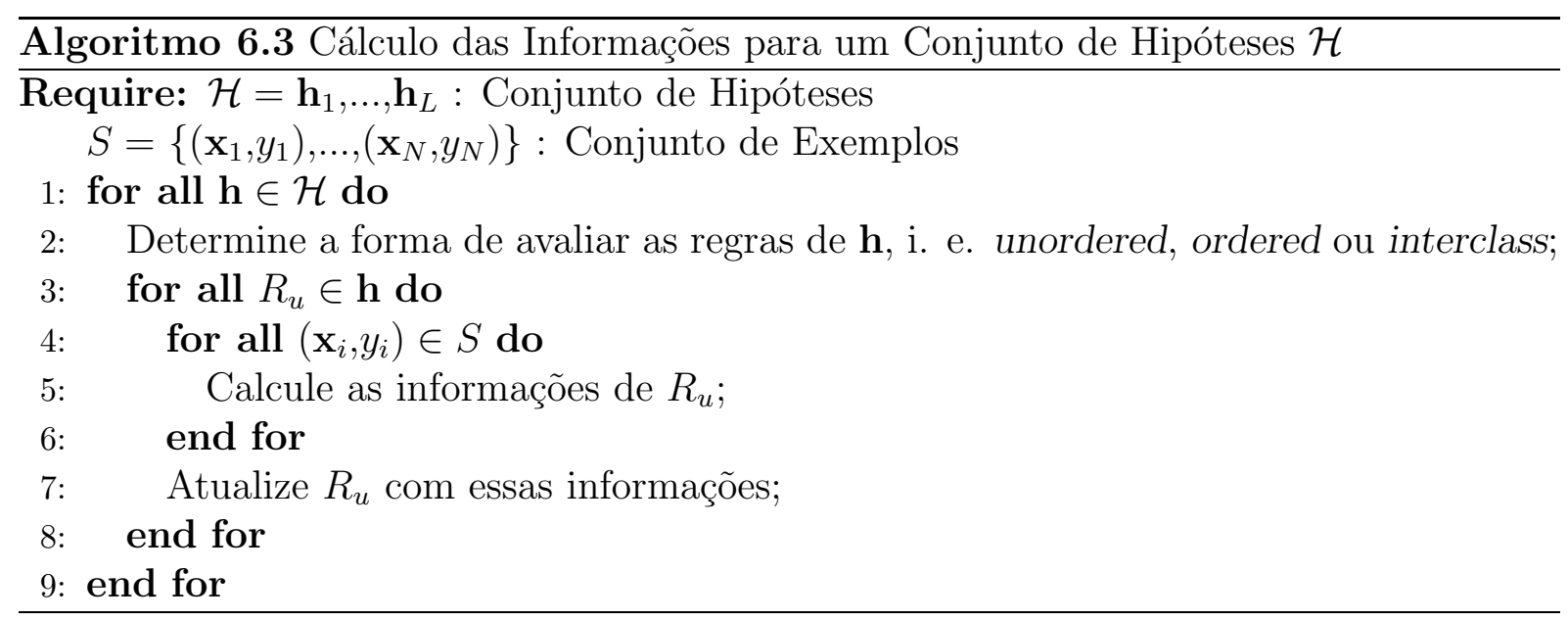

$+<$ arg-1 $>$ 'KNOW' calcula as medidas de avaliação de cada regra considerando apenas as informações referentes aos exemplos com valores conhecidos;

'UNKNOW' calcula as medidas de avaliação da regra considerando apenas as informações referentes aos exemplos com valores desconhecidos.

'ALL' calcula as medidas de avaliação da regra somando as informações referentes ao exemplos com valores conhecidos e desconhecidos.

As medidas de avaliação de cada regra são gravadas na Base de Fatos e podem ser referenciadas segundo os nomes na Tabela 6.1.

Ainda que todas as medidas relativas com peso sejam iguais entre si, e iguais à medida genérica Novidade (novR) — Seção 3.3.4, Teoremas 3.3.1 na página 37 e 3.3 .2 na página 40 - foi decidido manter a referência a essa medida utilizando os vários nomes freqüentemente usados na literatura para determiná-las.

Geralmente, medidas de avaliação são úteis para determinar quais são as "melhores" regras de um classificador simbólico h. As "melhores" regras podem ser aquelas que apresentam o maior (ou menor) valor para uma determinada medida de avaliação. Por exemplo, pode ser considerado que as "melhores" regras $R_{u}$ de um classificador simbólico h, são aquelas que possuem maior valor para a medida de Precisão (ou menor valor para a medida de Erro). 


\begin{tabular}{c|rl}
\hline \multirow{2}{*}{ Medidas } & accR: & Precisão da regra \\
errR: & Erro da regra \\
gegreln: & Confiança negativa da regra \\
sensR: & Sensitividade da regra \\
specR: & Especificidade da regra \\
& covR: & Cobertura da regra \\
supR: & Suporte da regra \\
& novR: & Novidade da regra \\
& satR: & Satisfação da regra \\
\hline \multirow{2}{*}{ Medidas } & raccR: & Precisão relativa da regra \\
relativas & rnegrelR: & Confiança negativa relativa da regra \\
& rsensR: & Sensitividade relativa da regra \\
& rspecR: & Especificidade relativa da regra \\
\hline \multirow{2}{*}{ MraccR: } & Precisão relativa com peso da regra \\
& wrnegrelR: & Confiança negativa relativa com peso da regra \\
relativas com peso & wrsensR: & Sensitividade relativa com peso da regra \\
& wrspecR: & Especificidade relativa com peso da regra \\
\hline
\end{tabular}

Tabela 6.1: Identificação das Medidas de Avaliação de Cada Regra

\subsection{Análise Quantitativa de Regras}

Sob o aspecto quantitativo regras são avaliadas com o intuito de selecionar aquelas que possam trazer algum conhecimento surpreendente ou inesperado para o usuário, ou seja, o objetivo dessas regras é selecionar as regras mais interessantes (Silberschatz and Tuzhilin, 1996). No Módulo de Análise de Regras a análise quantitativa é implementada por meio do cálculo das medidas objetivas Surpresa de Pequenos Disjuntos e Surpresa dos Atributos Individuais da Regra, ambas apresentadas no Seção 4.5, páginas 48 e 51, respectivamente.

Os procedimentos do MAR que possibilitam a análise quantitativa de regras, implementando medidas objetivas de interessabilidade, são:

1. Cálculo do Grau de Surpresa de Pequenos Disjuntos - smallDisjSurp/3

2. Cálculo do Grau de Surpresa dos Atributos Individuais da Regra - indAttSurp/2

O procedimento smallDisjSurp/3 está ilustrado na linha $t_{4}$ da Figura 5.8 na página 72. Considerando uma hipótese $\mathbf{h}$, esse procedimento encontra todas as regras $R_{u} \in \mathbf{h}$ cujo número de exemplos cobertos é menor ou igual ao valor $V t$ (pequenos disjuntos). Encontrados os pequenos disjuntos, o procedimento efetua os cálculos segundo a Equação 4.3 
na página 50. Como resultado, esse procedimento retorna uma lista contendo valores da medida de Surpresa dos Pequenos Disjuntos, inclusive já normalizados, para cada regra $R_{u} \in \mathbf{h}$. Os argumentos do procedimento smallDisjSurp/3 são:

$+<$ arg-1 $>$ identificador de uma hipótese;

$+<$ arg-2 $>$ valor do threshold;

- < arg-3 $>$ lista dos valores calculados do grau de surpresa dos pequenos disjuntos.

No Algoritmo 6.4 é descrito o cálculo do grau de Surpresa de Pequenos Disjuntos (SurpDisj e SurpDisjNorm) das regras de uma hipótese $\mathbf{h}$, dado um valor $V t$ para o treshold padrão.

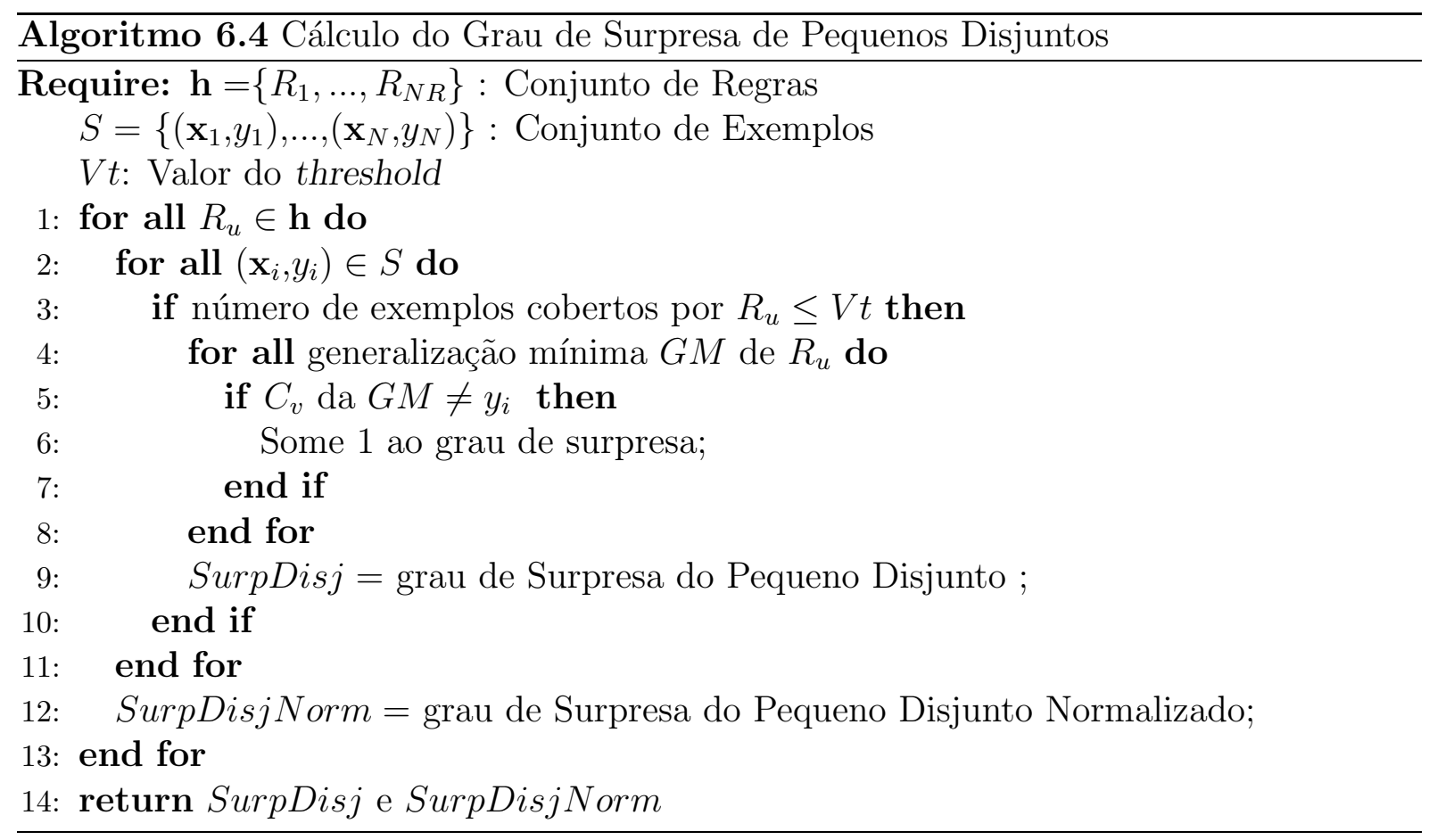

O cálculo do grau de Surpresa dos Atributos Individuais (discretos) da Regra é implementado pelo procedimento indAttSurp/2, ilustrado na linha $t_{2}$ da Figura 5.8.

Considerando uma hipótese h, o procedimento efetua os cálculos segundo a Equação 4.9 na página 53, gerando como resultado uma lista com os valores com o grau de Surpresa dos Atributos Individuais (discretos) de cada regra $R_{u} \in \mathbf{h}$.

Maiores detalhes a respeito do Módulo de Análise de Regras podem ser vistos em (Gomes and Monard, 2002a). Um exemplo de uso do MAR é apresentado a seguir. 


\subsection{Exemplo de Execução}

Nesta seção, um exemplo de execução do Módulo de Análise de Regras do $\mathcal{R}_{\text {ule }} \mathcal{S}_{\text {ystem }}$ é apresentado utilizando um conjunto de exemplos muito simples mostrado na Figura 6.2, e o conjunto de regras induzido pelo algoritmo de AM C 4.5 rules (Quinlan, 1993) ilustrado na Figura 6.3. Ambos conjuntos de exemplos e regras induzidas por $\mathcal{C} 4.5$ rules, encontramse na sintaxe padrão Prolog do $\mathcal{R}_{\text {ule }} \mathcal{S}_{\text {ystem }}$ (Gomes et al., 2002). Deve ser observado que o $\mathcal{C} 4.5$ rules induz regras interclass.

O conjunto de exemplos refere-se à medições diárias de condições meteorológicas, nas quais cada dia (exemplo) foi rotulado como "go" se as condições meteorológicas daquele dia são favoráveis para uma viagem à fazenda ou "dont_go", caso contrário.

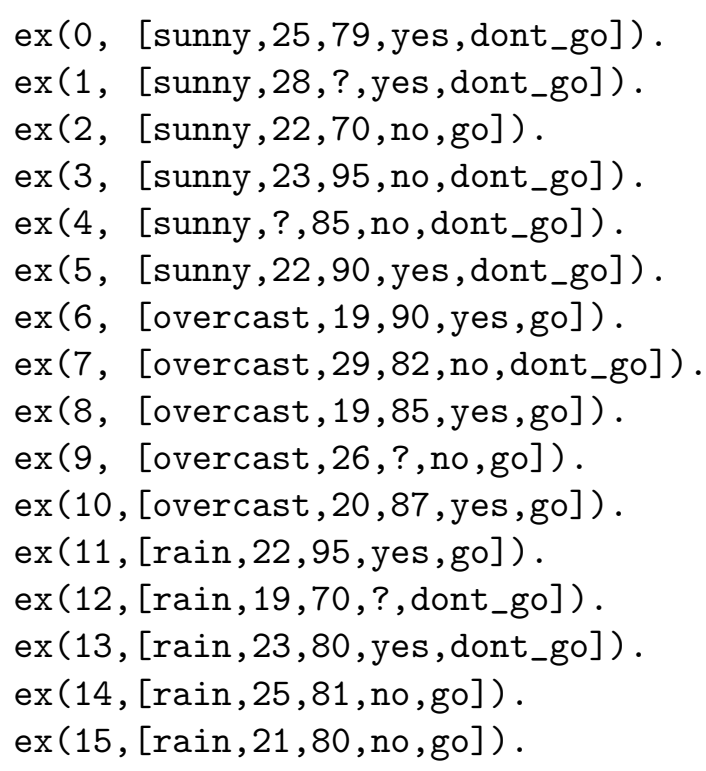

Figura 6.2: Arquivo de Exemplos

Como pode ser observado na Figura 5.8 na página 72 , partindo da linha $t_{0}$, os procedimentos loadRules/0 e loadExamples/0 irão carregar na Base de Fatos o arquivo de regras e o arquivo de exemplos, respectivamente.

$\mathrm{Na}$ linha $t_{1}$ o procedimento verifyBase/0 verifica se as regras e os exemplos na Base de Fatos pertencem ao mesmo domínio. Sendo bem sucedido, os procedimentos list0fExamples/1 e dictionary/1 são executados logo em seguida. 


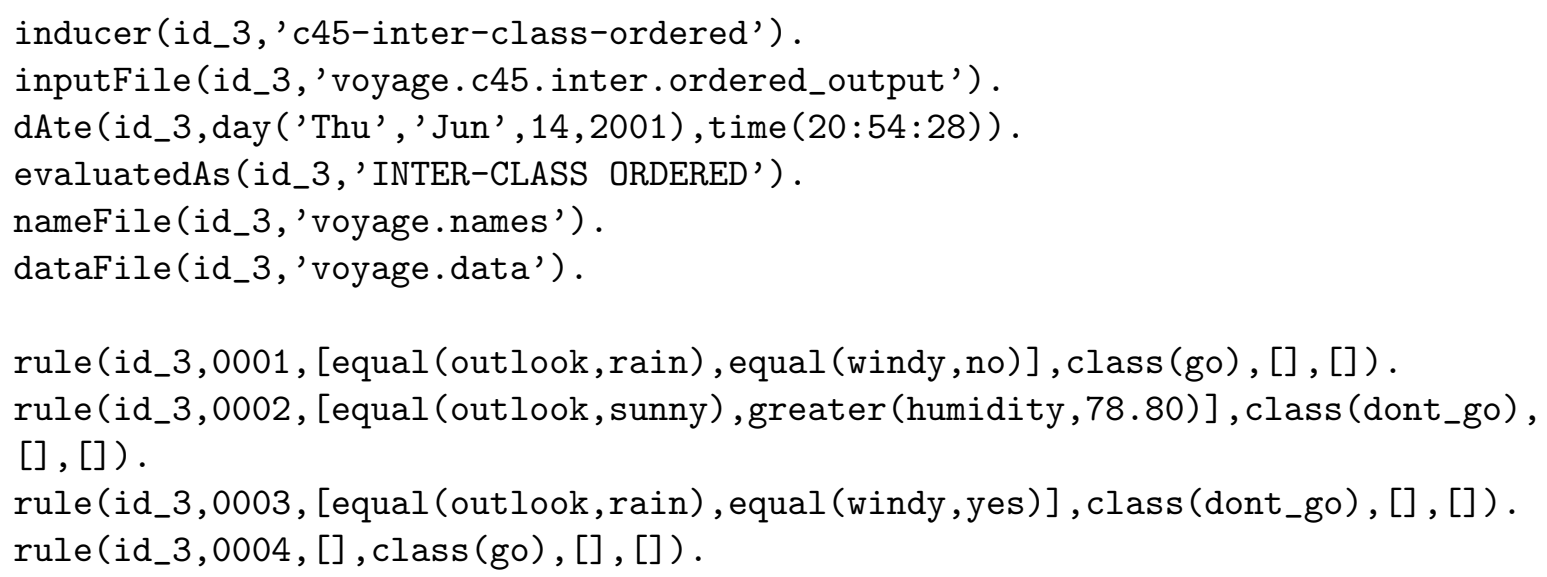

Figura 6.3: Arquivo de Regras Interclass Induzidas por $\mathcal{C} 4.5$ rules

$\mathrm{Na}$ linha $t_{2}$, o procedimento evaluateAllSetOfRuleFrequency/1 calcula os valores das freqüências relativas para o conjunto de regras armazenado na BF. Na Figura 6.4 são apresentados os resultados após a execução do procedimento.

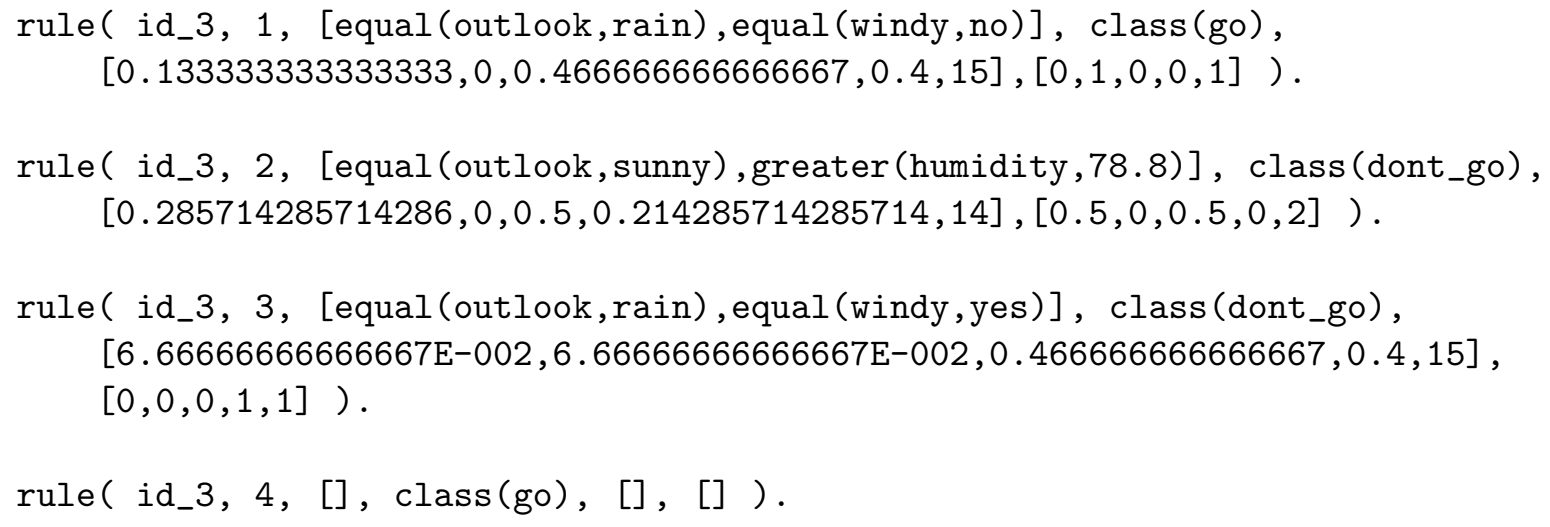

Figura 6.4: Regras na Base de Fatos após a Execução do Procedimento evaluateAllSetOfRuleFrequency/1

Ainda na linha $t_{2}$, o procedimento indAttSurp/2 calcula a medida de Interessabilidade dos Atributos Individuais de cada regra. Na Figura 6.5 são apresentados os valores encontrados dessa medida para cada regra.

$\mathrm{Na}$ linha $t_{3}$ da Figura 5.8 o procedimento calculateMeasuresOverAllSetOfRules/1 


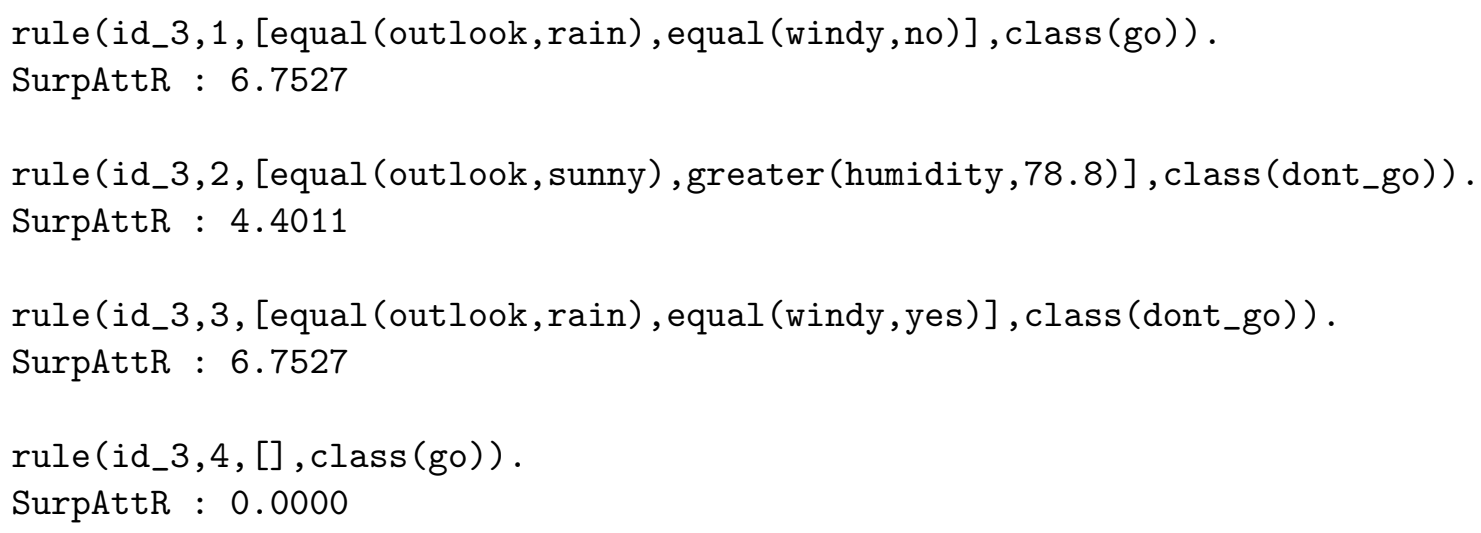

Figura 6.5: Surpresa dos Atributos Individuais das Regras

calcula as medidas qualitativas para cada regra, as quais são gravadas na Base de Fatos. Na Figura 6.6 são apresentados os valores dessas medidas para cada regra na BF. Esses valores foram calculados a partir da soma das informações referentes aos exemplos com valores conhecidos e desconhecidos. Deve ser observado que os valores foram pré-formatados para exibição no terminal de vídeo.

$\mathrm{Na}$ linha $t_{4}$ o procedimento smallDisjSurp/3 calcula o grau de surpresa dos pequenos disjuntos presentes na base. O treshold adotado para calcular essa medida é 0.20, ou seja, foram somente medidos os pequenos disjuntos que cobrem até $20 \%$ dos exemplos. Na Figura 6.7 são apresentados os pequenos disjuntos e os valores encontrados para essa medida.

Como já mencionado, vários conjuntos de regras pertencentes a diferentes classificadores simbólicos podem ser armazenados na Base de Fatos. Entretanto, é responsabilidade do usuário executar o procedimento verifyBase/0 para verificar que esses conjuntos de regras referem-se ao mesmo domínio do conjunto de exemplos (único) armazenado na BF.

\subsection{Considerações Finais}

Nesse capítulo foi descrito o Módulo de Análise de Regras do $\mathcal{R}_{\text {ule }} \mathcal{S}_{\text {ystem }}$ bem como os procedimentos que implementam as medidas de avaliação e de interessabilidade de regras, 


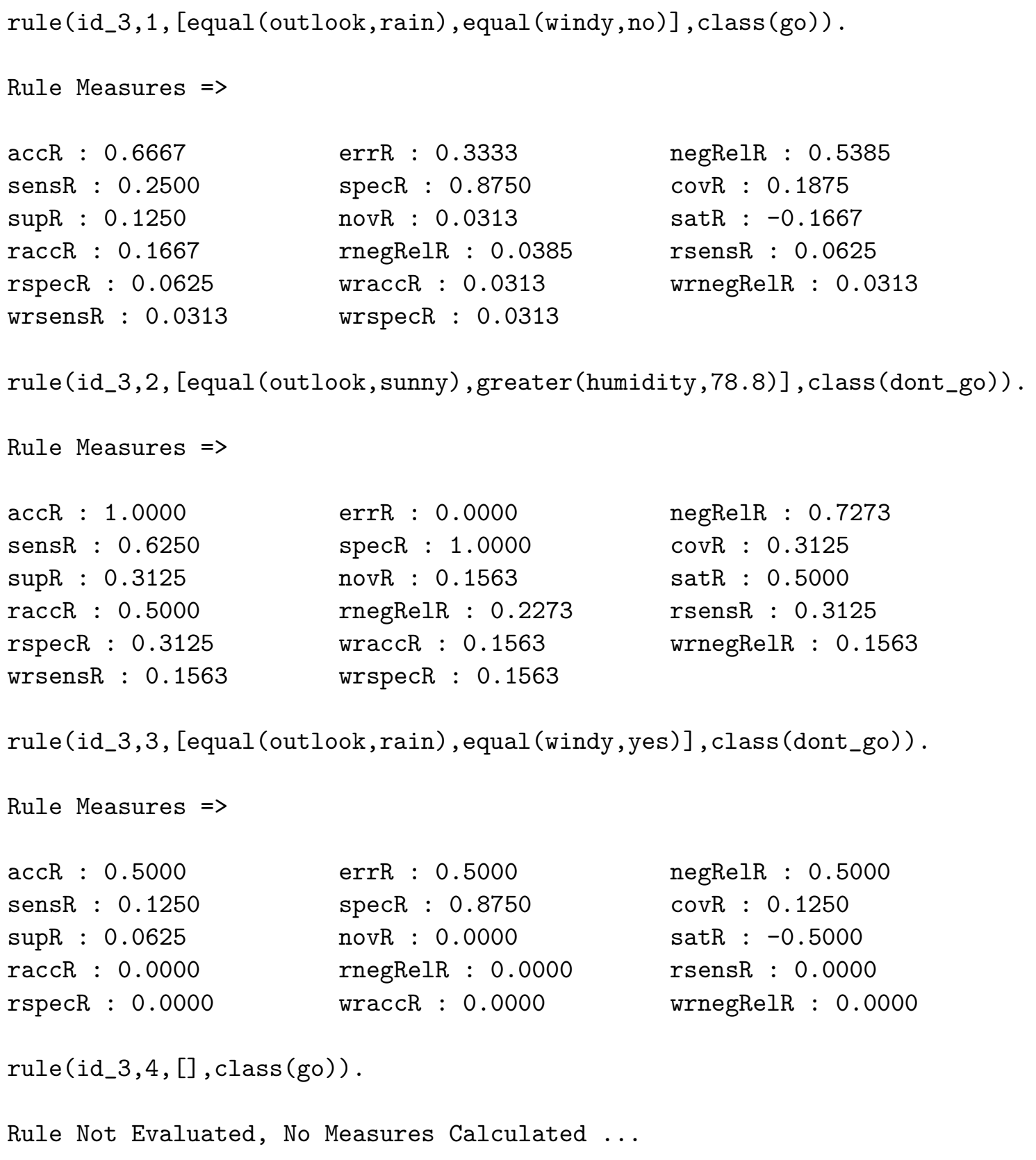

Figura 6.6: Medidas Qualitativas das Regras 


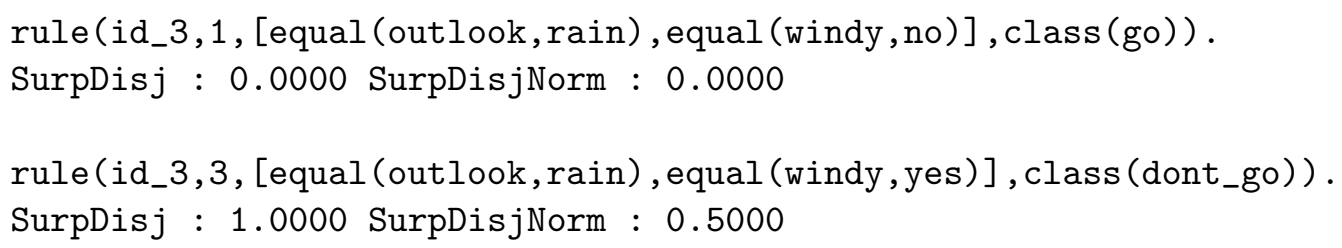

Figura 6.7: Surpresa dos Pequenos Disjuntos das Regras

apresentadas, respectivamente nos Capítulos 3 e 4. Ainda foi apresentado um exemplo de utilização desse módulo utilizando um conjunto de exemplos muito simples e um conjunto de regras induzido pelo algoritmo de $\mathrm{AM} \mathcal{C} 4.5$ rules.

Deve ser observado que, as informações calculadas pelo MAR, expressas sob a forma de estimativa de probabilidade (freqüências relativas) e posteriormente utilizadas para calcular as medidas de avaliação de regras, são expressas no MAR em um formato equivalente ao adotado no projeto Discover — Seção 5.3.1, página 59.

No próximo capítulo é apresentado um estudo de caso do mundo real, utilizando o MAR e diferentes classificadores simbólicos induzidos a partir desse conjunto de dados do mundo real. 


\section{Capítulo 7}

\section{Estudo de Caso Utilizando o Módulo de Análise de Regras do $\mathcal{R}_{\text {ule }} \mathcal{S}_{\text {ystem }}$}

\subsection{Considerações Iniciais}

O Módulo de Análise de Regras (MAR) do $\mathcal{R}_{\text {ule }} \mathcal{S}_{\text {ystem }}$, apresentado no capítulo anterior, tem como objetivo efetuar a análise qualitativa e quantitativa de regras induzidas por diferentes algoritmos de AM simbólico.

O estudo de caso a ser apresentado nesse capítulo tem por objetivo efetuar análise automática de regras através da execução dos procedimentos do Módulo de Análise de Regras. Essas regras compõem diferentes classificadores simbólicos, que são induzidos a partir de um conjunto de dados denominado Proc-a-gmg-d. Esse conjunto de dados refere-se ao processamento de sêmen diagnóstico.

A seguir, são feitos alguns comentários a respeito do processamento de sêmen diagnóstico e, logo em seguida, são apresentadas as etapas envolvidas nesse estudo de caso, bem como a discussão dos resultados obtidos.

\subsection{Processamento de Sêmen Diagnóstico}

O estudo de caso sobre processamento de sêmem diagnóstico teve seu início em (Lee, 2000), onde é descrita a importância desse processamento diagnóstico no tratamento para a reprodução assistida. A explicação a seguir está baseada nos trabalhos de Huei 
Diana Lee (Lee and Monard, 2000b; Lee and Monard, 2000a; Esteves et al., 2001) e foi desenvolvida de forma conjunta com Flávia Cristina Bernardini (Bernardini and Monard, 2002a). Esse exame permite:

1. Quantificar com precisão a qualidade do sêmen (processamento de sêmen diagnóstico);

2. Recuperar a maior quantidade possível de espermatozóides para a utilização na reprodução assistida (processamento de sêmen terapêutico).

A quantidade e aqualidade de espermatozóides recuperados pelo processamento de sêmen influencia na escolha da técnica que será utilizada no tratamento. São utilizadas três técnicas no tratamento para a reprodução assistida:

1. Inseminação Intra Uterina - IUI;

2. Fertilização In Vitro - FIV e

3. Injeção Intracitoplasmática do Espermatozóide no Oócito - ICSI.

Deve ser observado que o processamento de sêmen é bastante custoso. A realização do processamento de sêmen pode elevar o custo do exame em aproximadamente $80 \%$ do valor de um espermograma. Essa elevação de custo se deve principalmente a três fatores: necessidade de equipamentos especiais, mão de obra qualificada e tempo gasto para a realização do exame (Esteves and Bento, 1998; Esteves et al., 2000; Ferro et al., 2002). Assim, um dos interesses do estudo desse tema é tentar predizer qual será a quantidade de espermatozóides recuperados pelo processamento de sêmen antes mesmo da realização desse exame, a partir de exames menos custosos, como o espermograma. Dessa forma, dependendo da qualidade da predição, o especialista poderia decidir por uma técnica sem a necessidade da realização do processamento de sêmen. Um outro interesse para o estudo desse tema é a extração e avaliação do conhecimento adquirido.

O processamento de sêmen, através do fornecimento de melhores condições, permite que o maior número de espermatozóides móveis (motilidade) seja recuperado. Os espermatozóides são classificados em graus A, B, C e D dependendo de sua motilidade:

- grau A: espermatozóides que apresentam o maior grau de motilidade; 
- grau B: espermatozóides que apresentam um menor grau de motilidade;

- grau C: espermatozóides que se movem em círculos;

- grau D: espermatozóides que são imóveis.

Os graus de motilidade são atributos medidos tanto no espermograma quanto no processamento de sêmen. Os atributos medidos durante o processamento de sêmen são utilizados para a determinação das classes e os atributos medidos através do espermograma são fornecidos como atributos ao algoritmo de indução. Em outras palavras, os valores dos atributos de cada exemplo (paciente) são medidos utilizando um exame pouco custoso (espermograma, neste caso) enquanto que as classes desses exemplos são determinadas através de um outro exame mais custoso (processamento de sêmen, neste trabalho). A idéia é tentar verificar se é possível descobrir um relacionamento entre eles tal que, pelo menos para novos pacientes a necessidade de realizar o exame mais custoso seja minimizada. Este é um procedimento freqüentemente utilizado na área de medicina a fim de tentar diminuir, mas com segurança, o custo de um tratamento.

O especialista sugeriu duas possíveis opções para os valores considerados na classificação dos exemplos:

- A: considerar apenas a percentagem de espermatozóides classificados como de motilidade grau A no processamento de sêmen ou

- AB: considerar a percentagem de espermatozóides classificados como de motilidade grau A e grau B no processamento de sêmen.

Assim, foram definidas três classes, baseadas nas técnicas que são utilizadas no tratamento para a reprodução assistida:

- 1: $x<1$ - IUI;

- 2: $1 \leq x<5-$ FIV e

- 3: $x \geq 5$ - ICSI.

onde $x$ representa milhões de espermatozóides por mililitro (ml).

Os casos para a composição desses conjunto de dados foram extraídos de casos reais do Centro de Fertilização, em Campinas, SP fornecidos pelo especialista Dr. Sandro 
Esteves. Em (Lee, 2000), foram calculados para cada caso os valores A e AB, gerando dois conjuntos de dados, Proc-a e Proc-ab, respectivamente, cada um com 231 exemplos. Diversos experimentos foram conduzidos considerando-se esses dois conjuntos separadamente. Com o decorrer do trabalho, necessitou-se criar novos atributos como combinação de atributos originais, criando-se diferentes conjuntos de dados (Lee and Monard, 2000b). Um desses conjunto de dados que mostrou um bom resultado com relação à predição dos classificadores induzidos foi o denominado Proc-a-gmg-d, o qual considera somente os exemplos em que o processamento de sêmen é diagnóstico.

Posteriormente, o conjunto de exemplos Proc-a do trabalho inicial foram acrescentados novos casos. Tomando-se desse conjunto de exemplos somente os exemplos em que o processamento de sêmen é diagnóstico, foi obtido um segundo conjunto de exemplos Proc-a-gmg-d,semelhante ao construído com os exemplos Proc-a do trabalho inicial, mas incluindo os novos casos, o qual é utilizado neste trabalho. Deve ser observado que todo o trabalho de coleta, preparação e limpeza dos dados foi realizada por Huei Diana Lee.

\subsection{Etapas do Estudo de Caso}

Conforme mencionado anteriormente, o objetivo do estudo apresentado neste trabalho é efetuar a análise qualitativa e quantitativa das regras induzidas utilizando os dados descritos na Seção 7.3.1, através da execução dos procedimentos do Módulo de Análise de Regras do $\mathcal{R}_{\text {ule }} \mathcal{S}_{\text {ystem. }}$. Assim, vários conjuntos de regras, utilizando diferentes algoritmos de AM, foram induzidos a partir do conjunto de dados Proc-a-gmg-d.

As etapas envolvidas nesse estudo são:

- Descrição e preparação do conjunto de dados;

- Indução e preparação dos conjuntos de regras induzidas por diferentes algoritmos de AM;

- Análise quantitativa dos conjuntos de regras; e

- Análise qualitativa dos conjuntos de regras. 


\subsubsection{Descrição e Preparação do Conjunto de Dados}

A Tabela 7.1 sumariza as características do conjunto de dados utilizado neste estudo. Ela mostra o número de exemplos (\#Exemplos), número e porcentagem de exemplos com valores duplicados (que aparecem mais de uma vez) ou conflitantes (que possuem o mesmo atributo-valor mas têm diferentes classificações), número de atributos (\#Atributos) contínuos e nominais incluindo atributo classificatório, distribuição de classes, o erro majoritário e se o conjunto de dados tem ao menos um valor desconhecido(?) ${ }^{1}$. Na Tabela 7.2 são descritos os atributos que compõem o conjunto de dados Proc-a-gmg-d.

\begin{tabular}{c|cc|c|cc|c|c}
\hline \hline $\begin{array}{c}\text { Conjunto } \\
\text { de Dados }\end{array}$ & \# Exemplos & $\begin{array}{c}\text { \# Duplicados ou } \\
\text { Conflitantes (\%) }\end{array}$ & $\begin{array}{c}\text { \# Atributos } \\
\text { (cont.,nom.) }\end{array}$ & Classes & Classe \% & Erro CM & $?$ \\
\hline Proc-a-gmg-d & 240 & $1(0.416 \%)$ & $22(17,5)$ & um & $42.92 \%$ & $57.08 \%$ & Sim \\
& & & & dois & $22.08 \%$ & sobre um & \\
& & & & tres & $35.00 \%$ & & \\
\hline \hline
\end{tabular}

Tabela 7.1: Sumário das Características do Conjunto de Dados Proc-a-gmg-d

O conjunto de dados Proc-a-gmg-d é utilizado tanto na indução dos classificadores simbólicos quanto na análise dos conjuntos de regras realizada pelo MAR.

Para que sejam executados os procedimentos implementados no MAR, o formato do conjunto de dados Proc-a-gmg-d deve, primeiramente, ser convertido para a sintaxe padrão Prolog do $\mathcal{R}_{\text {ule }} \mathcal{S}_{\text {ystem }}$ (Gomes et al., 2002).

\subsubsection{Indução e Preparação dos Conjuntos de Regras}

Foram induzidos dois classificadores simbólicos a partir do conjunto de dados Proca-gmg-d. Utilizando o algoritmo de AM $\mathcal{C N} 2$ (Clark and Niblett, 1989) foi induzido um conjunto de regras unordered $-\mathbf{h}_{1}$. Utilizando o algoritmo $\mathcal{S e e} 5^{2}$ foi induzido um conjunto de regras interclass $-\mathbf{h}_{2}$.

Logo após, cada regra de cada classificador foram enumeradas e, utilizando o conversor descrito em (Gomes et al., 2002), ambos conjuntos de regras foram convertidos para a sintaxe padrão Prolog do $\mathcal{R}_{\text {ule }} \mathcal{S}_{\text {ystem }}$. Nessa conversão automática, cada regra do classificador $\mathbf{h}_{1}$ passou a ser identificada por id_105 e as do classificador $\mathbf{h}_{2}$ por id_104. Os

\footnotetext{
${ }^{1}$ Essas informações foram obtidas utilizando a ferramenta info da $\mathcal{M L C}++$.

${ }^{2}$ http://www.rulequest.com
} 


\begin{tabular}{|c|c|c|c|c|c|}
\hline \multirow{2}{*}{$\begin{array}{l}\text { Número da } \\
\text { Feature }\end{array}$} & \multirow{2}{*}{$\begin{array}{l}\text { Nome da } \\
\text { Feature }\end{array}$} & \multirow[t]{2}{*}{ Descrição } & \multicolumn{3}{|c|}{ \#Valores Distintos } \\
\hline & & & possíveis & atuais & tipo \\
\hline$\# 0$ & idade & Idade do paciente & - & 26 & contínuo \\
\hline$\# 1$ & hora & Período da coleta da amostra de sêmen & & & \\
\hline & & 1: manhã; $2:$ tarde & 2 & 3 & discreto \\
\hline$\# 2$ & processamento & Processamento do sêmen realizado após & & & \\
\hline & & esta quantidade de minutos após a coleta & - & 12 & contínuo \\
\hline$\# 3$ & tempo-abs & Tempo de abstinência & - & 12 & contínuo \\
\hline$\# 4$ & volume & Volume de sêmen coletado & - & 55 & contínuo \\
\hline$\# 5$ & cor & Cor do sêmen coletado & & & \\
\hline$\# 6$ & odor & $\begin{array}{l}\text { 1: branco-opalescente (normal); } \\
\text { 2: amarelo-opalescente; } 3 \text { : translúcido } \\
\text { Odor do sêmen coletado }\end{array}$ & 3 & 4 & discreto \\
\hline & & 1: característico; 2 : forte; $3:$ urina & 3 & 3 & discreto \\
\hline$\# 7$ & $\mathrm{pH}$ & pH do sêmen coletado & - & 13 & contínuo \\
\hline$\# 8$ & viscosidade & Viscosidade do sêmen coletado & & & \\
\hline & & 1: normal; 2 : aumentada & 2 & 2 & discreto \\
\hline$\# 9$ & liquefacao & Liquefação do sêmen coletado & & & \\
\hline & & 1: completa; 2 : imconpleta & 2 & 2 & discreto \\
\hline$\# 10$ & concentracao & $\begin{array}{l}\text { Concentração de espermatozóides } \\
\text { por ml coletado }\end{array}$ & - & 198 & contínuo \\
\hline \#11 & concentracao-total & Concentração total de espermatozóides & - & 219 & contínuo \\
\hline const \#12 & motilidade & \% de espermatozóides móveis & - & 74 & contínuo \\
\hline \#13 & class-A & Classificação da motilidade - Grau A & - & 66 & contínuo \\
\hline \#14 & class-B & Grau B & - & 53 & contínuo \\
\hline$\# 15$ & class-C & Grau C & - & 44 & contínuo \\
\hline \#16 & class-D & Grau D & - & 76 & contínuo \\
\hline \#17 & vitalidade & \% de espermatozóides vivos & - & 59 & contínuo \\
\hline \#18 & num-leu & Número de leucócitos & - & 66 & contínuo \\
\hline \#19 & Kruger & Morfologia Estrita de Kruger & & & \\
\hline & & - \% de formas normais & - & 28 & contínuo \\
\hline$\# 20$ & HP & Teste Hipo-osmótico - \% de inchados & - & 54 & contínuo \\
\hline
\end{tabular}

Tabela 7.2: Descrição dos Atributos do Conjunto de Dados Proc-a-gmg-d 
conjuntos de regras $\mathbf{h}_{1}$ e $\mathbf{h}_{2}$ podem ser vistos em detalhes, inclusive descritos na sintaxe padrão Prolog, em (Gomes and Monard, 2002b).

Além de induzir as hipóteses $\mathbf{h}_{1}$ e $\mathbf{h}_{2}$ utilizando todos os exemplos disponíveis (240 exemplos) foi também estimado o erro de $\mathbf{h}_{1}$ e $\mathbf{h}_{2}$ utilizando a técnica 10-fold-crossvalidation. A Tabela 7.3 mostra os resultados obtidos.

\begin{tabular}{|c|c|c|c|c|c|}
\hline \hline $\begin{array}{c}\text { Indutor } \\
\text { Utilizado }\end{array}$ & Classificador & $\begin{array}{c}\text { Número Regras } \\
\text { Induzidas }^{a}\end{array}$ & $\begin{array}{c}\text { Forma de Indução } \\
\text { das Regras }\end{array}$ & $\begin{array}{c}\text { Erro } \\
\text { Aparente }\end{array}$ & $\begin{array}{c}\text { Erro } \pm \text { Desvio } \\
\text { Padrão }\end{array}$ \\
\hline $\mathcal{C N} 2$ & $\mathbf{h}_{1}$ & 32 & unordered & $12,9 \%$ & $41.66 \% \pm 5.13 \%$ \\
\hline $\mathcal{S}$ ee5 & $\mathbf{h}_{2}$ & 19 & interclass & $15,4 \%$ & $44.20 \% \pm 2.90 \%$ \\
\hline
\end{tabular}

${ }^{a} \mathrm{Na}$ contagem do número de regras não foi considerada a regra default.

Tabela 7.3: Informações sobre os Conjuntos de Regras Induzidas

Como pode ser observado, a estimativa de erro de cada classificador em novos exemplos ainda não vistos, i.e., $41.66 \% \pm 5.13 \%$ para $\mathbf{h}_{1}$ e $44.20 \% \pm 2.90 \%$ para $\mathbf{h}_{2}$, ainda que inferior ao erro da classe majoritária ( $57.08 \%$ na Tabela 7.1 ) é muito alto para a área médica. Isso significa que $\mathbf{h}_{1}$ e $\mathbf{h}_{2}$, considerados como caixa preta, não são apropriadas para classificar (rotular) qualquer outro novo caso desse domínio.

Entretanto, analisando individualmente as regras que constituem cada classificador, existe a possibilidade de encontrar, por exemplo, algumas regras apropriadas para classificar com bastante confiança alguns desses novos casos.

\subsubsection{Análise Qualitativa dos Conjuntos de Regras}

Para efetuar a análise qualitativa dos conjuntos de regras $\mathbf{h}_{1}$ e $\mathbf{h}_{2}$ foram executados os seguintes procedimentos do MAR:

1. Cálculo de Informações de Regras - evaluateAllSetOfRuleFrequency/1.

2. Cálculo de Medidas de Avaliação de Regras - calculateMeasuresOverAllSetOFRules/1.

Ao ser executado o procedimento evaluateAllSetOfRuleFrequency/1 as informações $f_{h b}, f_{\bar{h} b}, f_{\overline{h b}}, f_{h \bar{b}}$, referentes aos exemplos com valores conhecidos e desconhecidos, são calculadas e adicionadas ao final de cada regra de $\mathbf{h}_{1} \mathbf{e} \mathbf{h}_{2}$. 
Deve ser ressaltado que essas informações, ao serem calculadas para as regras de $\mathbf{h}_{1}$, diferem um pouco dos valores apresentados no classificador equivalente induzido pelo algoritmo $\mathcal{C N}$. Isso deve-se ao fato de que, se o exemplo apresentar atributos com valores desconhecidos, ele sempre será contado como verdade no cálculo das informações realizado pelo MAR. Em outras palavras o procedimento evaluateAllSetOfRuleFrequency/1 sempre conta 1 para $B$ e/ou $H$ verdade para esse exemplo. Entretanto, o $\mathcal{C N} 2$ trata esse caso contando 0.5 para cada exemplo com valores desconhecidos, coberto por uma regra.

Prosseguindo com a análise qualitativa desses dois conjuntos de regras, foram calculadas medidas de avaliação por meio da execução do procedimento calculateMeasuresOverAllSetOFRules/1. Os valores das medidas genéricas encontradas para as regras de $\mathbf{h}_{1}$ e $\mathbf{h}_{2}$ são apresentados, respectivamente, nas Tabela 7.4 e 7.5. As medidas relativas, com e sem peso, calculadas para as regras de $\mathbf{h}_{1}$ e $\mathbf{h}_{2}$, são apresentadas, respectivamente, nas Tabelas 7.6 e 7.7. Para melhor compreensão, em cada uma dessa tabelas são apresentados os valores $h b, \bar{h} b, \overline{h b}, h \bar{b}$ utilizados no cálculo das freqüências $f_{h b}, f_{\bar{h} b}, f_{\overline{h b}}, f_{h \bar{b}}$.

\subsubsection{Análise Quantitativa dos Conjuntos de Regras}

Para efetuar a análise quantitativa de regras dos conjuntos de regras $\mathbf{h}_{1}$ e $\mathbf{h}_{2}$ foram executados os seguintes procedimentos do MAR:

1. Cálculo do Grau de Surpresa de Pequenos Disjuntos - smallDisjSurp/3

2. Cálculo do Grau de Surpresa dos Atributos Individuais da Regra - indAttSurp/2

De acordo com a medida de Surpresa de Pequenos Disjuntos, nas Figura 7.1 e 7.2 são apresentados, respectivamente, em ordem crescente dos valores calculados pelo $\mathrm{MAR}^{3}$, os pequenos disjuntos que fazem parte dos conjuntos de regras $\mathbf{h}_{1} \mathbf{e} \mathbf{h}_{2}$. Esses valores foram obtidos admitindo-se, como critério para determinar quais regras são pequenos disjuntos, o número de exemplos cobertos pela regra. No caso aqui apresentado, pequenos disjuntos são regras que cobrem no máximo $10 \%$ dos exemplos (ou seja, regras com $\operatorname{Cov}(R) \leq 0.10$ ).

De acordo com a medida de Surpresa dos Atributos Individuais Discretos, as Figura 7.3 e 7.4 mostram, respectivamente, as regras selecionadas do conjunto de regras $\mathbf{h}_{1}$ e $\mathbf{h}_{2}$.

\footnotetext{
${ }^{3}$ Foram desconsiderados pequenos disjuntos com grau de surpresa igual a 0 (zero).
} 


\begin{tabular}{|c|c|c|c|c|c|c|c|}
\hline \multirow{2}{*}{$\begin{array}{l}\# \text { da } \\
\text { Regra }\end{array}$} & \multicolumn{6}{|c|}{ Medidas Genéricas } & \multirow{2}{*}{$\begin{array}{l}\text { Valores Absolutos } \\
{[h b, \bar{h} b, h \bar{b}, \overline{h b}, N]}\end{array}$} \\
\hline & $\mathrm{accR}$ & negrelR & sensR & covR & supR & novR & \\
\hline 1 & 1.0000 & 0.6587 & 0.3107 & 0.1333 & 0.1333 & 0.0761 & {$[32,0,71,137,240]$} \\
\hline 2 & 1.0000 & 0.6343 & 0.2330 & 0.1000 & 0.1000 & 0.0571 & {$[24,0,79,137,240]$} \\
\hline 3 & 1.0000 & 0.6143 & 0.1650 & 0.0708 & 0.0708 & 0.0404 & {$[17,0,86,137,240]$} \\
\hline 4 & 1.0000 & 0.6313 & 0.2233 & 0.0958 & 0.0958 & 0.0547 & {$[23,0,80,137,240]$} \\
\hline 5 & 1.0000 & 0.6116 & 0.1553 & 0.0667 & 0.0667 & 0.0381 & {$[16,0,87,137,240]$} \\
\hline 6 & 1.0000 & 0.6524 & 0.2913 & 0.1250 & 0.1250 & 0.0714 & {$[30,0,73,137,240]$} \\
\hline 7 & 1.0000 & 0.5905 & 0.0777 & 0.0333 & 0.0333 & 0.0190 & {$[8,0,95,137,240]$} \\
\hline 8 & 1.0000 & 0.5880 & 0.0680 & 0.0292 & 0.0292 & 0.0166 & $7,0,96,137,240]$ \\
\hline 9 & 1.0000 & 0.5805 & 0.0388 & 0.0167 & 0.0167 & 0.0095 & $4,0,99,137,240]$ \\
\hline 10 & 1.0000 & 0.6171 & 0.1748 & 0.0750 & 0.0750 & 0.0428 & $18,0,85,137,240$ \\
\hline 11 & 1.0000 & 0.5732 & 0.0097 & 0.0042 & 0.0042 & 0.0024 & {$[1,0,102,137,240]$} \\
\hline 12 & 1.0000 & 0.8026 & 0.1321 & 0.0292 & 0.0292 & 0.0227 & {$[7,0,46,187,240]$} \\
\hline 13 & 1.0000 & 0.8095 & 0.1698 & 0.0375 & 0.0375 & 0.0292 & $9,0,44,187,240]$ \\
\hline 14 & 1.0000 & 0.7890 & 0.0566 & 0.0125 & 0.0125 & 0.0097 & $3,0,50,187,240]$ \\
\hline 15 & 1.0000 & 0.7857 & 0.0377 & 0.0083 & 0.0083 & 0.0065 & $2,0,51,187,240]$ \\
\hline 16 & 1.0000 & 0.7857 & 0.0377 & 0.0083 & 0.0083 & 0.0065 & $2,0,51,187,240]$ \\
\hline 17 & 1.0000 & 0.8026 & 0.1321 & 0.0292 & 0.0292 & 0.0227 & $7,0,46,187,240]$ \\
\hline 18 & 1.0000 & 0.7824 & 0.0189 & 0.0042 & 0.0042 & 0.0032 & $1,0,52,187,240]$ \\
\hline 19 & 1.0000 & 0.7824 & 0.0189 & 0.0042 & 0.0042 & 0.0032 & $1,0,52,187,240]$ \\
\hline 20 & 1.0000 & 0.7824 & 0.0189 & 0.0042 & 0.0042 & 0.0032 & {$[1,0,52,187,240]$} \\
\hline 21 & 1.0000 & 0.6996 & 0.2024 & 0.0708 & 0.0708 & 0.0460 & {$[17,0,67,156,240]$} \\
\hline 22 & 1.0000 & 0.6964 & 0.1905 & 0.0667 & 0.0667 & 0.0433 & {$[16,0,68,156,240]$} \\
\hline 23 & 1.0000 & 0.7027 & 0.2143 & 0.0750 & 0.0750 & 0.0488 & {$[18,0,66,156,240]$} \\
\hline 24 & 1.0000 & 0.7091 & 0.2381 & 0.0833 & 0.0833 & 0.0542 & {$[20,0,64,156,240]$} \\
\hline 25 & 1.0000 & 0.6933 & 0.1786 & 0.0625 & 0.0625 & 0.0406 & {$[15,0,69,156,240]$} \\
\hline 26 & 1.0000 & 0.6933 & 0.1786 & 0.0625 & 0.0625 & 0.0406 & {$[15,0,69,156,240]$} \\
\hline 27 & 1.0000 & 0.6610 & 0.0476 & 0.0167 & 0.0167 & 0.0108 & {$[4,0,80,156,240]$} \\
\hline 28 & 1.0000 & 0.6582 & 0.0357 & 0.0125 & 0.0125 & 0.0081 & $3,0,81,156,240]$ \\
\hline 29 & 1.0000 & 0.6638 & 0.0595 & 0.0208 & 0.0208 & 0.0135 & $5,0,79,156,240]$ \\
\hline 30 & 1.0000 & 0.6527 & 0.0119 & 0.0042 & 0.0042 & 0.0027 & $1,0,83,156,240]$ \\
\hline 31 & 1.0000 & 0.6667 & 0.0714 & 0.0250 & 0.0250 & 0.0163 & {$[6,0,78,156,240]$} \\
\hline 32 & 1.0000 & 0.6783 & 0.1190 & 0.0417 & 0.0417 & 0.0271 & {$[10,0,74,156,240]$} \\
\hline
\end{tabular}

Tabela 7.4: Valores das Medidas Genéricas para as Regras de $\mathbf{h}_{1}$

\begin{tabular}{|c|c|c|c|c|c|c|c|c|c|c|}
\hline \hline \multirow{2}{*}{$\begin{array}{c}\text { \# da } \\
\text { Regra }\end{array}$} & \multicolumn{9}{|c|}{ Medidas Genéricas } & $\begin{array}{c}\text { Valores Absolutos } \\
{[}\end{array}$ \\
\cline { 2 - 11 } & accR & errR & negrelR & sensR & specR & covR & supR & novR & satR & $\overline{h b}, N]$ \\
\hline 1 & 0.9111 & 0.0889 & 0.6821 & 0.3981 & 0.9708 & 0.1875 & 0.1708 & 0.0904 & 0.8443 & {$[41,4,62,133,240]$} \\
\hline 2 & 1.0000 & 0.0000 & 0.5957 & 0.0971 & 1.0000 & 0.0417 & 0.0417 & 0.0238 & 1.0000 & {$[10,0,93,137,240]$} \\
\hline 3 & 1.0000 & 0.0000 & 0.5931 & 0.0874 & 1.0000 & 0.0375 & 0.0375 & 0.0214 & 1.0000 & {$[9,0,94,137,240]$} \\
\hline 4 & 0.8800 & 0.1200 & 0.6233 & 0.2136 & 0.9781 & 0.1042 & 0.0917 & 0.0470 & 0.7898 & {$[22,3,81,134,240]$} \\
\hline 5 & 0.8493 & 0.1507 & 0.7545 & 0.6019 & 0.9197 & 0.3042 & 0.2583 & 0.1278 & 0.7360 & {$[62,11,41,126,240]$} \\
\hline 6 & 1.0000 & 0.0000 & 0.5805 & 0.0388 & 1.0000 & 0.0167 & 0.0167 & 0.0095 & 1.0000 & {$[4,0,99,137,240]$} \\
\hline 7 & 0.8750 & 0.1250 & 0.5862 & 0.0680 & 0.9927 & 0.0333 & 0.0292 & 0.0149 & 0.7810 & {$[7,1,96,136,240]$} \\
\hline 8 & 0.8125 & 0.1875 & 0.5982 & 0.1262 & 0.9781 & 0.0667 & 0.0542 & 0.0256 & 0.6715 & {$[13,3,90,134,240]$} \\
\hline 9 & 1.0000 & 0.0000 & 0.7991 & 0.1132 & 1.0000 & 0.0250 & 0.0250 & 0.0195 & 1.0000 & {$[6,0,47,187,240]$} \\
\hline 10 & 1.0000 & 0.0000 & 0.7957 & 0.0943 & 1.0000 & 0.0208 & 0.0208 & 0.0162 & 1.0000 & {$[5,0,48,187,240]$} \\
\hline 11 & 0.8571 & 0.1429 & 0.7983 & 0.1132 & 0.9947 & 0.0292 & 0.0250 & 0.0186 & 0.8167 & {$[6,1,47,186,240]$} \\
\hline 12 & 1.0000 & 0.0000 & 0.7824 & 0.0189 & 1.0000 & 0.0042 & 0.0042 & 0.0032 & 1.0000 & {$[1,0,52,187,240]$} \\
\hline 13 & 0.8000 & 0.2000 & 0.7915 & 0.0755 & 0.9947 & 0.0208 & 0.0167 & 0.0121 & 0.7433 & {$[4,1,49,186,240]$} \\
\hline 14 & 0.7500 & 0.2500 & 0.7881 & 0.0566 & 0.9947 & 0.0167 & 0.0125 & 0.0088 & 0.6791 & {$[3,1,50,186,240]$} \\
\hline 15 & 0.7500 & 0.2500 & 0.7881 & 0.0566 & 0.9947 & 0.0167 & 0.0125 & 0.0088 & 0.6791 & {$[3,1,50,186,240]$} \\
\hline 16 & 1.0000 & 0.0000 & 0.7857 & 0.0377 & 1.0000 & 0.0083 & 0.0083 & 0.0065 & 1.0000 & {$[2,0,51,187,240]$} \\
\hline 17 & 1.0000 & 0.0000 & 0.7027 & 0.2143 & 1.0000 & 0.0750 & 0.0750 & 0.0488 & 1.0000 & {$[18,0,66,156,240]$} \\
\hline 18 & 0.8571 & 0.1429 & 0.7576 & 0.4286 & 0.9615 & 0.1750 & 0.1500 & 0.0888 & 0.7802 & {$[36,6,48,150,240]$} \\
\hline 19 & 0.8161 & 0.1839 & 0.9150 & 0.8452 & 0.8974 & 0.3625 & 0.2958 & 0.1690 & 0.7171 & {$[71,16,13,140,240]$} \\
\hline \hline
\end{tabular}

Tabela 7.5: Valores das Medidas Genéricas para as Regras de $\mathbf{h}_{2}$ 


\begin{tabular}{|c|c|c|c|c|c|c|}
\hline \multirow{2}{*}{$\begin{array}{l}\text { \# da } \\
\text { Regra }\end{array}$} & \multicolumn{5}{|c|}{ Medidas Relativas } & \multirow{2}{*}{$\begin{array}{l}\text { Valores Absolutos } \\
{[h b, \bar{h} b, h \bar{b}, \overline{h b}, N]}\end{array}$} \\
\hline & raccR & rnegrelR & rsensR & rspecR & wraccR $^{a}$ & \\
\hline 1 & 0.5708 & 0.0878 & 0.1773 & 0.1333 & 0.0761 & {$[32,0,71,137,240]$} \\
\hline 2 & 0.5708 & 0.0634 & 0.1330 & 0.1000 & 0.0571 & {$[24,0,79,137,240]$} \\
\hline 3 & 0.5708 & 0.0435 & 0.0942 & 0.0708 & 0.0404 & {$[17,0,86,137,240]$} \\
\hline 4 & 0.5708 & 0.0605 & 0.1275 & 0.0958 & 0.0547 & {$[23,0,80,137,240]$} \\
\hline 5 & 0.5708 & 0.0408 & 0.0887 & 0.0667 & 0.0381 & {$[16,0,87,137,240]$} \\
\hline 6 & 0.5708 & 0.0815 & 0.1663 & 0.1250 & 0.0714 & {$[30,0,73,137,240]$} \\
\hline 7 & 0.5708 & 0.0197 & 0.0443 & 0.0333 & 0.0190 & {$[8,0,95,137,240]$} \\
\hline 8 & 0.5708 & 0.0171 & 0.0388 & 0.0292 & 0.0166 & $7,0,96,137,240]$ \\
\hline 9 & 0.5708 & 0.0097 & 0.0222 & 0.0167 & 0.0095 & $4,0,99,137,240]$ \\
\hline 10 & 0.5708 & 0.0463 & 0.0998 & 0.0750 & 0.0428 & $18,0,85,137,240]$ \\
\hline 11 & 0.5708 & 0.0024 & 0.0055 & 0.0042 & 0.0024 & {$[1,0,102,137,240]$} \\
\hline 12 & 0.7792 & 0.0234 & 0.1029 & 0.0292 & 0.0227 & {$[7,0,46,187,240]$} \\
\hline 13 & 0.7792 & 0.0304 & 0.1323 & 0.0375 & 0.0292 & $9,0,44,187,240]$ \\
\hline 14 & 0.7792 & 0.0099 & 0.0441 & 0.0125 & 0.0097 & $3,0,50,187,240]$ \\
\hline 15 & 0.7792 & 0.0065 & 0.0294 & 0.0083 & 0.0065 & $2,0,51,187,240]$ \\
\hline 16 & 0.7792 & 0.0065 & 0.0294 & 0.0083 & 0.0065 & $2,0,51,187,240]$ \\
\hline 17 & 0.7792 & 0.0234 & 0.1029 & 0.0292 & 0.0227 & $7,0,46,187,240]$ \\
\hline 18 & 0.7792 & 0.0033 & 0.0147 & 0.0042 & 0.0032 & $1,0,52,187,240]$ \\
\hline 19 & 0.7792 & 0.0033 & 0.0147 & 0.0042 & 0.0032 & $1,0,52,187,240]$ \\
\hline 20 & 0.7792 & 0.0033 & 0.0147 & 0.0042 & 0.0032 & $1,0,52,187,240]$ \\
\hline 21 & 0.6500 & 0.0496 & 0.1315 & 0.0708 & 0.0460 & {$[17,0,67,156,240]$} \\
\hline 22 & 0.6500 & 0.0464 & 0.1238 & 0.0667 & 0.0433 & {$[16,0,68,156,240]$} \\
\hline 23 & 0.6500 & 0.0527 & 0.1393 & 0.0750 & 0.0488 & {$[18,0,66,156,240]$} \\
\hline 24 & 0.6500 & 0.0591 & 0.1548 & 0.0833 & 0.0542 & {$[20,0,64,156,240]$} \\
\hline 25 & 0.6500 & 0.0433 & 0.1161 & 0.0625 & 0.0406 & {$[15,0,69,156,240]$} \\
\hline 26 & 0.6500 & 0.0433 & 0.1161 & 0.0625 & 0.0406 & {$[15,0,69,156,240]$} \\
\hline 27 & 0.6500 & 0.0110 & 0.0310 & 0.0167 & 0.0108 & {$[4,0,80,156,240]$} \\
\hline 28 & 0.6500 & 0.0082 & 0.0232 & 0.0125 & 0.0081 & $3,0,81,156,240]$ \\
\hline 29 & 0.6500 & 0.0138 & 0.0387 & 0.0208 & 0.0135 & $5,0,79,156,240]$ \\
\hline 30 & 0.6500 & 0.0027 & 0.0077 & 0.0042 & 0.0027 & $1,0,83,156,240]$ \\
\hline 31 & 0.6500 & 0.0167 & 0.0464 & 0.0250 & 0.0163 & $6,0,78,156,240]$ \\
\hline 32 & 0.6500 & 0.0283 & 0.0774 & 0.0417 & 0.0271 & {$[10,0,74,156,240]$} \\
\hline
\end{tabular}

${ }^{a}$ Medidas Relativas com Pesos wraccR $=$ wrnegrelR $=$ wrsensR $=$ wrspecR $=$ novR (Lavrac et al., 1999)

Tabela 7.6: Valores das Medidas Relativas, com e sem Peso, para as Regras de $\mathbf{h}_{1}$ 


\begin{tabular}{|c|c|c|c|c|c|c|}
\hline \hline \multirow{2}{*}{$\begin{array}{c}\text { \# da } \\
\text { Regra }\end{array}$} & \multicolumn{7}{|c|}{ Medidas Relativas } & \multirow{2}{*}{$\begin{array}{c}\text { Valores Absolutos } \\
{[}\end{array}$} & raccR & rnegrelR & rsensR & rspecR & wraccR $^{a}$ & {$[h \bar{h}, \overline{h b}, N]$} \\
\hline 1 & 0.4819 & 0.1112 & 0.2106 & 0.1583 & 0.0904 & {$[41,4,62,133,240]$} \\
\hline 2 & 0.5708 & 0.0248 & 0.0554 & 0.0417 & 0.0238 & {$[10,0,93,137,240]$} \\
\hline 3 & 0.5708 & 0.0222 & 0.0499 & 0.0375 & 0.0214 & {$[9,0,94,137,240]$} \\
\hline 4 & 0.4508 & 0.0524 & 0.1094 & 0.0823 & 0.0470 & {$[22,3,81,134,240]$} \\
\hline 5 & 0.4201 & 0.1837 & 0.2978 & 0.2239 & 0.1278 & {$[62,11,41,126,240]$} \\
\hline 6 & 0.5708 & 0.0097 & 0.0222 & 0.0167 & 0.0095 & {$[4,0,99,137,240]$} \\
\hline 7 & 0.4458 & 0.0154 & 0.0346 & 0.0260 & 0.0149 & {$[7,1,96,136,240]$} \\
\hline 8 & 0.3833 & 0.0274 & 0.0595 & 0.0448 & 0.0256 & {$[13,3,90,134,240]$} \\
\hline 9 & 0.7792 & 0.0200 & 0.0882 & 0.0250 & 0.0195 & {$[6,0,47,187,240]$} \\
\hline 10 & 0.7792 & 0.0166 & 0.0735 & 0.0208 & 0.0162 & {$[5,0,48,187,240]$} \\
\hline 11 & 0.6363 & 0.0191 & 0.0840 & 0.0238 & 0.0186 & {$[6,1,47,186,240]$} \\
\hline 12 & 0.7792 & 0.0033 & 0.0147 & 0.0042 & 0.0032 & {$[1,0,52,187,240]$} \\
\hline 13 & 0.5792 & 0.0123 & 0.0546 & 0.0155 & 0.0121 & {$[4,1,49,186,240]$} \\
\hline 14 & 0.5292 & 0.0090 & 0.0399 & 0.0113 & 0.0088 & {$[3,1,50,186,240]$} \\
\hline 15 & 0.5292 & 0.0090 & 0.0399 & 0.0113 & 0.0088 & {$[3,1,50,186,240]$} \\
\hline 16 & 0.7792 & 0.0065 & 0.0294 & 0.0083 & 0.0065 & {$[2,0,51,187,240]$} \\
\hline 17 & 0.6500 & 0.0527 & 0.1393 & 0.0750 & 0.0488 & {$[18,0,66,156,240]$} \\
\hline 18 & 0.5071 & 0.1076 & 0.2536 & 0.1365 & 0.0888 & {$[36,6,48,150,240]$} \\
\hline 19 & 0.4661 & 0.2650 & 0.4827 & 0.2599 & 0.1690 & {$[71,16,13,140,240]$} \\
\hline \hline
\end{tabular}

${ }^{a}$ Medidas Relativas com Pesos wraccR $=$ wrnegrel $\mathrm{R}=$ wrsensR $=$ wrspecR $=$ novR (Lavrac et al., 1999)

Tabela 7.7: Valores das Medidas Relativas, com e sem Peso, para as Regras de $\mathbf{h}_{2}$

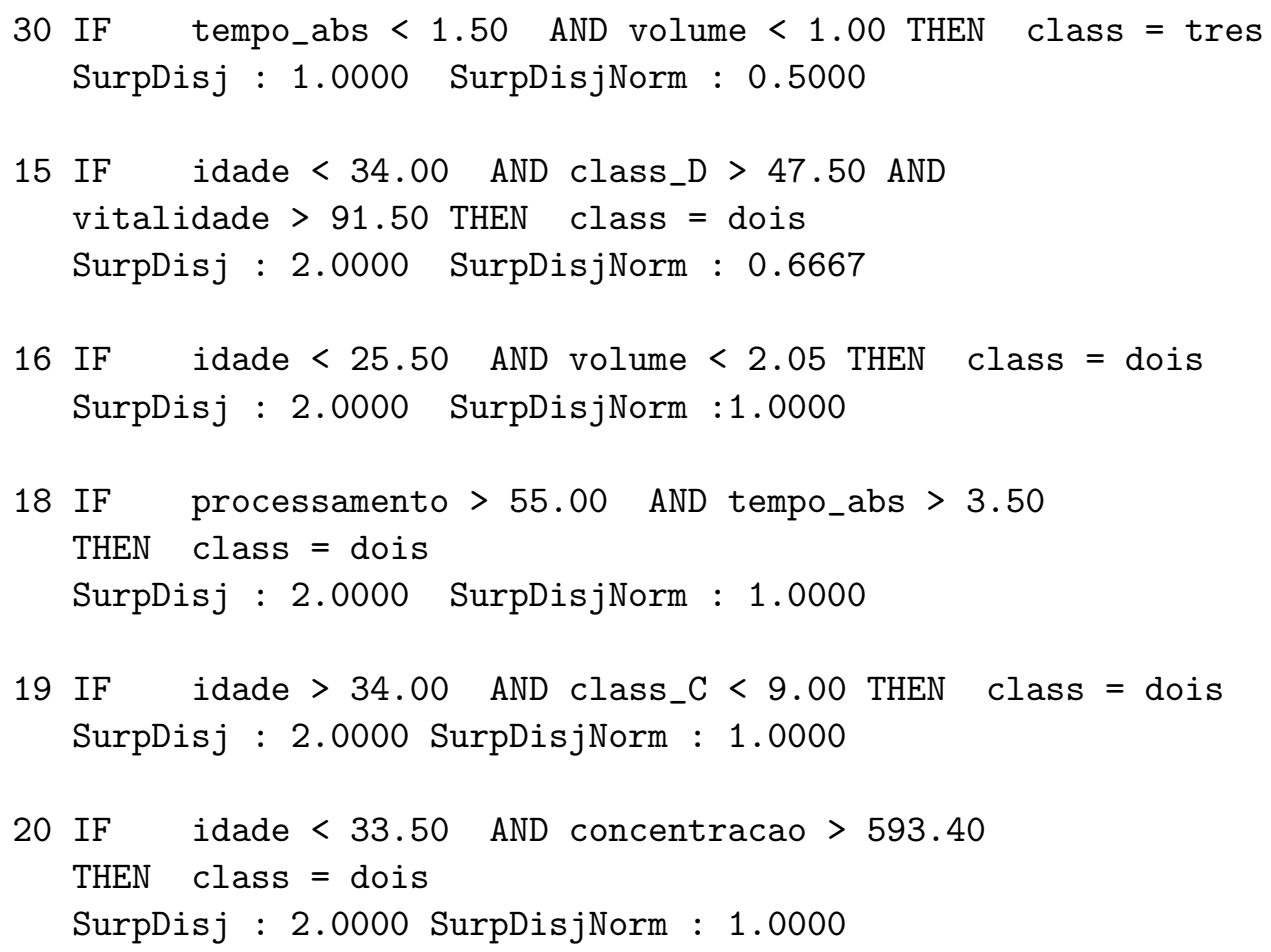

Figura 7.1: Surpresa dos Pequenos Disjuntos de $\mathbf{h}_{1}$ 


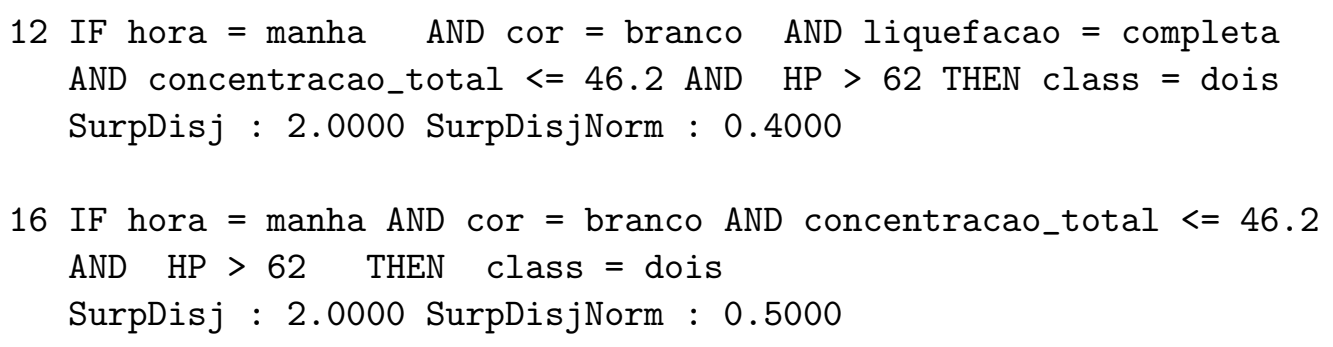

Figura 7.2: Surpresa dos Pequenos Disjuntos de $\mathbf{h}_{2}$

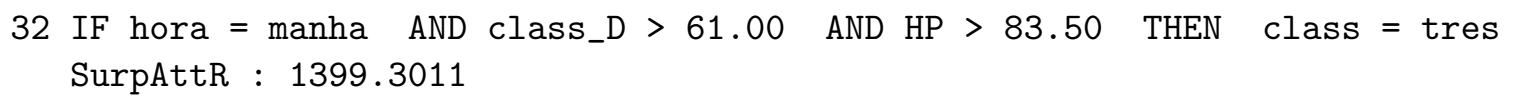

Figura 7.3: Surpresa dos Atributos Individuais Discretos das Regras Selecionadas de $\mathbf{h}_{1}$

\subsection{Discussão dos Resultados Obtidos}

Nesta seção é apresentada uma análise dos valores das medidas de avaliação de regras (análise qualitativa) bem como dos valores das medidas objetivas de interessabilidade de regras (análise quantitativa) obtidos nesse estudo de caso.

\subsubsection{Da Análise Qualitativa}

Conforme mencionado anteriormente, as "melhores" regras de um classificador simbólico h são aquelas que apresentam o maior (ou menor) valor para uma determinada medida de avaliação. Como essas medidas representam probabilidades, é importante considerar o número de exemplos utilizados para calcular essas probabilidades. Isto pode ser visto claramente no caso das 32 regras que constituem $\mathbf{h}_{1}$. Todas elas possuem o mesmo valor de accR $=1$ - Tabela 7.4.

Entretanto, a regra 1 cobre corretamente 32 exemplos, enquanto outras regras cobrem menos exemplos. Nesse caso, se a medida de accR é importante para o especialista, um outro critério válido seria considerar primeiro as regras com accR $=1$ que cobrem corretamente mais exemplos. A medida supR fornece essa informação. Como pode ser 
3 IF idade $>30$ AND idade $<=33$ AND cor $=$ branco AND concentracao_total $>95.2$ AND concentracao_total $<=338.8$ AND motilidade $<=15$ AND class_C $>18$ AND vitalidade $<=79$ AND HP $<=83$ THEN class $=u m$ SurpAttR: 24.4009

4 IF volume $>2.4$ AND cor $=$ branco AND concentracao_total $<=95.2$ AND class_A $<=33$ AND HP $<=88$ THEN class $=$ um

SurpAttR : 24.4009

7 IF idade $>35$ AND idade $<=36$ AND cor = branco AND motilidade $<=15$ THEN class $=$ um SurpAttR : 24.4009

9 IF volume $<=2.4$ AND cor $=$ branco AND concentracao_total $<=95.2$ AND motilidade $>17$ AND class_A $<=33$ AND HP $<=88$ THEN class $=$ dois SurpAttR : 24.4009

10 IF idade $>33$ AND idade $<=35$ AND volume $>2.1$ AND cor $=$ branco AND concentracao_total $>95.2$ AND concentracao_total $<=338.8$ AND motilidade $<=15$ AND class_C $>18$ AND HP $>56$ THEN class dois SurpAttR : 24.4009

16 IF hora = manha AND cor = branco AND concentracao_total <= 46.2 AND $\mathrm{HP}>62$ THEN class $=$ dois

SurpAttR : 47.9654

12 IF hora $=$ manha AND cor $=$ branco AND liquefacao $=$ completa AND concentracao_total <= 46.2 AND HP > 62 THEN class $=$ dois SurpAttR : 63.8402

Figura 7.4: Surpresa dos Atributos Individuais Discretos das Regras Selecionadas de $\mathbf{h}_{2}$ 
observado, são vários os critérios que podem ser utilizados pelo especialista para analisar as regras segundo essas medidas.

Para melhor visualizar a classificação das regras que constituem $\mathbf{h}_{1}$ e $\mathbf{h}_{2}$ segundo as diversas medidas apresentadas, respectivamente, nas Tabelas 7.4 e 7.6 para $\mathbf{h}_{1}$ e nas Tabelas 7.5 e 7.7 para $\mathbf{h}_{2}$, as regras de cada hipótese foram enumeradas e ordenadas de forma decrescente, segundo o "melhor" valor das medidas consideradas. Assim, na Tabela 7.8 são mostradas as regras de $\mathbf{h}_{1}$ ordenadas pelo "melhor" valor das medidas de avaliação (genéricas e relativas com e sem peso). Da mesma forma, nas Tabelas 7.9 e 7.10 são mostrados as regras de $\mathbf{h}_{2}$ ordenadas pelas medidas genéricas e relativas, respectivamente.

\begin{tabular}{|c|c|c|c|c|c|c|c|}
\hline \multirow{2}{*}{ Ordem } & \multicolumn{7}{|c|}{ Medidas de Avaliação } \\
\hline & negrelR & sensR & $\operatorname{covR}^{a}$ & novR $^{b}$ & rnegrelR & rsensR & rspecR \\
\hline 1 & 13 & 1 & 1 & 1 & 1 & 1 & 1 \\
\hline 2 & 17,12 & 6 & 6 & 6 & 6 & 6 & 6 \\
\hline 3 & 14 & 24 & 2 & 2 & 2 & 24 & 2 \\
\hline 4 & 16,15 & 2 & 4 & 4 & 4 & 23 & 4 \\
\hline 5 & $20,19,18$ & 4 & 24 & 24 & 24 & 2 & 24 \\
\hline 6 & 24 & 23 & 23,10 & 23 & 23 & 13 & 23,10 \\
\hline 7 & 23 & 21 & 21,3 & 21 & 21 & 21 & 21,3 \\
\hline 8 & 21 & 22 & 22,5 & 22 & 22 & 4 & 22,5 \\
\hline 9 & 22 & 26,25 & 26,25 & 10 & 10 & 22 & 26 \\
\hline 10 & 26,25 & 10 & 32 & 26,25 & 3 & 26,25 & 32 \\
\hline 11 & 32 & 13 & 13 & 3 & 26 & 17,12 & 13 \\
\hline 12 & 31 & 3 & 7 & 5 & 25 & 10 & 7 \\
\hline 13 & 29 & 5 & $17,12,8$ & 13 & 5 & 3 & $17,12,8$ \\
\hline 14 & 27 & 17 & 31 & 32 & 13 & 5 & 31 \\
\hline 15 & 1 & 12 & 29 & 17,12 & 32 & 32 & 29 \\
\hline 16 & 28 & 32 & 27,9 & 7 & 17,12 & 31 & 9,27 \\
\hline 17 & 30 & 7 & 28,14 & 8 & 7 & 7 & 28,14 \\
\hline 18 & 6 & 31 & 16,15 & 31 & 8 & 14 & 16,15 \\
\hline 19 & 2 & 8 & $30,20,19,18,11$ & 29 & 31 & 8 & $30,20,19,18,11$ \\
\hline 20 & 4 & 29 & & 27 & 29 & 29 & \\
\hline 21 & 10 & 14 & & 14 & 27 & 27 & \\
\hline 22 & 3 & 27 & & 28 & 14 & 16,15 & \\
\hline 23 & 5 & 9 & & 16,15 & 9 & 28 & \\
\hline 24 & 7 & 16,15 & & $20,19,18$ & 28 & 9 & \\
\hline 25 & 8 & 28 & & 30,11 & 16,15 & $20,19,18$ & \\
\hline 26 & 9 & $20,19,18$ & & & $20,19,18$ & 30 & \\
\hline 27 & 11 & 30 & & & 30 & 11 & \\
\hline 28 & & 11 & & & 11 & & \\
\hline
\end{tabular}

${ }^{a}$ covR $=\operatorname{supR}$.

${ }^{b}$ novR $=$ wraccR.

Tabela 7.8: Regras de $\mathbf{h}_{1}$ Ordenadas pelos Valores da Medida de Avaliação

\section{Considerando o Conjunto de Regras $\mathbf{h}_{1}$}

De um total de 14 diferentes medidas calculadas pelo MAR, sob o conjunto de regras $\mathbf{h}_{1}, 3$ dessas medidas (accR, specR e satR) apresentaram valor 1 para todas as regras. 
Assim, as 9 medidas de avaliação restantes foram sintetizadas nas Tabelas 7.4 e 7.6. Por sua vez, essas 9 medidas foram utilizadas para elencar as regras de $\mathbf{h}_{1}$ pela ordem decrescente dos valores calculados — Tabela 7.8. Deve ser observado que a medida errR é complementar à medida accR e por isso não foi considerada nessa tabela. Ainda deve ser observado que novR=wraccR e que, para esse conjunto de regras covR=supR.

Observando a Tabela 7.8 é possível verificar que as regras 1 e 6 apresentaram "melhor" valor para 6 (seis) das 9 (nove) medidas consideradas. Dessa forma, se o especialista considerar que a precisão na predição de exemplos não cobertos pela regra (ou seja, negrelR) não é um critério importante, pode-se dizer que as regras regras 1 e 6 são as que apresentam as melhores medidas de avaliação. Analogamente, as regras 2, 4, 24 e 23 são as próximas regras a apresentar boas medidas de avaliação. Isso dá uma indicação ao especialista para analisar mais cuidadosamente o subconjunto composto pelas regras $\{1,6,2,4,24$ e 23$\}$ - Figura 7.5.

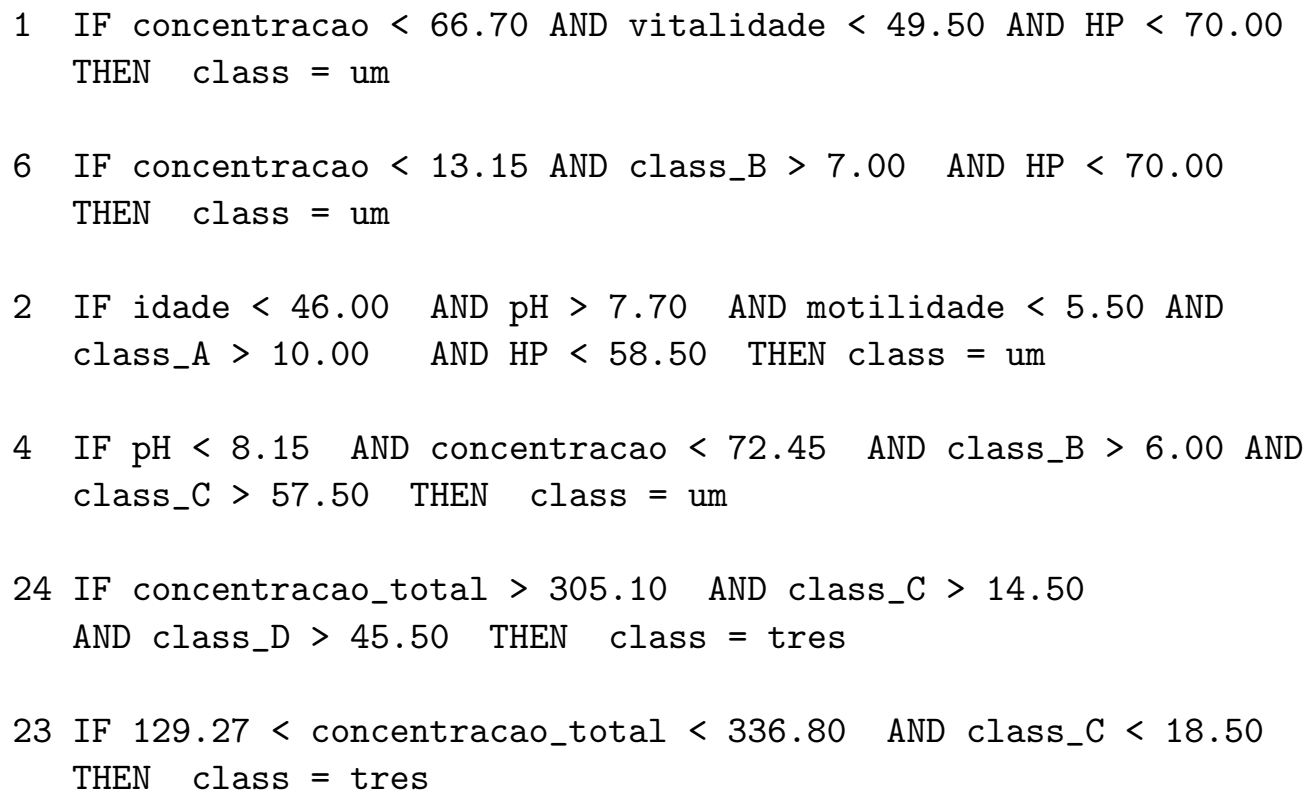

Figura 7.5: As "Melhores" Regras de $\mathbf{h}_{1}$

Inicialmente, um aspecto que chama a atenção ao ser observado, refere-se às classes preditas por cada regra do subconjunto das "melhores" regras de $\mathbf{h}_{1}$. Pode ser observado que as regras 1,2,4 e 6 predizem a classe um, enquanto que as regras 23 e 24 são da 
classe tres. Conseqüentemente, não se destacam regras que predizem a classe dois. É interessante observar que a classe dois é a classe minoritária do conjunto de exemplos utilizados, $22.08 \%$ - Tabela 7.1 - a classe um é a classe majoritária com $42.92 \%$ dos exemplos, enquanto que a classe tres possui $35.00 \%$ dos exemplos.

Outro aspecto que pode ser considerado refere-se aos atributos que compõem cada uma das regras desse subconjunto de $\mathbf{h}_{1}$. Observando novamente a Figura 7.5 é possível verificar que dos 21 atributos (exceto o atributo classe) do conjunto de dados utilizado apenas 10 estão presentes nos corpos das "melhores" regras de $\mathbf{h}_{1}$. Esses atributos são concentracao, vitalidade, hp, class B, idade, motilidade, class_A, class_C, class D e concentracao_total.

Finalmente, um outro aspecto que pode ser levado em conta refere-se a análise dos valores $h b, \bar{h} b$ de cada regra, ou seja, a análise dos valores referentes à cobertura da regra - Tabela 7.4, por exemplo. Como a precisão de todas as regras de $\mathbf{h}_{1}$ é igual a 1 , o valor $\bar{h} b$ é igual a 0 , ou seja, todas as regras de $\mathbf{h}_{1}$ não cobrem exemplos de uma classe diferente da predita pela regra. Considerando as regras $\{1,6,2,4,24$ e 23$\}$ é possível observar que o valor $h b$ da regra 1 é maior que o $h b$ da regra 6 , e assim por diante. Dessa forma, a regra 1 cobre corretamente mais exemplos que a regra 6 , que por sua vez, cobre mais exemplos que a regra 2 e assim segue.

\section{Considerando o Conjunto de Regras $\mathrm{h}_{2}$}

A partir das 14 diferentes medidas calculadas pelo MAR, apresentadas nas Tabelas 7.5 e 7.7, as regras $\mathbf{h}_{2}$ foram dispostas em ordem decrescente dos valores calculados para as medidas genéricas e relativas - Tabelas 7.9 e 7.10, respectivamente. Deve ser lembrado que a medida errR é complementar a accR e que novR = wraccR.

Dentre as 12 (doze) diferentes medidas consideradas, 7 (sete) apontam as regras 19 e 5 como sendo "melhores". As próximas melhores regras são 9,10,16 e 12, apontadas por 4 (quatro) medidas não consideradas entre as sete anteriores. Assim, o subconjunto das "melhores" regras de $\mathbf{h}_{2}$ é composto pelas regras $\{19,5,9,10,16$ e 12$\}$ — Figura 7.6.

Do subconjunto das "melhores" regras de $\mathbf{h}_{2}$, com relação às classes preditas por cada regra, a regra 5 prediz a classe um, as regras 9,10,12 e 16 predizem a classe dois, enquanto que a regra 19 prediz a classe tres.

Com relação aos atributos que compõem cada regra, pode ser observado que em todas 


\begin{tabular}{|c|c|c|c|c|c|c|c|}
\hline \multicolumn{8}{|c|}{ Medidas Genéricas } \\
\hline accR & negrelR & sensR & specR & covR & supR & novR ${ }^{a}$ & satR \\
\hline $17,16,12,10,9,6,3,2$ & 19 & 19 & $17,16,12,10,9,6,3,2$ & 19 & 19 & 19 & $17,16,12,10,9,6,3,2$ \\
\hline 1 & 9 & 5 & $15,14,13,11$ & 5 & 5 & 5 & 1 \\
\hline 4 & 11 & 18 & 7 & 1 & 1 & 1 & 11 \\
\hline 7 & 10 & 1 & 8,4 & 18 & 18 & 18 & 4 \\
\hline 18,11 & 13 & 17 & 1 & 4 & 4 & 17 & 7 \\
\hline 5 & 15,14 & 4 & 18 & 17 & 17 & 4 & 18 \\
\hline 19 & 16 & 8 & 5 & 8 & 8 & 8 & 13 \\
\hline 8 & 12 & 11,9 & 19 & 2 & 2 & 2 & 5 \\
\hline 13 & 18 & 2 & & 3 & 3 & 3 & 19 \\
\hline \multirow[t]{9}{*}{15,14} & 5 & 10 & & 7 & 7 & 9 & 15 \\
\hline & 17 & 3 & & 11 & 11,9 & 11 & 14 \\
\hline & 1 & 13 & & 9 & 10 & 10 & 8 \\
\hline & 4 & 7 & & 13,1 & 13,6 & 7 & \\
\hline & 8 & 15,14 & & $15,14,6$ & 15,14 & 13 & \\
\hline & 2 & 6 & & 16 & 16 & 6 & \\
\hline & 3 & 16 & & 12 & 12 & 15,14 & \\
\hline & 7 & 12 & & & & 16 & \\
\hline & 6 & & & & & 12 & \\
\hline
\end{tabular}

${ }^{a}$ novR $=$ wraccR.

Tabela 7.9: Regras de $\mathbf{h}_{2}$ Ordenadas pelos Valores das Medidas Genéricas

\begin{tabular}{|c|c|c|c|}
\hline \hline \multicolumn{4}{|c|}{ Medidas Relativas } \\
\hline raccR & rnegrelR & rsensR & rspecR \\
\hline $16,12,10,9$ & 19 & 19 & 19 \\
17 & 5 & 5 & 5 \\
11 & 1 & 18 & 1 \\
13 & 18 & 1 & 18 \\
$6,3,2$ & 17 & 17 & 4 \\
15,14 & 4 & 4 & 17 \\
18 & 8 & 9 & 8 \\
1 & 2 & 11 & 2 \\
19 & 3 & 10 & 3 \\
4 & 9 & 8 & 7 \\
7 & 11 & 2 & 9 \\
5 & 10 & 13 & 11 \\
8 & 7 & 3 & 10 \\
& 13 & 15,14 & 6 \\
& 6 & 7 & 13 \\
& 15,14 & 16 & 15,14 \\
& 16 & 6 & 16 \\
& 12 & 12 & 12 \\
\hline \hline
\end{tabular}

Tabela 7.10: Regras de $\mathbf{h}_{2}$ Ordenadas pelos Valores das Medidas Relativas. 


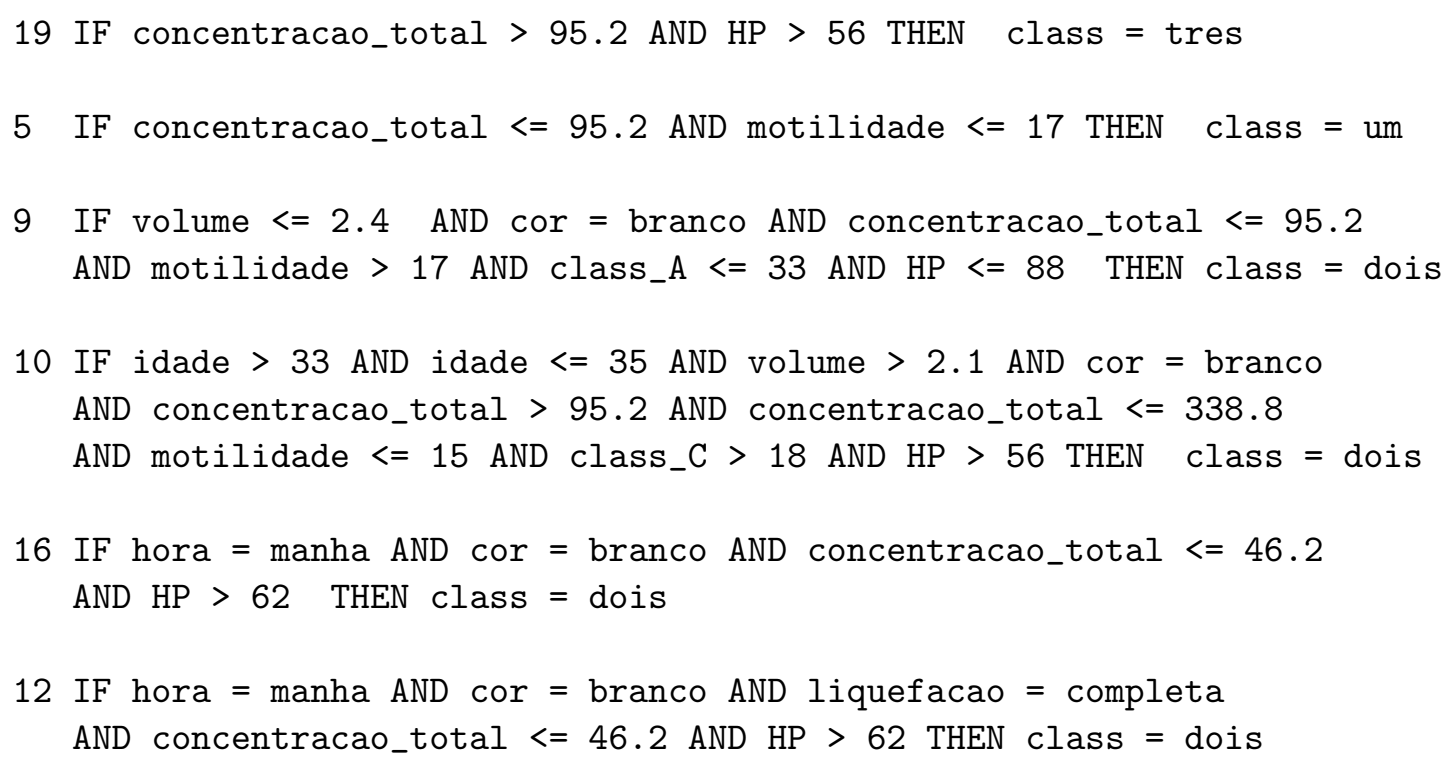

Figura 7.6: As "Melhores" Regras de $\mathbf{h}_{2}$

regras o atributo concentracao_total está presente. Dos 21 atributos (exceto o atributo classe) do conjunto de dados utilizado, novamente apenas 10 estão presentes nos corpos das "melhores" regras de $\mathbf{h}_{2}$. Esses atributos são concentracao_total, motilidade, hp, hora, cor, liquefação, idade, volume, class_A e class_C .

Considerando agora os valores $h b, \bar{h} b$, mostrados na Tabela 7.5 página 103 , de cada regra do subconjunto composto pelas "melhores" regras de $\mathbf{h}_{2}$ é possível observar que o valor $h b$ e $\bar{h} b$ da regra 19 é maior que os valores $h b$ e $\bar{h} b$ da regra 5 . É interessante observar por esses valores que a regra 19, mesmo cobrindo mais exemplos de classes diferentes da sua ( $\bar{h} b$ maior) foi apontada como "melhor" que a regra 5, i.e. apresentou "melhores" valores para as medidas de avaliação calculadas.

Com relação às regras restantes $\{9,10,16$ e 12$\}$, é possível observar que o valor $h b$ da regra 9 é maior que o $h b$ da regra 10 e assim por diante. É interessante observar que o valor de $h b$ dessas regras é muito menor se comparados com os valores de $h b$ das regras 19 e 5 mas, sob o ponto de vista da medida de precisão accR, as 9,10,16 e 12 são "melhores" que as regras 19 e 5. 


\subsubsection{Da Análise Quantitativa}

Num processo de análise quantitativa, quanto mais alto o valor de uma medida para uma determinada regra, maior é a chance de que o conhecimento descrito por ela seja um conhecimento interessante ou inesperado. Considerando os valores calculados pelo MAR, as regras mais interessantes de $\mathbf{h}_{1}$ e $\mathbf{h}_{2}$ são apresentadas na Tabela 7.11 na ordem decrescente dos valores calculados para cada medida objetiva de interessabilidade.

\begin{tabular}{|c|c|c|c|}
\hline \hline \multicolumn{2}{|c|}{$\mathbf{h}_{1}$} & \multicolumn{2}{c|}{$\mathbf{h}_{2}$} \\
\hline SurpDisj & SurpAttR & SurpDisj & SurpAttR \\
\hline $20,19,18,15$ & 32 & 12 & $3,4,7,9,10$ \\
16 & & 16 & 12 \\
30 & & & 16 \\
\hline \hline
\end{tabular}

Tabela 7.11: Regras de $\mathbf{h}_{1}$ e $\mathbf{h}_{2}$ Ordenadas pelos Valores da Medidas de Interessabilidade

\section{Considerando o Conjunto de Regras $\mathrm{h}_{1}$}

De um total de trinta e duas (32) regras de $\mathbf{h}_{1}$, foram selecionadas pelo MAR, através da medida de Surpresa de Pequenos Disjuntos, as regras 20, 19, 18, 15, 16 e 30 (Tabela 7.11). Pela medida de Surpresa dos Atributos Individuais Discretos foi selecionada apenas a regra 32 (Tabela 7.11 ).

Pode ser observado - Figuras 7.1 e 7.3 - que, desse subconjunto de regras apontadas como "mais interessantes", as regras 20,19,18,15 e 16 predizem a classe dois (classe minoritária), que as regras 30 e 32 predizem a classe três e, portanto, nenhuma dessas regras predizem a classe um (classe majoritária).

Dos 21 atributos (exceto o atributo classe) do conjunto de dados utilizado, apenas 11 estão presentes nos corpos das "mais interessantes" regras de $\mathbf{h}_{1}$. Esses atributos são hora, idade, concentracao, class_C, vitalidade, hp, volume, class D, processamento e tempo_abs. Outro aspecto a ser observado é que os corpos das regras são compostos por menos de 4 diferentes atributos.

Considerando agora os valores $h b, \bar{h} b$, mostrados na Tabela 7.4 página 103, é interessante observar que todas as regras tem $\bar{h} b=0$ e ainda, que dentre essas regras, a regra 32 chama atenção pois trata-se da única regra de $\mathbf{h}_{1}$ que possui em seu corpo atributos discretos e ainda, o número de exemplos corretamente cobertos $(h b)$ por essa regra é maior que as demais regras selecionadas como "mais interessantes". 


\section{Considerando o Conjunto de Regras $\mathbf{h}_{2}$}

Do conjunto de regras $\mathbf{h}_{2}$ com dezenove (19) regras, foram selecionadas através do cálculo da medida de Surpresa de Pequenos Disjuntos efetuado pelo MAR, as regras 12 e 16 (Tabela 7.11). Através da medida de Surpresa dos Atributos Individuais Discretos, foram selecionadas as regras 3,4,7,9,10,12 e 16 (Tabela 7.11).

Desse subconjunto de regras "mais interessantes" composto pelas regras $\{3,4,7,9,10,12$ e 16\}, pode ser observado — Figuras 7.2 e 7.4 — que as regras 3, 4 e 7 predizem a classe um (classe majoritária) e que as regras 9,10,12 e 16 predizem a classe dois e, portanto, nenhuma regra selecionada prediz a classe tres.

Pode ser facilmente constatado que as regras 12 e 16 foram selecionadas pelas duas medidas. Espera-se que essas regras tenham, potencialmente, mais interesse para o especialista do que as outras.

Dos 21 atributos (exceto o atributo classe) do conjunto de dados utilizado, novamente apenas 11 estão presentes nos corpos das "mais interessantes" regras de $\mathbf{h}_{2}$. Esses atributos são hora, idade, cor, concentracao_total, motilidade, class_c, vitalidade, hp, volume, class_a e liquefação. Deve ser observado que as regras desse subconjunto de $\mathbf{h}_{2}$ tem corpos compostos por mais de 4 atributos e ainda, que o atributo cor está presente em todas essas regras.

Finalmente, se forem comparados os subconjuntos de regras selecionadas pelas medidas qualitativas com o subconjunto de regras selecionadas pelas medidas quantitativas,considerando valores $h b, \bar{h} b$ de cada regra — por exemplo na Tabela 7.5 página 103 pode ser observado que as regras 9,10,12 e 16 estão presentes em ambos subconjuntos de regras de $\mathbf{h}_{2}$. Dessa forma, é curioso observar que essas quatro (4) regras cobrem poucos exemplos mas, ao mesmo tempo, cobrem corretamente esses exemplos. Ainda deve ser observado que as regras 12 e 16 apresentam o valor $h b$ menor que as outras regras selecionadas de $\mathbf{h}_{2}$.

\subsection{Considerações Finais}

No estudo apresentado neste trabalho, foi sucintamente descrita a importância do processamento de sêmen diagnóstico no tratamento para a reprodução assistida e também foi rapidamente descrito o Módulo de Análise de Regras do $\mathcal{R}_{\text {ule }} \mathcal{S}_{\text {ystem. }}$. Foram apresentadas 
as etapas envolvidas nesse estudo e ainda foram discutidos os resultados obtidos.

Vale ressaltar que inexiste uma metodologia estabelecida para a discussão de resultados obtidos através da utilização de medidas de avaliação e interessabilidade de regras. Neste trabalho optou-se por elencar as regras de acordo com o maior valor das medidas aqui consideradas e então observar quais regras que se destacam.

Os resultados foram discutidos levando em conta os aspectos considerados relevantes sob o ponto de vista da análise objetiva dos resultados. Na análise subjetiva, ou seja, a análise dos resultados feita por um especialista, podem ser utilizados, ou não, os resultados da análise objetiva. A idéia envolvida neste estudo de caso é que, mediante o uso das medidas de avaliação e de interessabilidade, o especialista possa focalizar sua atenção sob aquelas regras de maior destaque, evitando assim que esse especialista examine cada regra do conjunto de regras que constituem a(s) hipótese(s). Nesse contexto, a participação do especialista do domínio é fundamental que pode, inclusive discordar de alguns resultados. 
116 Capítulo 7: Estudo de Caso Utilizando o Módulo de Análise de Regras do $\mathcal{R}_{\text {ule }} \mathcal{S}_{\text {ystem }}$ 


\section{Capítulo 8}

\section{Conclusão}

As pesquisas na área de Aprendizado de Máquina tem contribuído de forma bastante significativa para o desenvolvimento de tecnologias voltadas para Data Mining. Uma dessas contribuições significativas, abordada neste trabalho, refere-se a utilização de algoritmos de AM na extração de conhecimento em grandes bases de dados.

Inicialmente, no Capítulo 2 deste trabalho, foram apresentadas algumas características gerais da área de Aprendizado de Máquina, enfocando os sistemas de AM capazes de induzir conhecimento (classificador) a partir de um conjunto de dados. Quando esse conhecimento está descrito de forma inteligível ao ser humano, ele pode ser transformado em conjuntos de regras if-then, ou seja, regras do tipo Corpo $\rightarrow$ Cabeça ou Body $\rightarrow$ Head.

Descrito sob a forma de regras, o conhecimento extraído pode ser analisado de diferentes maneiras por parte do usuário. No entanto, se o volume de regras for muito grande, a análise manual desse conhecimento simbólico torna-se difícil e muito custosa. Para sanar essa dificuldade, a análise automática de regras, sob os aspectos qualitativo e quantitativo, torna-se um recurso poderoso que pode ser utilizado.

Sob o aspecto qualitativo, regras podem ser analisadas com o intuito de saber quais são aquelas melhor sustentadas pelos dados. A análise qualitativa pode ser efetuada utilizando medidas de avaliação de regras como as apresentadas no Capítulo 3. Sob o aspecto quantitativo, regras podem ser analisadas com o intuito de selecionar aquelas regras que descrevem um conhecimento interessante ou inesperado para o usuário. Para efetuar a análise quantitativa de regras podem ser utilizadas as medidas de interessabilidade apresentadas no Capítulo 4. 
No intuito de oferecer funcionalidades voltadas para AM e DM, como por exemplo a análise automática de regras, foi implementado o $\mathcal{R}_{\text {ule }} \mathcal{S}_{\text {ystem }}$, apresentado neste trabalho no Capítulo 5, utilizando a linguagem de programação lógica Prolog.

A linguagem lógica Prolog permitiu a rápida prototipagem dos módulos que compõem o $\mathcal{R}_{\text {ule }} \mathcal{S}_{\text {ystem. }}$ O ambiente de programação LPA Prolog (Westwood, 2000b; Westwood, 2000a), utilizado no desenvolvimento desse sistema, já incorpora uma série de comodidades para a implementação de ferramentas computacionais, facilitando a familiarização ao paradigma de programação lógica e também a detecção de erros de programação.

A sintaxe padrão Prolog, proposta para estabelecer o formato dos dados de entrada do $\mathcal{R}_{\text {ule }} \mathcal{S}_{\text {ystem }}$, pretende ser robusta o suficiente para ser utilizada em outras aplicações implementadas nessa linguagem de programação lógica. Da mesma forma, a metodologia PEFD, proposta para a documentação do sistema, pode ser utilizada e melhorada para que outras aplicações possam ser formalmente especificadas através dessa metodologia.

Como mencionado anteriormente, o processo de descoberta de conhecimento em bases de dados é um processo não-trivial que envolve várias etapas, dentre elas a etapa de Data Mining, na qual o conhecimento é extraído e pode estar descrito sob a forma de regras. Por tratar-se de um processo de grandes proporções, sua modelagem computacional apresenta uma complexidade de elaboração também grandiosa, emergindo assim a necessidade de que algumas funcionalidades sejam implementadas e testadas antes de serem efetivamente incorporadas a um modelo computacional desse grande processo. No intuito de oferecer um ambiente integrado para apoiar as etapas do processo de descoberta de conhecimento, está em desenvolvimento no LABIC/ICMC o projeto Discover, cujo porte e complexidade de modelagem requer que algumas idéias sejam testadas antes de serem efetivamente incorporadas a esse ambiente.

Dessa forma, as funcionalidades voltadas para AM e DM implementadas no $\mathcal{R}_{\text {ule }} \mathcal{S}_{\text {ystem }}$ , tornam esse sistema computacional protótipo extremamente útil para testar algumas idéias que poderão, futuramente, ser incorporadas ao ambiente Discover. Por exemplo, a análise automática de regras de conhecimento pode ser realizada fazendo uso dos procedimentos implementados no Módulo de Análise de Regras do $\mathcal{R}_{\text {ule }} \mathcal{S}_{\text {ystem }}$, descrito neste trabalho no Capítulo 6.

Por meio do estudo de caso apresentado no Capítulo 7 é possível verificar o comportamento do $\mathcal{R}_{\text {ule }} \mathcal{S}_{\text {ystem }}$ no tratamento de problemas do mundo real. Apesar de inexistir uma 
metodologia estabelecida para a discussão de resultados obtidos através da utilização de medidas de avaliação e interessabilidade de regras, implementadas no MAR, neste trabalho optou-se por elencar as regras de acordo com o maior valor dessas medidas e então observar quais são as regras que se destacam.

Essa metodologia empregada na análise dos resultados obtidos no estudo de caso, pode ser vista como uma análise objetiva do conhecimento extraído. Na análise subjetiva, ou seja, na análise do conhecimento extraído feita por um especialista, podem ser utilizados ou não os resultados apontados pela análise objetiva. Dessa forma, mediante o uso das medidas de avaliação e de interessabilidade, o especialista pode realmente focalizar sua atenção sob aquelas regras que mais se destacam, evitando assim, que esse especialista examine uma a uma cada regra do conjunto de regras que constituem a(s) hipótese(s). Nesse contexto, a participação do especialista do domínio é fundamental que pode, inclusive discordar de alguns resultados apresentados.

Um dos aspectos positivos do $\mathcal{R}_{\text {ule }} \mathcal{S}_{\text {ystem }}$ é que, por ter sido implementado na linguagem de programação Prolog, após realizado os cálculos das melhores medidas implementadas no MAR, o usuário do sistema pode realizar quaisquer tipo de interrogações on-line tais como:

Quais as regras que possuem máximo valor de accR e cobrem pelo menos $X \%$ do número de exemplos?

Quais as regras com máximo valor para as medidas covR e novR?

Quais as regras comuns selecionadas pelas medidas SurpDisjR e SurpAtrR?

A partir das idéias implementadas no $\mathcal{R}_{\text {ule }} \mathcal{S}_{\text {ystem }}$ vários trabalhos futuros poderão ser realizados. Dentre eles, o mais imediato é o projeto e a implementação de uma interface para esse sistema. A metodologia empregada na elaboração dessa interface pode, por exemplo, servir de modelo para a implementação da interface do projeto Discover. Um outro trabalho, que pode ser realizado facilmente, é a incorporação de novas medidas de qualidade, desde que também unificadas sob a perspectiva probabilística do framework de Lavrac, e ainda a incorporação de novas medidas objetivas de interessabilidade. A incorporação de medidas subjetivas de interessabilidade deve ser cuidadosamente estudada, já que essas medidas possuem a característica de serem dependentes em relação ao domínio de aplicação. 
Também aproveitando-se da característica de prototipagem rápida oferecida pela linguagem Prolog, outros módulos podem ser facilmente incorporados ao $\mathcal{R}_{\text {ule }} \mathcal{S}_{\text {ystem }}$, inclusive explorando características que não estejam pré-definidas no sistema, ou ainda, aproveitar procedimentos específicos, implementados em cada módulo do $\mathcal{R}_{\text {ule }} \mathcal{S}_{\text {ystem }}$, para que novas funcionalidades sejam futuramente implementadas e testadas, antes de serem efetivamente incorporadas ao projeto Discover. 


\section{Referências}

Aha, D., Kibler, D., and Albert, M. K. (1991). Instance-based learning algorithms. Machine Learning, 1(6):37-66.

Baranauskas, J. A. (2001). Extração automática de conhecimento por múltiplos indutores. Tese de Doutorado, ICMC-USP. http://www.teses.usp.br/teses/disponiveis/55/55134/tde-08102001-112806/.

Baranauskas, J. A. and Batista, G. E. A. P. A. (2000). O projeto Discover: Idéias iniciais. (Comunicação Pessoal).

Baranauskas, J. A. and Monard, M. C. (2000). Reviewing some machine learning concepts and methods. Technical Report 102, ICMC-USP. ftp://ftp.icmc.sc.usp.br/pub/BIBLIOTECA/rel_tec/Rt_102.ps.zip.

Batista, G. E. A. P. A. (1997). Um ambiente de avaliação de algoritmos de aprendizado de máquina utilizando exemplos. Dissertação de Mestrado, ICMC-USP.

Batista, G. E. A. P. A. (2000). Pré-processamento de dados em aprendizado de máquina supervisionado. Monografia do Exame de Qualificação de Doutorado, ICMC-USP.

Batista, G. E. A. P. A. (2001). Sintaxe padrão do arquivo de exemplos do projeto DisCOVER. http://www.icmc.sc.usp.br/ gbatista/SintaxePadraoFinal.htm.

Bernardini, F. C. (2001). Combinação de classificadores para melhorar o poder preditivo e descritivo de ensembles. Monografia do Exame de Qualificação de Mestrado, ICMC-USP.

Bernardini, F. C. (2002). Combinação de classificadores para melhorar o poder preditivo e descritivo de ensembles. Dissertação de Mestrado, ICMC-USP (Em Elaboração).

Bernardini, F. C. and Monard, M. C. (2002a). Um estudo de caso utilizando o 
módulo de explicação e combinação do $\mathcal{R}_{\text {ule }} \mathcal{S}_{\text {ystem }}$. Technical Report 160, ICMCUSP. ftp://ftp.icmc.sc.usp.br/pub/BIBLIOTECA/rel_tec/Rt_160.ps.zip.

Bernardini, F. C. and Monard, M. C. (2002b). Um módulo de explicação de ensembles e combinação de regras. Technical Report 159, ICMC-USP. ftp://ftp.icmc.sc.usp.br/pub/BIBLIOTECA/rel_tec/Rt_159.ps.zip.

Braga, A., Carvalho, A., and Ludermir, T. (2000). Redes Neurais Artificiais: Teoria e Aplicações. LTC Press, Rio de Janeiro, Brasil.

Bratko, I. (1990). Prolog Programming for Artificial Intelligence. Addison-Wesley.

Breiman, L., Friedman, J., Olshen, R., and Stone, C. (1984). Classification and Regression Trees. Morgan Kaufmann Publishers, Wadsworth, CA.

Caulkins, C. W. (2000). Aquisição de conhecimento utilizando aprendizado de máquina relacional. Dissertação de Mestrado, ICMC-USP.

Clark, P. and Niblett, T. (1989). The $\mathcal{C N} 2$ induction algorithm. Machine Learning, $3(4): 261-283$.

Dean, P. and Famili, A. (1997). Comparative performance of rule quality measures in a induction system. Applied Intelligence, 7:113-124.

Esteves, S. and Bento, F. C. (1998). Sperm processing for assisted reproductive technology (art): effects of pentoxifyline on recovery of motile sperm in asthenozoospermic men. In FertilSteril, page 5196.

Esteves, S., Lee, H., Monard, M., and Lopes, L. (2001). Inteligência artificial aplicada à andrologia: um estudo de caso do processamento de sêmen diagnóstico. In XXVIII Congresso Brasileiro de Urologia, Fortaleza, CE, Brasil.

Esteves, S. C., Sharma, R. K., Thomas, A. J. J., and Agarwal, A. (2000). Improvement in motion characteristics and acrosome status in cryopreserved human spermatozoa by swim-up processing before freezing. In HumReprod, pages 2173-2179.

Fayyad, U. M., Piatetsky-Shapiro, G., and Smyth, P. (1996). From Data Mining to Knowledge Discovery: An Overview, pages 1-34. American Association for Artificial Intelligence. 
Félix, L. C. M. (1998). Data mining no processo de extração de conhecimento de bases de dados. Dissertação de Mestrado, ICMC-USP.

Félix, M., Rezende, S., Doi, C., de Paula, M., and Romanato, M. (1998). MLC $C^{++}$ biblioteca de aprendizado de máquina em $C^{++}$. Technical Report 72, ICMC-USP. ftp://ftp.icmc.sc.usp.br/pub/BIBLIOTECA/rel_tec/Rt_172.ps.zip.

Ferro, M., Lee, H. D., and Esteves, S. C. (2002). Intelligent data analysis: A case study of the diagnostic sperm processing. In International Conference on Computer Science, Software Engineering, Information Technology, e-Business, and Applications. (CSITeA-2002), (submetido).

Freitas, A. and Kirner, C. (1992). Introdução a algoritmos genéticos. In Anais do I Workshop sobre Redes Neurais, pages 71-88. UFSCar, São Carlos.

Freitas, A. A. (1998a). A multi-criteria approach for the evaluation of rule interestingness. In Proceedings of the International Conference on Data Mining, pages 7-20, Rio de Janeiro, RJ.

Freitas, A. A. (1998b). On objective measures of rule surprisingness. In Principles of Data Mining \& Knowledge Discovery: Proceedings of the Second European Symp. Lecture Notes in Artificial Intelligence, volume 1510, pages 1-9.

Goldberg, D. E. (1989). Genetic Algoritms in Search, Optimization an Machine Learning. Addison Wesley, Canadá.

Gomes, A. K. (2001). Medidas de avaliação de regras. Exame de Qualificação de Mestrado, ICMC-USP.

Gomes, A. K., Bernardini, F. C., and Monard, M. C. (2002). Uma sintaxe padrão Prolog para classificadores simbólicos. Technical Report 154, ICMC-USP. ftp://ftp.icmc.sc.usp.br/pub/BIBLIOTECA/rel_tec/Rt_154.ps.zip.

Gomes, A. K. and Monard, M. C. (2002a). Descrição do módulo de análise de regras do $\mathcal{R}_{\text {ule }} \mathcal{S}_{\text {ystem. }}$ Technical Report 155, ICMC-USP. ftp://ftp.icmc.sc.usp.br/pub/BIBLIOTECA/rel_tec/Rt_155.ps.zip.

Gomes, A. K. and Monard, M. C. (2002b). Um estudo de caso utilizando o módulo de análise de regras $\mathcal{R}_{\text {ule }} \mathcal{S}_{\text {ystem }}$. Technical Report 157, ICMC-USP. ftp://ftp.icmc.sc.usp.br/pub/BIBLIOTECA/rel_tec/Rt_158.ps.zip. 
Hilderman, R. J. and Hamilton, H. J. (1999). Knowledge discovery and interestingness measures: A survey. Technical Report s4s, University of Regina.

Holland, J. H. (1986). Escaping brittleness: The possibilities of general-purpose learning algorithms applied to parallel rule-based systems. Machine Learning: An Artificial Intelligence Approach, 2:27.

Hopfield, J. J. (1982). Neural networks and physical systems with emergent collective computational abilities. In International Academy of Science of the U.S.A., volume 81, pages 3088-3092.

Horst, P. and Monard, M. (2000). Um sistema computacional para avaliaç ao de regras induzidas por algoritmos de aprendizado de máquina. In Proceedings IBERAMIASBIA 2000 Open Discussion Track, pages 167-176, Atibaia, SP, Brasil.

Horst, P. S. (1999). Avaliação do conhecimento adquirido por algoritmos de aprendizado de máquina utilizando exemplos. Dissertação de Mestrado, ICMC-USP.

Imamura, C. Y. (2001). Pré-processamento para extração de conhecimento de bases textuais. Dissertação de Mestrado, ICMC-USP.

Lavrac, N. and Dzeroski, S. (1994). Inductive Logic Programming: Techniques and Applications. Ellis Horwood, London.

Lavrac, N., Flach, P., and Zupan, B. (1999). Rule evaluation measures: a unifying view. In Proceedings of the Ninth International Workshop on Inductive Logic Programming. LNAI, volume 1634, pages 74-185.

Lee, H. D. (2000). Seleção e construção de features relevantes para o aprendizado de máquina. Dissertação de Mestrado, ICMC-USP. http://www.teses.usp.br/teses/disponiveis/55/55134/tde-15032002-113112/.

Lee, H. D. and Monard, M. C. (2000a). Indução construtiva guiada pelo conhecimento: Um estudo de caso do processamento de smen diagnóstico. In Proceedings IBERAMIA-SBIA 2000, Open Discussion Track, pages 167-176, Atibaia, SP, Brasil.

Lee, H. D. and Monard, M. C. (2000b). A practical approach for knowledge-driven constructive induction. In Procedings Argentine Symposium on Artificial Intelligence, ASAI'2000, 29th International Conference SADIO, pages 71-86, Argentina. 
Major, J. A. and Mangano, J. J. (1993). Selecting among rules induced from a hurricane database. In Press, A., editor, Knowledge Discovery in Databases Workshop, pages 28-44, Melo Park, Califórnia.

Martins, C. A. (2001). Interpretação de clusters em aprendizado de máquina. Exame de Qualificação de Doutorado, ICMC-USP.

McCulloch, W. S. and Pitts, W. (1943). A logical calculus of the ideas immenet in nervous activity. In Boulletin of Mathematical Biophisics, volume 5, pages 115-133.

Michalski, R., Carbonel, J. G., and Mitchell, T. M. (1983). Machine Learning - An Artificial Intelligence Aproach. Tioga Publising Company, Palo Alto, California.

Michalski, R. S., Mozetic, I., Hong, J., and Lavrac, N. (1986). The multi-purpose incremental learning system AQ15 and its testing application to three medical domains. In Proceedings of the Fifth Annual National Conference on Artificial Intelligence, volume 1510, pages 1041-1045.

Milaré, C. R. (2000). Extração de conhecimento de redes neurais. Exame de Qualificação de Doutorado, ICMC-USP.

Minsky, M. L.; Papert, S. (1969). Perceptrons. MIT Press, Cambridge.

Mitchell, T. (1997). Machine Learning. McGraw Hill.

Monard, M. C., Batista, G. E. A. P. A., Kawamoto, S., and Pugliesi, J. B. (1997). Uma introdução ao aprendizado simbólico de máquina. Notas Didáticas, 29. Instituto de Matemática e Computação. Universidade de São Paulo. ftp://labic.icmc.sc.usp.br/didatico/PostScript/ML.ps.

Morgan, J. and Messenger, R. (1973). Thaid: A sequencial search program for the analysis of nominal scale dependent variables. Technical report, Institute for Social Research, University of Michigan. Technical Report.

Murthy, S. K., Kasif, S., and Salzberg, S. L. (1994). A system for induction of oblique decision trees. Journal of Artificial Intelligence Research, 2(1):1-32. http://www .

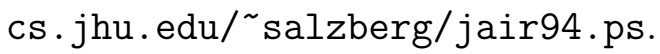

Nagai, W. A. (2000). Avaliação do conhecimentos extraído de problemas de regressão. Dissertação de Mestrado, ICMC-USP. 
Piatetsky-Shapiro, G. (1991). Discovery, analysis and presentation of strong rules. In Piatetsky-Shapiro, G. and Frawley, W. J., editors, Knowledge Discovery in Databases, pages 229-248.

Pila, A. D. (2000). Seleção de atributos relevantes para aprrendizado de máquina utilizando a abordagem de rough sets. Dissertação de Mestrado, ICMCUSP. http://www.teses.usp.br/teses/disponiveis/55/55134/tde-13022002-153921/publico/dissertacao_ADP.pdf.

Pistory, E. (1998). Construção automática de teoria de grafos. Dissertação de Mestrado, IC-UNICAMP.

Prati, R. C., Baranauskas, J. A., and Monard, M. C. (2001a). Extração de informações padronizadas para a avaliação de regras induzidas por algoritmos de aprendizado de máquina simbólico. Technical Report 145, ICMC-USP. ftp://ftp.icmc.sc.usp.

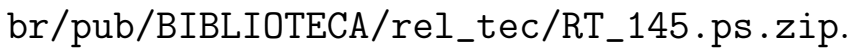

Prati, R. C., Baranauskas, J. A., and Monard, M. C. (2001b). Uma proposta de unificação da linguagem de representação de conceitos de algoritmos de aprendizado de máquina simbólicos. Technical Report 137, ICMC-USP. ftp://ftp.icmc.sc.usp. br/pub/BIBLIOTECA/rel_tec/RT_137.ps.zip.

Provost, F. J. and Aronis, J. M. (1996). Scaling up inductive learning with massive parallelism. Machine Learning, 23(1):33-46.

Pugliesi, J. B. (2001). O pós-processamento em extração de conhecimento de bases de dados. Exame de Qualificação de Doutorado, ICMC-USP.

Quinlan, J. R. (1986). Induction of decision trees. Machine Learning, 1:81-106. Reprinted in Shavlik and Dietterich (eds.), 1990. Readings in Machine Learning, Morgan Kaufmann Publishers, Inc.

Quinlan, J. R. (1987). Generating production rules from decision trees. In Proceedings of the Tenth International Joint Conference on Artificial Intelligence, pages 304-307, Italy.

Quinlan, J. R. (1993). C4.5: Programs for Machine Learning. Morgan Kaufmann Publishers, Los Altos, California, USA. 
Rosemblatt, F. (1985). The perceptron: A probabilistic model for information storage and organization in the brain. In Psycological Review, volume 65, pages 386-408.

Rumelhart, D. E. and McClelland, J. L. (1986). Parallel Distributed Processing: Explorations in the Microstructure of Cognition. MIT Press.

Russell, S. and Norvig, P. (1995). Artificial Intelligence: A Modern Approach. PrenticeHall.

Silberschatz, A. and Tuzhilin, A. (1995). On subjective measures of interestingness in knowledge discovery. In Proceedings of the First International Conference on Knowledge Discovery and Data Mining - KDD’95, pages 275-281. Montreal, Canadá.

Silberschatz, A. and Tuzhilin, A. (1996). What makes patterns interesting in knowledge discovery systems. IEEE Trans. on Knowledge and Data Eng., 8(6):970-974.

Stanfill, C. and Waltz, D. (1986). Instance-based learning algorithms. Communications of the ACM, 12:1213-1228.

Suzuki, E. and Kodratoff, Y. (1998). Discovery of surprising exception rules based on intensity of implication. In Principles of Data Mining \& Knowledge Discovery: Proceedings of the Second European Symp. Lecture Notes in Artificial Intelligence, volume 1510, pages 10-18.

Utgoff, P. F. (1989). Incremental induction of decision trees. Machine Learning, 4:161186.

Westwood, D. (2000a). LPA-PROLOG for windows programming guide 3.6, $3^{a}$ ed. http://www.lpa.co.uk.

Westwood, D. (2000b). LPA-PROLOG technical reference (programmerś reference) 3.0, $3^{a}$ ed. http://www.lpa.co.uk. 